Linköping Studies in Science and Technology,

Dissertation No. 1684

\title{
Interfacing nanomaterials for bioelectronic applications
}

\author{
Onur Parlak
}

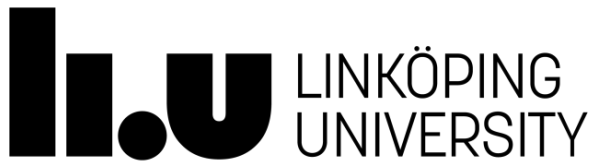

Biosensors and Bioelectronics Centre

Department of Physics, Chemistry, and Biology (IFM)

Linköping University, SE-581 83 Linköping, Sweden

Linköping 2015 


\section{Interfacing nanomaterials for bioelectronic applications}

\section{Onur Parlak}

During the course of the research underlying this thesis, Onur Parlak was enrolled in Forum Scientium, a multidisciplinary doctoral programme at Linköping University, Sweden.

Linköping studies in science and technology. Dissertation No. 1684

Copyright (c) Onur Parlak, 2015, unless otherwise noted

Cover Design by Onur Parlak

Printed in Sweden by LiU-Tryck, Linköping, Sweden, 2015

Published articles have been reprinted with the permission of the copyright holder.

ISBN 978-91-7519-028-0

ISSN 0345-7524 


\section{ABSTRACT}

The integration of nanomaterials as a bridge between the biological and electronic worlds has revolutionised understanding of how to generate functional bioelectronic devices and has opened up new horizons for the future of bioelectronics. The use of nanomaterials as a versatile interface in the area of bioelectronics offers many practical solutions and has recently emerged as a highly promising route to overcome technical challenges in the control and regulation of communication between biological and electronics systems. Hence, the interfacing of nanomaterials is yielding a broad platform of functional units for bioelectronic interfaces and is beginning to have significant impact on many fields within the life sciences.

In parallel with advancements in the successful combination of the fields of biology and electronics using nanotechnology in a conventional way, a new branch of switchable bioelectronics, based on signal-responsive materials and related interfaces, has begun to emerge. Switchable bioelectronics consists of functional interfaces equipped with molecular cues that are able to mimic and adapt to their natural environment and change physical and chemical properties on demand. These switchable interfaces are essential tools to develop a range of technologies to understand the function and properties of biological systems such as bio-catalysis, control of ion transfer and molecular recognition used in bioelectronics systems.

This thesis focuses on both the integration of functional nanomaterials to improve electrical interfacing between biological system and electronics and also the generation of a dynamic interface having the ability to respond to real-life physical and chemical changes. The development of such a dynamic interface facilitates studies of how living systems probe and respond to their changing environment and also helps to control and modulate bio-molecular interactions in a confined space using external physical and chemical stimuli. First, the integration of various nanomaterials is described, in order to understand the effect of different surface modifications and morphologies of various materials on enzymebased electrochemical sensing of biological analytes. Then, various switchable interfaces, based on graphene-enzyme and responsive polymer that could be modulated by temperature, light and $\mathrm{pH}$, were developed to control and regulate enzyme-based biomolecular reactions. Finally, a physically controlled programmable bio-interface is described by "AND" and "OR" Boolean logic operations using two different stimuli on one electrode. Together, the findings presented in this thesis contribute for the establishment of switchable and programmable bioe- 
lectronics. Both approaches are promising candidates to provide key building blocks for future practical systems, as well as model systems for fundamental research. 


\section{POPULARVETENSKAPLIG SAMMANFATTNINGS}

\section{Gränssnitt med nanomaterial för tillämpningar inom bioelektronik}

Gränssnittet mellan de biologiska och elektroniska världarna har gett upphov till en ny vetenskapsgren för att besvara mångåriga grundläggande frågor om levande system. Upptäckter som utgör milstolpar under det senaste århundradet inom både bioteknik och elektronik har underlättat konstruktionen av biogränssnitt och visat att elektronik kan integreras med bioteknik för att bygga nya sensorer, driva syntetiska reaktioner och alstra energi. Dessa framsteg har öppnat upp en ny era för "bioelektronik", som hjälper oss att förstå grunderna till olika biokemiska och/eller biofysikaliska händelser i levande system och bygga funktionella bioenheter. Det enorma bidrag som framsteg inom elektroniska komponenter har gjort till utvecklingen inom biokemi och bioteknik får inte heller underskattas. Detta har uppnåtts genom utformning och utveckling av nya metoder eller konstruktion av nya nanomaterial med olika funktionaliteter för att höja nivån inom bioelektronik.

I den här studien lägger vi fokus på det framväxande fältet för omkopplingsbara gränssnitt och deras följder för tillämpningar inom bioelektronik och energi. Vi försöker pussla ihop tidiga genombrott och de viktigaste stegen i utvecklingen, belysa och diskutera framtiden för omkopplingsbar bioelektronik genom att koncentrera oss på de senaste studierna för att förstå de kemiska och fysikaliska mekanismerna i levande system och hitta rimliga förklaringar till bioelektrokemiska processer baserat på imitation och kontroll av biologiska miljöer. 


\section{LIST OF INCLUDED PAPERS}

\section{Paper I}

Template-Directed Hierarchical Self-Assembly of Graphene Based Hybrid Structure for Electrochemical Biosensing

Onur Parlak, Atul Tiwari, Anthony P. F. Turner, Ashutosh Tiwari

Biosensors and Bioelectronics, 2013, 49, 53

Contribution: Designed whole work and performed all experimental work except graphene synthesis and characterisation. Wrote whole paper and contributed to the final editing of the manuscript.

\section{Paper II}

Two-dimensional Gold-Tungsten Disulphide Bio-interface for Highthroughput Electrocatalytic Nanobi oreactors

Onur Parlak, Prethi Seshadri, Ingemar Lundström, Anthony P. F. Turner Ashutosh Tiwari Advanced Materials Interfaces, 2014, 1, 1400136

Contribution: Designed whole work and performed all experimental work together with second author. Wrote whole paper and contributed to the final editing of the manuscript.

\section{Paper III}

Probing Ultra-Lightweight Aerographite Properties for Efficient Bioelectrocatalysis

Onur Parlak, Yogendra K. Mishra, Anton Grigoriev, Matthias Mecklenburg, Karl Schulte, Rajeev Ahuja, Rainer Adelung, Anthony P. F. Turner, Ashutosh Tiwari (Manuscript)

Contribution: Designed and performed all experimental work except theoretical calculations and material synthesis. Wrote whole paper and contributed to the final editing of the manuscript.

\section{Paper IV}

\section{Switchable Bioelectronics}

Onur Parlak, Anthony P. F. Turner

Biosensors and Bioelectronics, 2015 (In press) doi:10.1016/j.bios.2015.06.023

Contribution: Wrote large part of the first draft and contributed to the final editing of the manuscript. 


\section{Paper V}

On/Off-Switchable Zipper-Like Bioelectronics on a Graphene Interface

Onur Parlak, Anthony P. F. Turner, Ashutosh Tiwari

Advanced Materials, 2014, 26, 482

Contribution: Designed whole work and performed all experimental work. Wrote whole paper and contributed to the final editing of the manuscript.

\section{Paper VI}

pH-induced on/off-switchable Graphene Bioelectronics

Onur Parlak, Anthony P. F. Turner, Ashutosh Tiwari

Journal Materials Chemistry B (Accepted)

Contribution: Designed whole work and performed all experimental work. Wrote whole paper and contributed to the final editing of the manuscript.

\section{Paper VII}

\section{Light-triggered On/off-switchable Graphene-based Bioelectronics}

Onur Parlak, Selim Beyazit, Mohammed J. Jafari, Bernadette Tse Sum Bui, Karsten Haupt, Ashutosh Tiwari, Anthony P. F Turner

(Submitted)

Contribution: Designed whole work and performed all experimental work together with second author. Wrote whole paper and contributed to the final editing of the manuscript.

\section{Paper VIII}

\section{Programmable Bioelectronics in a Stimuli-encoded 3D Graphene}

Onur Parlak, Selim Beyazit, Bernadette Tse Sum Bui, Karsten Haupt, Anthony P. F Turner, Ashutosh Tiwari

\section{(Submitted)}

Contribution: Designed whole work and performed all experimental work. Wrote whole paper and contributed to the final editing of the manuscript. 


\section{PAPERS NOT INCLUDED}

\section{Papers}

Self-reporting Micellar Polymer Nanostructures for Optical Urea Biosensing

Suresh K. Shukla, Onur Parlak, Sudeesh Shukla, Sachin Mishra, Anthony P. F. Turner, Ashutosh Tiwari

Industrial \& Engineering Chemistry Research, 2014, 20, 8509

Anamalous Transmittance of Polystyrene/ceria Nanocomposites at High Particle Loadings

Onur Parlak, Mustafa M. Demir

Journal Materials Chemistry C, 2013, 1, 290

Sorption of Uranyl Ions by Amidoximated Polyacrylonitrile Fibers under Continous Flow

Nesrin Horzum, Talal Shahwan, Onur Parlak, Mustafa M. Demir

Chemical Engineering Journal, 2013, 213, 41

Book review

Advanced Synthetic Materials in Detection Science, (Edited by S. Reddy)

Biosensors and Bioelectronics, 2015, 65, 41 


\section{CONFERENCES}

\section{Keynote Talk}

Stimuli-encoded Bioelectronic Sytems

Onur Parlak, Ashutosh Tiwari, Anthony P. F. Turner

Advanced Materials World Congress, 23-26 August 2015, Stockholm, Sweden

\section{Invited Talks}

Switchable Bioelectronics

Onur Parlak, Ashutosh Tiwari, Anthony P. F. Turner

SPIE, Biosensing and Nanomedicine VIII, 9-13 August 2015, San Diego, California, United States

Switchable Bioelectronics on a Graphene Interface

Onur Parlak, Ashutosh Tiwari, Anthony P. F. Turner

World Congress on Biosensors, 27-30 May 2014, Melbourne, Australia

\section{Oral Presentations}

Stimuli-responsive Interfaces for Bioelectronics

Onur Parlak, Ashutosh Tiwari, Anthony P. F. Turner

$2^{\text {nd }}$ International Biosensor Congress, 10-12 June 2015, Izmir, Turkey

Stimuli-encoded Switchable Modulation of Bioelectrocatalytic Graphene

Onur Parlak, Ashutosh Tiwari, Anthony P. F. Turner European Cooperation Science and Technology (COST) Bio-inspired Material Conference, 20-21 October, 2014, Bilkent University, Ankara, Turkey

On/Off Switchable Bioelectronics on a Graphene Interface

Onur Parlak, Ashutosh Tiwari, Anthony P. F. Turner

Advanced Materials World Congress, 16-19 September 2013, Izmir, Turkey 


\section{Poster Presentations}

Switchable Graphene Interface for Biosensing and Energy Applications

Onur Parlak, Ashutosh Tiwari, Anthony P. F. Turner

ICREA-Workshop on Graphene Biosensors, 25-26 May 2015, Barcelona, Spain

Structuring of $\mathrm{Au}$ Nanoparticle Array on Two-dimensional $\mathrm{WS}_{2}$ Interface for Electrochemical Biosensing

Onur Parlak, Prethi Seshadri, Ashutosh Tiwari, Anthony P. F. Turner

World Congress on Biosensors, 27-30 May 2014, Melbourne, Australia

Towards a Cholesterol Biosensor Based on Gold Nanoparticle-functionalised Graphene

Onur Parlak, Ashutosh Tiwari, Anthony P. F. Turner

Label-free Technologies: Advances and Applications, 1-3 November 2012, Amsterdam, Netherlands 


\section{ACKNOWLEDGEMENTS}

First of all, I would like to say that this thesis would not be possible without the help, support and contributions from the people around me. Particularly, I would like to express my gratitude to;

Anthony P. F. Turner, my supervisor for giving me the opportunity to pursue my $\mathrm{PhD}$, and so much freedom to evolve as a scientist in your group.

Ashutosh Tiwari, my co-supervisor, for your help and advice.

Special thanks to Stefan Klinström, director of Forum Scientium, for great support and encouragement at the critical points in the early stage of my $\mathrm{PhD}$.

Anette Andersson, for taking care of all my administrative problems with neverending patience.

All the present and former members of the Biosensors and Bioelectronics group for friendship, discussions and of course fikas! Especially, I would like to thank Valerio Beni and Mikhail Vagin for giving me valuable research tips about electrochemistry and being patient with all of my questions. Also, I would like to thank Martin Mak, Lokman Uaun and Edwin Jager for advice, sharing your knowledge and experiences during this time. I would like to thank also my office-mates, Ting-Yang Nilsson and Hirak Patra and some of my former and present lab-mates, Leila Kashefi, Anıl İncel, Mike Zhybak, Erdoğan Özgür Mohsen Golabi and Nisar UI Khaliq and many others that I did not mention here for creating nice atmosphere both in the office and laboratory.

Thanks to all our collaborators around the world, especially Selim Beyazt, Karsten Haupt, and Yogendra K. Mishra for sharing your experience and valuable materials with us!

I would like to thank two very important people in my career, first my primary school teacher Jale Lop and my supervisor during master study, Mustafa M. Demir who initiated and helped to develop my passion about science. 
My family, but especially latest members my nephews Mira and Doruk for making my vacations full of amusement.

Finally and most importantly :) my wife, Alina Sekretaryova, thank you for your support, encouragement, patience and unwavering love and wonderful life in last three years. Definitely, I could not succeed with any of this without you, Thanks for being near to me! 


\section{ABBREVIATIONS}

DNA Deoxyribonucleic acid

DLVO Derjaguin, Landau, Vervey, Overbeek

EDL Electrical Double Layer

IP Isoelectronic Point

SAM Self-assembled Monolayer

MO Molecular Orbital

NP Nanoparticle

AFM Atomic Force Microscopy

TEM Transmission Electron Microscopy

XRD X-ray Diffraction

SEM Scanning Electron Microscopy

FAD Flavin Adenine Dinucleotide

PQQ Pyrroloquinoline Quinone

GDH Glucose Dehydrogenase

QD Quantum Dots

AChE Acetylcholine

CNT Carbon Nanotube

HOPG Highly Ordered Pyrolytic Graphite

DOS Density of State

TMD Transition Metal Dichalcogenide

ATRP Atom Transfer Radical Polymerisation

ROMP Ring-opening Metathesis Polymerisation

RAFT Reversible Addition Fragmentation Chain Transfer

NMRP Nitroxide-mediated Radical Polymerisation

SPMA Spiropyran Methacrylate

MC Merocyanine

ECM Extra Cellular Matrix

MRI Magnetic Resonance Imagining

PPy Polypyrrole

PAA Polyacrylamide

PNIPAAM Poly- $N$-isopropyl acrylamide 


\section{TABLE OF CONTENTS}

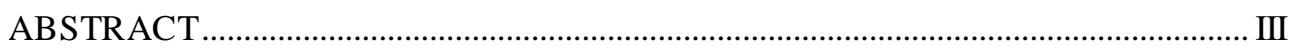

POPULÄRVETENSKAPLIG SAMMANFATTNINGS ............................................... V

LIST OF PUBLICATIONS INCLUDED IN THIS THESIS ............................................VII

LIST OF PUBLICATIONS NOT INCLUDED IN THIS THESIS ..................................IX

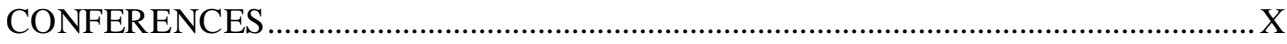

ACKNOWLEDGEMENTS .............................................................................. XIII

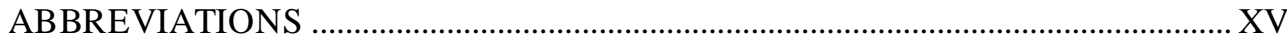

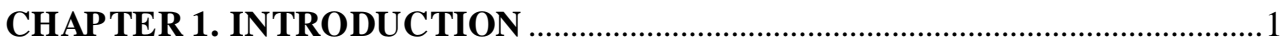

1.1. Nanomaterials and Bioelectronics....................................................................

1.2. Aim and Outline of the Thesis ...........................................................................2

CHAP TER 2. NANOBIOELECTRONICS ………………………………….........

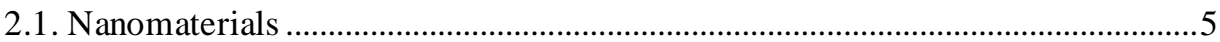

2.2. Physicochemical Interactions at Nano-interfaces .................................................

2.2.1. Colloidal Forces........................................................................................

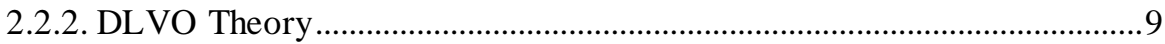

2.2.3. Surface Charge ............................................................................................11

2.3. Interfacing Nanomaterials for Bioelectronics.....................................................17

2.3.1. Conjugated Polymers ..................................................................................18

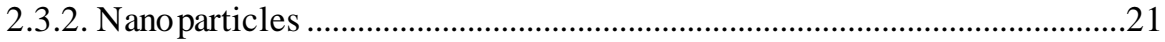

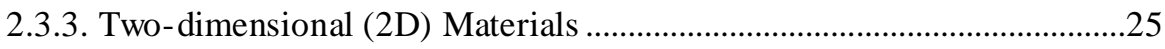

CHAP TER 3. SWITCHABLE B IOELECTRONICS...............................................

3.1. Switchable Bio-interfaces .................................................................................3

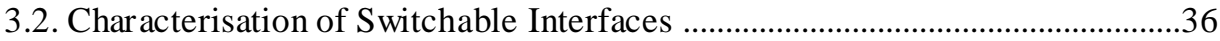

3.3. Physically-stimulated Systems .........................................................................

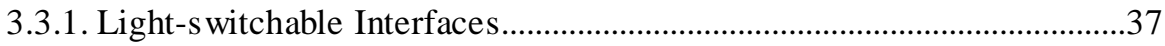

3.3.2. Temperature-s witchable Interfaces ...........................................................38

3.3.3. Electrically-switchable Interfaces ..............................................................39 
3.3.4. Magneto-switchable Interfaces..................................................................40

3.4. Chemically-stimulated Systems .....................................................................

3.5. Programmable Bioelectronics...........................................................................

3.5.1. Enzyme-based Systems for Biocomputing .................................................42

3.5.2. Programmable Enzyme-based Biocatal ytic Systems..................................43

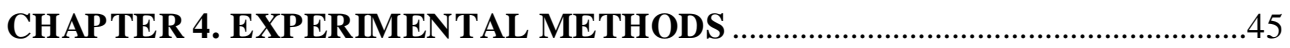

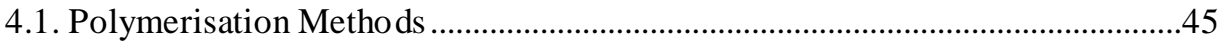

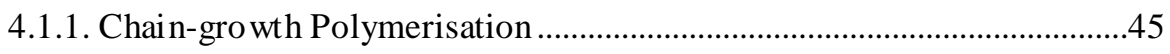

4.1.2. Step-gro wth Polymerisation..........................................................................47

4.2. Enzyme Immobilisation Methods .........................................................................49

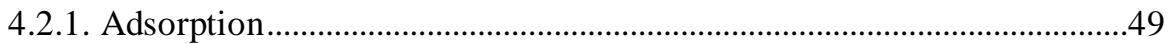

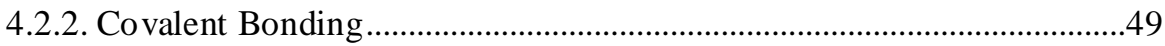

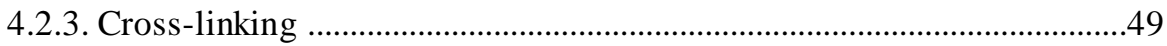

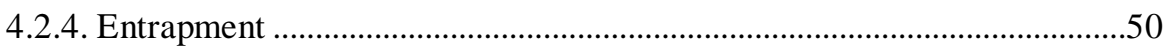

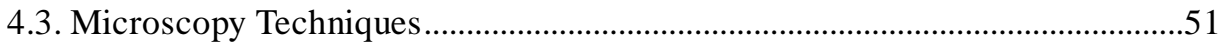

4.3.1. Transmission Electron Microscopy (TEM) …………………………........51

4.3.2. Scanning Electron Microscopy (SEM) ......................................................51

4.4. Electrochemical Methods ……………………………………………………....52

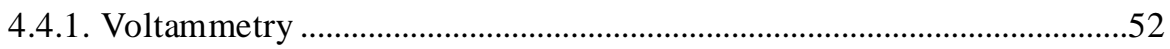

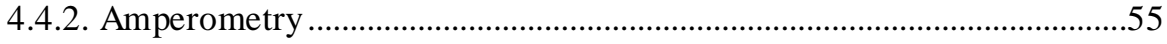

4.4.3. Impe dance Spectroscopy ........................................................................56

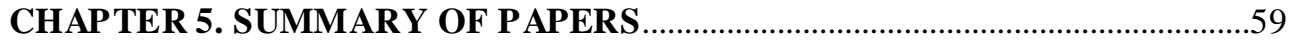

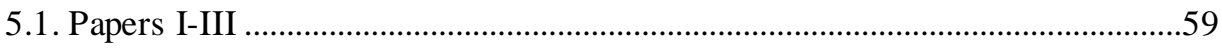

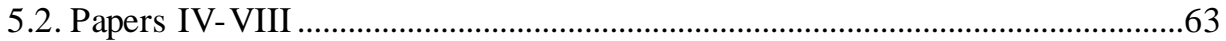

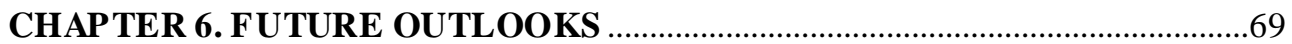

REFERENCES ……………………………………………………………….....

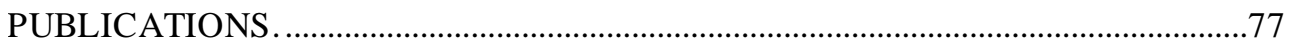




\section{INTRODUCTION}

\subsection{Nanomaterials and Bioelectronics}

Bioelectronics is a rapidly progressing interdisciplinary research field comprising the mutual integration of the living and artificial worlds, where biological and electronic systems are merged together to respond to long-standing needs and fundamental questions about living systems. ${ }^{1,2}$ In parallel with technological achievement in the area of electronics, the area offers significant biochemical and biotechnological progress in the design of novel biomaterials by providing unique ways to synthesise and/or engineer new biomolecules. These novel materials provide wide range of possibilities for their integration with electronic elements. ${ }^{3}$

Many important advances have been achieved in the field of bioelectronics in last century. ${ }^{4}$ It has been proved that electronics can be integrated with biotechnology. ${ }^{5}$ To date several types of bio devices have been developed and made significant contributions for the realisation of this vision. However, there are still many technical challenges existing in these devices at both the lab-scale and for commercial applications. ${ }^{6}$ Researchers have continuously tried to overcome technical challenges to create more precise platforms for both fundamental and applied studies.

One of the crucial components of this vision is the absence of functional interfaces to overcome existing problems in attempts to open up new aspects and to bring practical solutions to bioelectronics. ${ }^{7}$ Progress in materials science and specifically, nanomaterial-based technology, adds new dimensions to the area of bioelectronics. Different classes of materials ranging from metal to the carbonbased systems with different nano-features, provide bio-nanointerfaces with potentially novel electronic properties. The dimensions of various nanomaterials are comparable to those of biomolecules such as enzymes, antigens/antibodies or deoxyribonucleic acid (DNA). Not surprisingly, the conjugation of biomolecules with metal or carbo-based nanostructures often yields hybrid systems with new electronic and optoelectronic properties. Indeed, tremendous progress has accomplished in the realisation of biomolecule-nanomaterial hybrid systems for various bioelectronic applications. The electrical contacting of redox enzymes with electrodes by using metallic nanoparticles, the use of metal nanoparticlenucleic acid conjugates for the catalytic deposition of metals and inducing electrical conductivity between electrodes, the electrochemical analysis of metal ions 
originating from the chemical dissolution of metallic or semiconductor nanoparticle labels associated with DNA, and the photoelectrochemical assay of enzyme reactions by means of semiconductor nanoparticles, represent a few examples that highlight the potential of biomolecule-nanoparticle hybrid systems in bioelectronic design. ${ }^{4}$ Moreover, recently developed carbon-based nanomaterials are also highly desirable for application in bioelectronics research, due to their bio-

compatible and flexible nature. ${ }^{8}$ Applications include wearable electronics (e.g. sensors and actuators) and more recently implantable electronics. Today, for instance, it is almost impossible to imagine flexible electrode materials without carbon-based interfaces (e.g. conductive polymer or graphene) ${ }^{9-12}$ The integration of many different nanomaterials with biomolecules has enormous potential to yield new functional systems that may help to miniaturise biosensors, mechanical devices and electronic circuitry. As a result, it is reasonable to believe that these materials will play ever more important roles in our daily life, from safety, disease diagnostics, and even life sustaining technologies.

The concept of interfacing nanomaterials with bioelectronics is very promising and even though the field does not have a long track record, I believe that there is huge potential to bring "successful generations" of new devices into the world for the future of bioelectronics.

\subsection{Aim and Outline of the Thesis}

The first condition for the successful interfacing between biological and electronic systems is to find the right interface. ${ }^{1,7}$ The interface should be first a "good host" for the biomolecules. For example, if the biomolecule is a protein, the activity should remain as close to the native activity as possible, or if the biomolecule is DNA, the structure and composition should remain more or less same after each interaction. ${ }^{13}$ A second priority, is that the interface should not interfere with the signal moving between biomolecules and the electronic components. For these reasons, the investigation of the interface between nanostructured materials and biomolecules, in order to understand the basics of the dynamic physical and chemical interactions, and kinetic and thermodynamic exchanges, is crucial. In order to yield successful bioelectronic devices, whether at the lab-scale or commercial, we must understand the dynamic forces and molecular components that shape these interactions. ${ }^{14}$ Even though it is not easy to describe all physical and chemical interactions with high certainty, we need to give at least conceptual remarks to guide this investigation.

The second part of this thesis (Chapter 2) is devoted to the exploration of the basics of nanobioelectronics, and more specifically to the understanding of interfacial physicochemical properties at the nano-bio interface. Following on from 
that, many types of nanomaterials are investigated for bioelectronic applications. Special focus is given to recently emerging two-dimensional (2D) materials at the end of this chapter. Here, we not only review the overall progress made to date in using such interfaces and focus on the latest efforts, but also try to understand the basic principles of interactions at nanobiointerfaces, particularly at nanostructured electrode-enzyme interfaces from an electrocatalytic perspective to generate device platforms that integrate bio-interfaces with electronics. In Chapter 2, the main results are presented in three different papers. In papers I, II, and III, different types of interface elements; graphene, $\mathrm{WS}_{2}$ and aerographite were evaluated, respectively. The effect of different surface modifications and morphologies were investigated using different types of materials on the basis of enzyme-based electrochemical sensing of biological analytes.

In the third part of this thesis (Chapter 3), we first focused on the recently emerging field of switchable interfaces for bioelectronic applications, specifically enzyme-based electrochemical bio-catalysis. There has been growing interest in switchable bio-catalysis in response to real-life chemical and physical stimuli as a new platform to understand control and regulation mechanisms underlying physiological processes. Our understanding of natural biochemical interactions and electron transfer phenomena can be furthered by mimicking biochemical reactions and controlling the environment and operations in these models by using external physical stimuli. Modelling of physical interactions of biomolecules in a confined volume has, for example, had significant impact on efficient biocatalysis and functional control by external stimuli physical and chemical stimuli using light, temperature or $\mathrm{pH}$. Bio-molecular interactions involving noncovalent bonding, intermolecular forces and van-der Waals interactions play an important role in bio-catalysis. Hence, the control and regulation of these interactions dominate their function. The systems covered in this chapter are categorised mainly into two groups stimulated by either external physical or chemical stimuli. The former stimuli include light, temperature, magnetic and electrical fields, and the latter involve addition of some specific chemicals, enzymes, change in $\mathrm{pH}$ or ionic strength of the reaction medium. At the end of this chapter, we explore the possibility of constructing of programmable bioelectronics using stimuli-responsive materials to segregate biochemical reactions in a confined space and modulate their function externally. In Chapter 3, the main results and opinions are presented in a review article (paper IV) and 4 different papers (papers $\mathrm{V}$, VI, VII, and VIII).

Paper IV is a review article in which we seek to piece together early breakthroughs and key developments, and highlight and discuss the future of switchable bioelectronics by focusing on bioelectrochemical processes based on mimicking and controlling biological environments with external stimuli. 
In paper V, VI, and VII, we report the fabrication of temperature, light and $\mathrm{pH}$ switchable bio-interfaces using graphene-stimuli-responsive polymer hybrids to control and regulate enzyme-based biomolecular reactions, respectively. Using electrochemical measurements, we demonstrated in each study that interfacial bioelectrochemical properties can be tuned with a modest change in the surroundings of the biomolecules. This responds to a major challenge in nanoscale materials research by regulating the behaviour of switchable bio-interfaces.

In paper VIII, we presented proof-of-principle platforms that lay the foundations for programmable bioelectronic interfaces using two different stimuli on one electrode. Similarly to paper V and paper VI, we designed two different electrodes materials with the same component. These two different electrode designs allow us to programme enzymatic reactions using external physical stimuli. AND and OR gates were realised and characterised. These critical features of an externally controlled Biocomputing system are a requirement for future construction of more complex bio-molecular systems to segregate reaction conditions at a molecular level. It is worth noting that stimuli-encoded material as a trimming element could provide a way to regulate from the 'on-state' to an 'off-state' and/or to programme the rate of biological reactions via slow-to-medium-to-high and vice-versa. 


\section{NANOBIOELECTRONICS}

\subsection{Nanomaterials}

Modern-day life is indispensably dependent on an infinite number of materials. ${ }^{15}$ We have been completely surrounded by technology and its associated materials of macro-, micro- nano- size with various beneficial properties and they have enriched our lives (Figure 2.1). ${ }^{16}$ It is not only in modern days that some of these materials have been around us, and some having been shaping our lives for centuries or even for millennia. Humankind has been continuously interacting with some of them from its entire existence. Form the prehistoric time to the present, people have investigated novel materials and sought to advance them for better performance (Figure 2.2). ${ }^{17}$

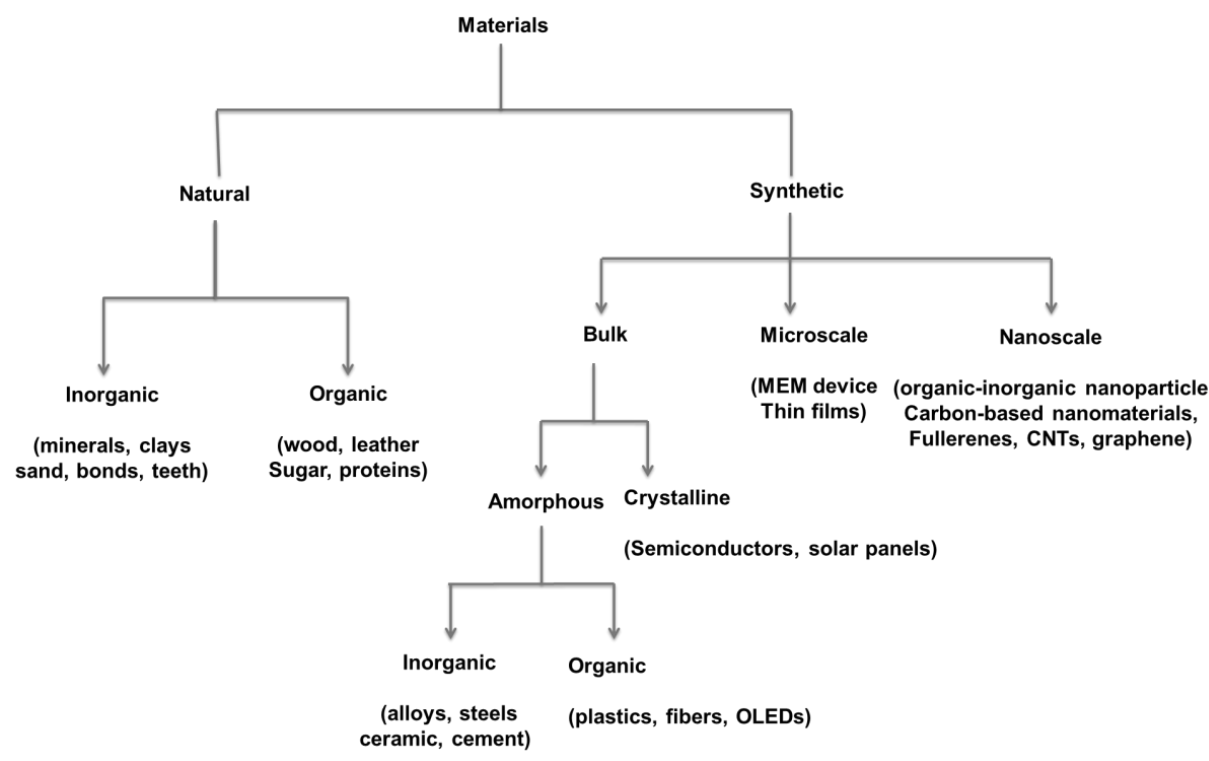

Figure 2.1 Classification of materials. Adapted from Reference ${ }^{17}$

However, among these technologies and developments, nanomaterials have a special place for us and researchers around the world have been paying more and more attention to them. The "nano-", which has etymologically come from the Greek word meaning "dwarf," has been applied to systems whose functions and properties are determined by their size. ${ }^{18}$ The term "nanotechnology" derived 
from technology, encompasses the understanding and engineering of the fundamental physics, chemistry and biology of nanometer scale objects and their functions. In nanotechnology, structures are defined nano as long as any dimension of an object is less than 100 nanometers. ${ }^{19}$

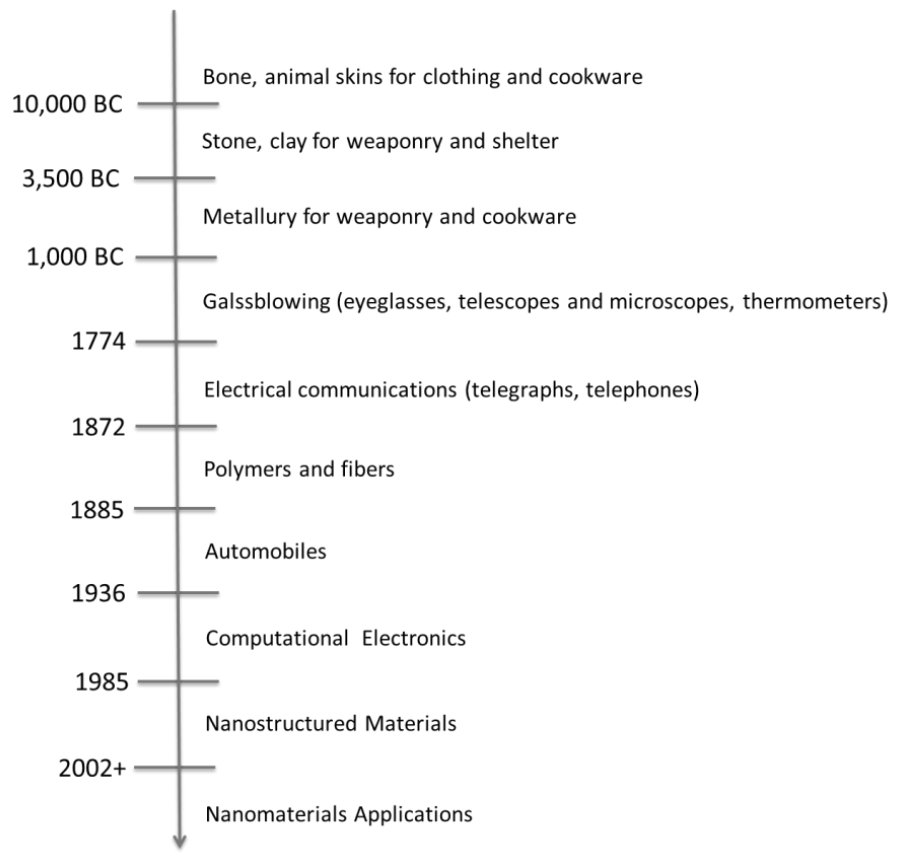

Figure 2.2 Timeline of major developments related to materials science. Adapted from Reference ${ }^{17}$

There are two mainstream synthetic approaches available in nanotechnology. These approaches are categorised as "bottom-up" which emphasises the method of building up from molecules and nanostructures, and "top-down," which simply refers to miniaturisation of bulk materials or structures. ${ }^{20}$ However, Nano- is not usually used to refer to objects simply at the molecular scale. At the nanoscale, material properties are dominated by surface energy and the surface properties, which are different from those of bulk counterparts. ${ }^{21}$ The hierarchy of scales, both spatial and temporal, is represented in the Table 2.1.

The field of nanotechnology is not only growing in terms of fabrication and miniaturisation of materials, but is also toadied by the development of related technologies, such electron microscopy, to observe nanoscale events and individual atoms or molecules and to manipulate them with high spatial precision, as an alternative to the classical surface and colloidal chemistry approaches. ${ }^{22}$ All these 
fabrication techniques together with their related characterisation methods, help us to understand and define surfaces and interfaces at nanoscales and to understand the evolution and dynamics of these structures at different levels. Another promising area is developing in parallel with biotechnology. In nature, all biological assemblies and processes almost invariably take place at the nanoscale, whether across membranes or at interfaces. This inspiration from nature, in future may bring novel bio-molecular materials with unique physical and chemical properties. There is still much to discover about improving periodic arrays of biomolecules and biological templates, and how to exploit the differences between biological and non-biological self-assembly. However, we are brought closer to understanding the bio-molecular world by interfacing them with nanomaterials.

Table 2.1 The hierarchy of spatial and temporal scales. ${ }^{18}$

Scale Quantum Atom/nano Mesoscopic Macroscopic

\begin{tabular}{lllll}
\hline Length (meters) & $10^{-11}-10^{-8}$ & $10^{-9}-10^{-6}$ & $10^{-6}-10^{-3}$ & $>10^{-3}$
\end{tabular}

Time (seconds) $\quad 10^{-16}-10^{-12} \quad 10^{-13}-10^{-10} \quad 10^{-10}-10^{-6} \quad>10^{-6}$

\subsection{Physicochemical Interactions at Nano- interfaces}

The interfacing of nanomaterials with biomolecules such as proteins, DNA, cells and membranes, brings a wide range of interactions at the interface, that depend mainly on colloidal forces and dynamic physical and chemical interactions, into focus. ${ }^{14}$ These interactions play an important role in bio-catalytic processes particularly for the sensing of physiological analytes. ${ }^{23}$ The investigation of various nanostructured interfaces allows us to develop a strong relationship between nanomaterials and biomaterials and their structures and activities, which are mainly determined by size, shape and the surface characteristics of the materials. ${ }^{24} \mathrm{We}$ believe that this knowledge is crucial from the perspective of efficient use of nanomaterials for applications in bioelectronics.

\subsubsection{Colloidal Forces}

Matter can exist in three main states: solids, liquids or gases. ${ }^{25}$ However, when one of these states is dispersed in one another, we need to refer to a "colloidal system". ${ }^{26}$ Colloidal systems such as aerosols, emulsions and suspensions have 
special physical properties that have great practical importance for both fundamental and applied studies. ${ }^{27}$

In non-ideal systems, the particles in dispersion may come closer to each other due to high surface energy and can even stick to one another and form aggregates of successively increasing size, which may eventually collapse by gravitational forces. ${ }^{28}$ There are number of medium-stages of non-stable systems from ideal to phase separation (Figure 2.3.). Once the particles start to aggregate, each particle and the process are called as flocculants and flocculation, respectively. The flocculants sometimes form sediment or undergo phase separation. ${ }^{29}$ If these flocculants transform to a much more dense form, the process is called as coagulation, which usually finalises either by sedimentation or creaming, depending on the density of the particles. ${ }^{30}$ The terms flocculation and coagulation have often been used interchangeably. Usually coagulation is irreversible whereas flocculation can be reversed by the process of de-flocculation. ${ }^{31}$

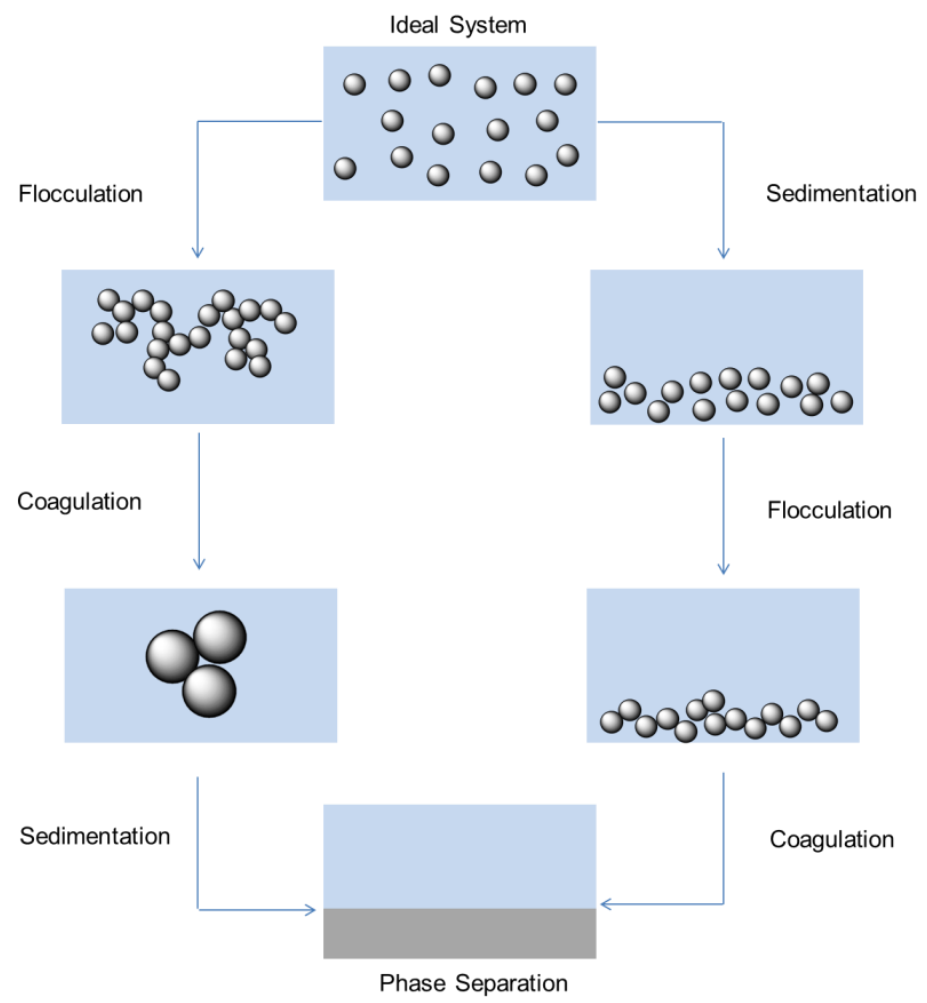

Figure 2.3 Schematic illustrations of various stages of colloidal systems from ideal to phase separation. Adapted from Reference ${ }^{30}$ 


\subsubsection{DLVO Theory}

Derjaguin, Landau, Vervey, and Overbeek (DLVO) introduced a theory which gives a quantitative description of aggregates or so called colloidal stability in aqueous solution and the interactions between charged surfaces and a liquid medium. ${ }^{32}$ The theory is mainly established based on the net interaction between attractive (Van der Waals) and repulsive forces on in colloidal systems. ${ }^{33}$ The theory defines that the stability of a colloidal system depends on van der Waals attractive $\left(\mathrm{V}_{\mathrm{A}}\right)$ and electrical double layer repulsive $\left(\mathrm{V}_{\mathrm{R}}\right)$ forces between particles as they approach each other due to the Brownian motion. Here, the total potential energy function $\left(\mathrm{V}_{\mathrm{T}}\right)$ is described by the sum of attractive $\left(\mathrm{V}_{\mathrm{A}}\right)$ and repulsive $\left(V_{R}\right)$ contributions plus potential energy comes from solvent $\left(V_{S}\right)$ as below: ${ }^{34}$

$$
\mathrm{V}_{\mathrm{T}}=\mathrm{V}_{\mathrm{A}}+\mathrm{V}_{\mathrm{R}}+\mathrm{V}_{\mathrm{S}}
$$

Most important is the relation between $\mathrm{V}_{\mathrm{A}}$ and $\mathrm{V}_{\mathrm{R}}$, which are the attractive and repulsive contributions, respectively. These forces have larger contribution to net energy and are able to operate over a much larger distance. The attractive forces are defined simply by the following equation;

$$
\mathrm{V}_{\mathrm{A}}=-\mathrm{A} /\left(12 \cdot \pi \cdot \mathrm{D}^{2}\right)
$$

where $\mathrm{A}$ is the Hamaker constant and D is the distance between two individual particles. ${ }^{35}$ The repulsive potential however, is a far more complex function as below;

$$
\mathrm{V}_{\mathrm{R}}=2 \cdot \pi \cdot \varepsilon \cdot \mathrm{a} \cdot \zeta^{2} \cdot \exp (-\kappa \mathrm{D})
$$

where $\mathrm{a}$ is the particle radius, $\pi$ is the solvent permeability, $\kappa$ is a function of the ionic composition and $\zeta$ is the zeta potential. ${ }^{36}$ 


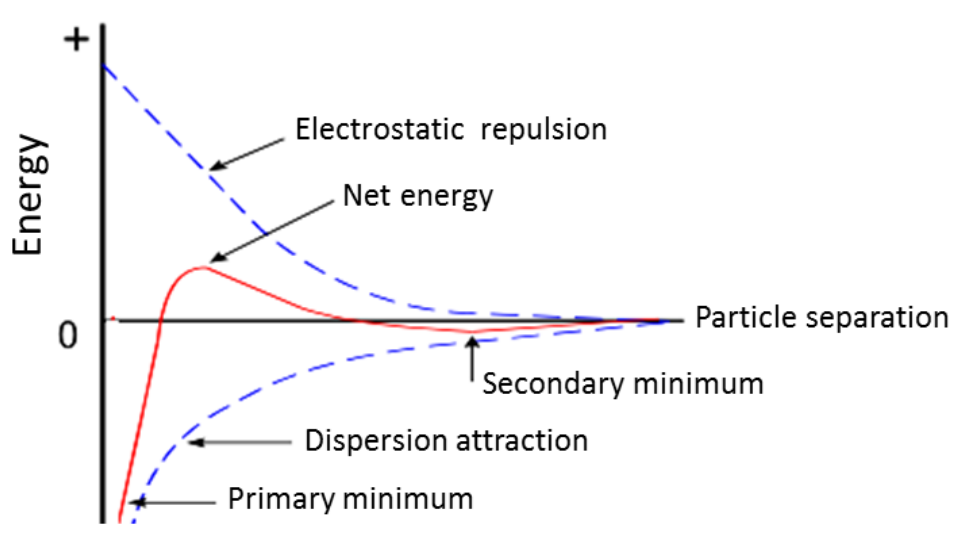

Figure 2.4 Schematic of free energy and particle separation relation according to DVLO theory. Adapted from Reference ${ }^{30}$

As can be seen in Figure 2.4, the energy barrier is the reason the repulsive force does not allow two particles to approach and adhere to one another in solution. However, once the particles collide with each other with high energy, which is enough to overcome that barrier, the attractive forces pull them into contact where they adhere strongly to one another. In this way, if the particles have a sufficiently high repulsion, the dispersion can resist flocculation and the colloidal system can be stable. However if a repulsion mechanism does not exist then flocculation or coagulation can easily occur. ${ }^{37}$

In the presence of high salt concentrations (reducing zeta potential), the colloidal system can have the chance of a secondary minimum, where weaker and reversible adhesion between particle occurs. ${ }^{38}$ These weak particle combinations (flocculants) are sufficiently stable and not to be broken easily by Brownian motion. However, they may only disperse under applied external forces such as strong mixing. In order to maintain the stability of colloidal systems, the contributions from repulsive forces should be dominant. There are two fundamental mechanisms that determine stability of dispersion stability. ${ }^{39}$ 


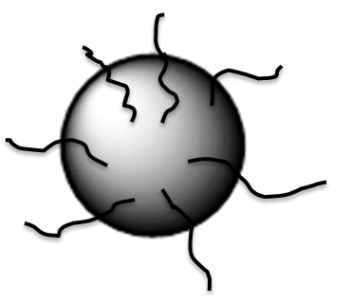

Steric stabilization

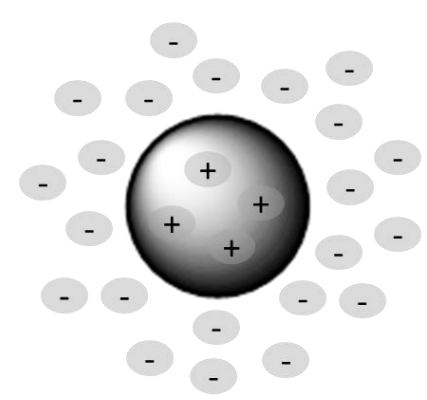

Electrostatic stabilization

Figure 2.5 The steric and electrostatic stabilisation of colloidal systems. Adapted from Reference ${ }^{30}$

One of these approaches is called steric stabilisation (Figure 2.5). In this case, relatively large molecules such as polymer chains are adsorbed on the particle surface and suppress the aggregation by reducing the surface energy of each individual particle. Here, the particles are separated from each other by steric repulsion, which hinders the effect of the weak van de Waals forces. Another system is called electrostatic or charge stabilisation. ${ }^{40}$ In this technique, the particle interaction is suppressed by the distribution of charged species in the colloidal medium. Both mechanisms have advantages over their counterparts. For example, steric stabilisation is relatively easy to achieve and only requires the onestep addition of a suitable polymer, but it can be difficult to subsequently flocculate the system when required. The polymer approach can be expensive and in some cases the polymer is undesirable, may shrink depending on environmental conditions and can lead to deformation. Electrostatic or charge stabilisation, on the other hand, has several benefits such as reversibly stabilising or flocculating the system by simply adjusting the concentration of ions in the medium and is, in addition, a relatively inexpensive process. ${ }^{41}$

\subsubsection{Surface Charge}

Most colloidal dispersions bear an electrical charge in aqueous solutions. The origin of this surface charge is mainly based on the particle and the medium surrounding the particles.

\section{Ionisation of Surface Groups}

One reason for the negative charge on the particle surface is the dissociation of acidic groups. However when the surface is basic, the surface evolves to a positively charge. ${ }^{42}$ In both cases, the magnitude of the surface charge is directly re- 
lated to the strengths of the surface groups and the $\mathrm{pH}$ of the medium. As illustarted in Figure 2.6a-b, the surface charge can be reduced by supressing the surface ionisation by adjusting the $\mathrm{pH}$ of the medium. The reducing $\mathrm{pH}$ yields negatively charged surface, or positively charged surface by increasing the $\mathrm{pH}{ }^{43}$

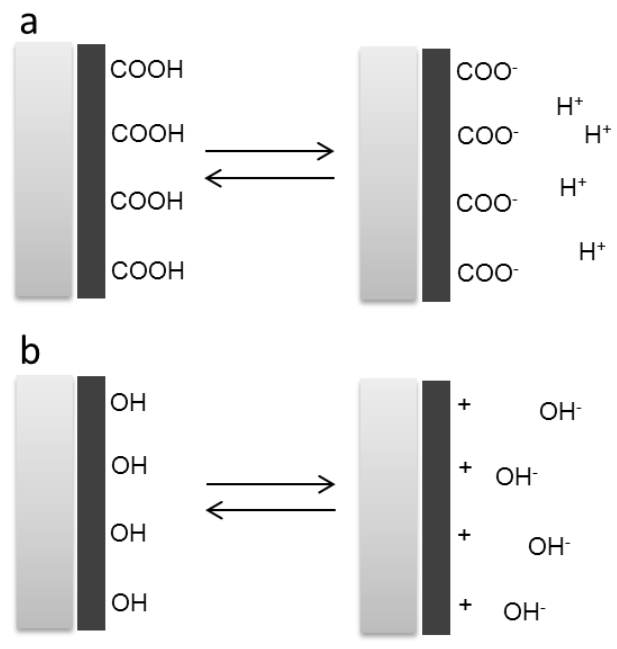

Figure 2.6 A schematic illustration of the origin of surface charge by ionisation of acidic (a) and basic groups (b). Adapted from Reference ${ }^{30}$

\section{Adsorption of charged species}

Charged species such as surfactant ions, may adsorb on the surface of a particle, which gives a positively charged character to the surface in the case of a cationic surfactant, and a negatively charged surface in the case of an anionic surfactant. ${ }^{44}$

\section{Electrical Double-layer}

The electrical double layer (EDL) is a net charge formed around each surface, which affects the distribution of ions in the interfacial medium. The forming of EDL results increased a concentration of opposite charge ions around the surface. $^{45}$

\section{Zeta Potential}

The liquid layer around each surface hypothetically contains two parts (Figure 2.7). The inner region closer to the surface is called the Stern layer. ${ }^{46}$ In this layer, the ions are strongly bound to the surface. However, in the diffuse layer, ions are less firmly associated with the surface. In the diffuse layer, the ions and particles stay in a stable form where the particles have random motions due to gravita- 
tional or any other external forces. ${ }^{47}$ Those ions beyond the boundary stay with the bulk dispersant. The potential at this boundary or surface of hydrodynamic shear is named the zeta potential. The zeta potential is a common indication to understand the stability of the colloidal system. There are some quantitative values that give a direct answer the suspension characteristics. For example, if suspension has a very large negative $(<-30 \mathrm{mV})$ and $(>+30 \mathrm{mV})$ positive zeta potential, the particles in suspension tend to repel each other and this reduces possibility of aggregation or flocculation. However, when the system has low zeta potential, there will be no forces to stabilise the particles and eventually the tendency to flocculation will increase. ${ }^{48}$

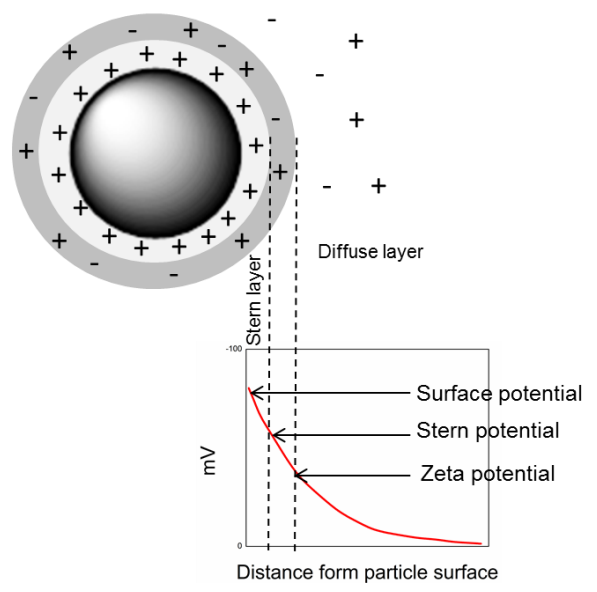

Figure 2.7 Schematic representation of zeta potential. Adapted from Reference ${ }^{30}$

\section{Factors Affecting Zeta Potential}

The $\mathbf{p H}$ of the medium is one of the most important factors that affects the zeta potential of colloidal systems in aqueous solutions. For example, when more alkali is added to the suspension with a negative zeta potential, the particles tend to acquire more negative charge. ${ }^{49}$ The turning of medium to acidic conditions results in build-up of positive charge on the same surface. Eventually, the zeta potential versus $\mathrm{pH}$ curve intercept will be positive at low $\mathrm{pH}$ and lower or negative at high $\mathrm{pH}^{50}$ During this transition, the zeta potential will be " 0 " at a certain $\mathrm{pH}$. This point is called the isoelectric point (IP) where the colloidal system is least stable and is very important from a practical consideration of colloidal systems. As can be seen in Figure 2.8, the IP of the sample is at approximately pH 5.5. The plot can be used to predict that the sample should be stable at $\mathrm{pH}$ values less than 4.5 , due to there being sufficient positive charge on the surface, and at greater than $\mathrm{pH} 7.5$, due to the surface bearing sufficient negative charge. The 
unstable state for the dispersion would be expected at $\mathrm{pH}$ values between 4.5 and 7.2 as the zeta potential values are between +30 and $-30 \mathrm{mV}$.

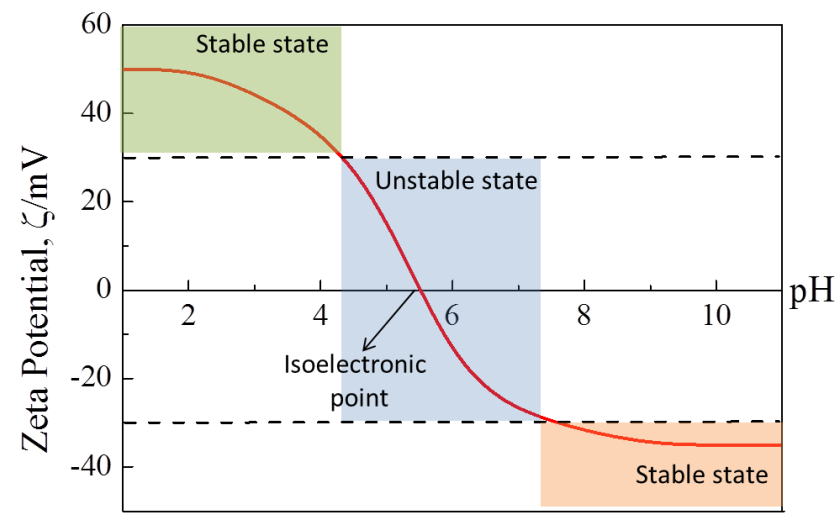

Figure 2.8 The schematic illustration of typical zeta potential versus $\mathrm{pH}$ plot.

Another important parameter affecting the zeta potential is the thickness of the double layer. ${ }^{46}$ The double layer thickness can be calculated from the ionic strength of the medium and depends on the concentration of ions. The high ionic strength and high valence state of the ions makes the double layer more compressed. There are two main mechanisms for interaction between ions and the surface;

I. Non-specific ion adsorption (does not affect IP), which does not have any contribution to the isoelectric point

II. Specific ion adsorption (contributes to the IP).

\section{Electro-kinetic Effects}

The interaction between electrical charges on the particle surface with applied electrical field has an important effect on the electro-kinetics. There are four distinct effects depending on the way in which the motion is induced. ${ }^{51}$

Electrophoresis: Electrophoresis is described as the movement of charged particles relative to the liquid under the influence of an applied electrical field. When an electric field is applied to the system, the charged particles move toward the oppositely charged electrode. When equilibrium is reached between these two opposing forces, the particles move with constant velocity. ${ }^{52}$ The velocity of the particles is dependent on the strength of applied electric field or voltage gradient, the dielectric constant of the medium, the viscosity of the medium and the zeta 
potential. The velocity of a particle is known as its electrophoretic mobility. The relation between zeta potential and electrophoretic mobility is described by the following equation:

$$
\mathrm{U}_{\mathrm{E}}=2 \cdot \varepsilon \cdot \mathrm{z} \cdot \mathrm{f}(\kappa \mathrm{a}) / 3 \eta
$$

where $\mathrm{U}_{\mathrm{E}}=$ electrophoretic mobility, $\mathrm{z}=$ zeta potential, $\varepsilon=$ dielectric constant, $\eta$ $=$ viscosity and $\mathrm{f}(\kappa \mathrm{\kappa})=$ Henry's function. ${ }^{53}$ The units of $\kappa$, termed the Debye length, are reciprocal length and $\kappa^{-1}$ is often taken as a measure of the "thickness" of the electrical double layer. The "a" term describes the radius of the particle and ка measures the ratio of the particle radius to electrical double layer thickness.

The other effects are: electro-osmosis, which is related the movement of a liquid relative to a stationary charged surface under the influence of an electric field; streaming potential, which results from the electric field generation when a liquid is forced to flow past a stationary charged surface; and sedimentation potential, which results from charged particle sedimentation under an applied electric field. $^{54,55}$

\section{Interactions at the Nano-bio Interface}

After summarising the general concepts of colloidal forces, the different aspects of nano-bio interfaces and related interactions will now be categorised. There are three main types of interaction at the nano-bio interface (Figure 2.9):

I. surfaces of nanomaterials which are defined by physicochemical composition;

II. solid-liquid interfaces where nanomaterials meet their surroundings;

III. nanomaterials in contact with the biological substrate.

Physicochemical composition of a nanomaterial's surface: the chemical composition, surface functionalisation, shape, porosity, crystallinity and defects, and wettability of nanomaterials play a key role in determining their interfacial properties. ${ }^{56}$ The other important parameters such as surface charge, degree of stability and solubility, which are determined by the physical and chemical properties of the suspending media, have a strong impact on the interaction of nanomaterials with the surrounding medium and biological elements. These parameters affect mainly: 


\section{Suspending media}

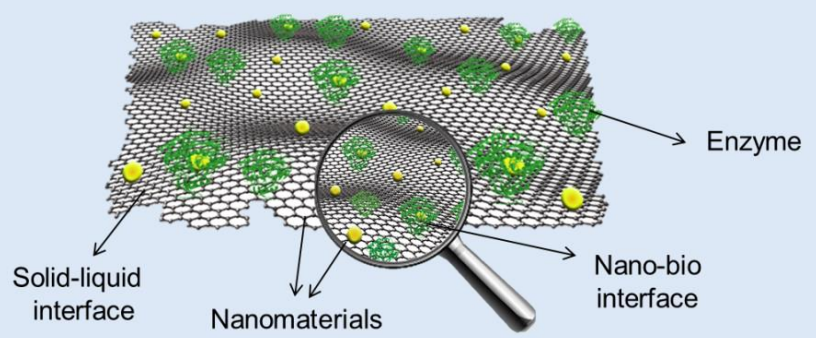

Figure 2.9 Schematic representation of the interface between nanomaterials and biomolecules.

I. adsorption of natural or synthetic organic molecules and biomaterials;

II. formation of the double-layer, which is crucial for electrochemical applications;

III. reduction of the surface energy.

Forces at a solid-liquid interface: In addition to surface composition and properties of nanomaterials, understanding of the interfacial forces at a solid-liquid interface is also crucial. ${ }^{57}$ To determine the bulk properties of suspensions such as net-charge and isoelectronic point, steady-state behaviours are usually considered, even though the interface is not steady-state. But the fact is that nano-bio interfaces continuously change as a result of environmental influences. Even though interactions at the interface involve large numbers of forces, the successful use of nanomaterials to achieve measurable outcomes indicates that it is possible to probe the nano-bio interfaces experimentally.

Forces at the nano-bio interface: The interaction between nanomaterials and biomolecules follows some of the same principles as those between colloidal particles. $^{58}$ The van der Waals, electrostatic, hydrophilic/hydrophobic and many others are still applicable, but they require special attention because the cases occur at the nanoscale. Since nanomaterials possess relatively few atoms, forces related with them are highly dependent on the position of their surface atoms and their standard bulk-permittivity functions. 


\subsection{Interfacing Nanomaterials for Bioelectronics}

The miniaturisation of electronic devices and advances in nanomaterial research and production with the application of functional nanomaterials is at the forefront of scientific and industrial attention. ${ }^{56,59}$ The use of nanomaterials as an interface element on their own or as part of a hybrid structure, allows new properties to be exploited in the area of bioelectronics. The different types of nanomaterials, from metal or semiconductor nanoparticles to the $2 \mathrm{D}$ carbon-based structure, have an important ability to provide suitable platforms for interfacing nanomaterials for bioelectronic applications. ${ }^{60}$ The unique physical and chemical properties of nanostructured materials provide an ideal microenvironment for biomolecule immobilisation while retaining their biological activity, and to facilitate electron transfer between the immobilised biomaterials and electrode surfaces. This has led to intensive use of nanomaterials for the construction of electrochemical biodevices with enhanced analytical performance. Advances in these applications require a fundamental understanding of the complex interactions between nanomaterials and bio-systems. ${ }^{61}$ Using this insight, the tools of chemical synthesis can be used to create nanomaterials that interact efficiently and predictably with biosystems including proteins, nucleic acids, cells and tissues. The chapter 2 aims to discuss the overall progress in building such interfaces at the level of biomolecules and focuses on recent efforts to create device platforms that integrate nanomaterials with bioelectronics, especially from an electrochemical biosensing perspective. Here, we try to highlight the use of synthetic materials to improve electrical interfacing between biological systems and electrodes.

In the area of nanobioelectronics, biological systems have been employed as a biochemical transducer of biochemical signals to electronic information. ${ }^{62}$ There have, of course, been many important challenges for the integration of biological and electronic systems whether at lab-scale or in commercial applications. The barriers to charge transport in biological matrices and electron transfer between redox proteins hinder the construction of efficient interaction between abiotic-biotic interfaces, to name just two. ${ }^{63}$

Most microorganisms are naturally able to affect external electron transfer to and/or from an electrode surface. Similarly to the well-established area of electrochemical biosensors, there are three main electron transfer mechanisms are available between microorganisms and electrode surface. ${ }^{64}$ The electron transfer may occur through either direct contact with an electrode, or transfer through conductive wiring between active side of microorganisms and electrode or mediated transport via redox active shuttles. However, the presence of such microorganisms that are able do extracellular electron transfer, is limited. ${ }^{65}$ 
Therefore, there is a special interest in the area of bioelectronics to find general and easy methods that can facilitate electron transfer at biotic-abiotic interfaces. One way is to interface biomolecules and/or microorganisms with novel nanomaterials to achieve efficient electron and charge transport. There are many different materials including small molecules in various forms, such as selfassembled monolayer (SAM) or supramolecular structures, semiconducting or redox-active polymers, inorganic materials and nanoparticles, and carbon-based materials such as graphite, carbon nanotubes or graphene. In following sections, we focus mainly on nanostructured materials based on nanoparticles and carbon based nanomaterials together with semiconducting polymers.

\subsubsection{Conjugated Polymers}

A polymer is described as the combination of a large number of covalently linked monomer, repetitive sub-units. The chemical and physical properties of polymers depend heavily on the chemical and physical nature of monomer units. Conjugated polymers have alternating double and single bonds in their polymeric backbone structures, which provide electrical conductivity. ${ }^{66}$

Even though conjugated polymers are usually employed as an electrodeposited thin films or directly attached as a macromolecules, rather than nanostructured elements, we included conjugated polymers in this section due to their intensive use in the area of biosensors and bioelectronics. ${ }^{67}$ The use of conducting polymers as an interface element between biology and electronics is an exciting area of research in the field of organic electronics. The growing field of organic bioelectronics research includes coupling of organic electronic devices such as electrodes and transistors with biomolecules and requires extensive effort to interface between biotic-abiotic systems. ${ }^{68}$ There are many important features of conducting polymers that make them an ideal interface element:

I. ability to form interfaces with electrolytes that lack intrinsic oxides and dangling bonds;

II. ability to have mixed conduction of electronic and ionic charge carriers at the same time.

III. possession of soft mechanical properties which bring indispensable physical advantages for implanted devices.

IV. ease of solution processing and device fabrication.

The solution processibility and possible flexibility are of crucial importance for designing inexpensive, flexible and or printable single-use devices. These features provide unique opportunities to the scientist in the area of bioelectronics 
to interface electronics with biology and more importantly, help to solve problems related to diagnosis and treatment of biological disorders.

Conductive polymers such as polyaniline (PANI), polypyrolle (PPy), polythiophenes and many other which are not listed in Figure 2.10 have attracted significant attention as an interface material for mediating interfacial charge transport, due to their efficient electron shuttling ability, tuneable redox potential and ability to serve as a support matrix to host biomolecules such as enzymes and cells on an electrode surface. ${ }^{69}$

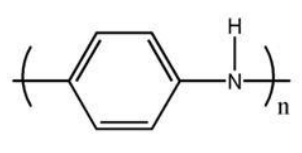

Polyaniline

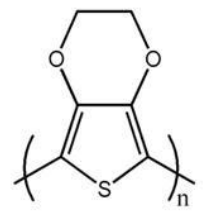

Polyethylenedioxythiophene

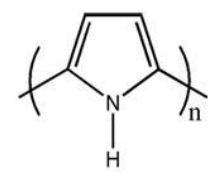

Polypyrrole

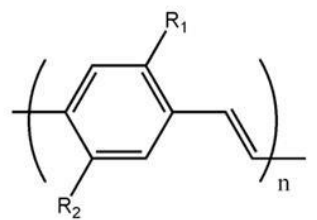

Poly(p-phenylene vinylene)s

Figure 2.10 Illustration of chemical structures of some of conductive polymers.

\section{Molecular and electronic structure in conjugated polymers}

The electrons in an atom are hypothetically fixed within certain electronic orbitals. ${ }^{70}$ Simply, electronic orbitals are described as a region within the atom having highest probability of being found. Atoms bear multiple orbitals. ${ }^{71}$ Each orbital has its own specific energy level and properties, and they are labelled with specific quantum numbers such as $1 \mathrm{~s}, 2 \mathrm{~s}, 2 \mathrm{p}, 3 \mathrm{~s}, 3 \mathrm{p}, 4 \mathrm{~s}, 3 \mathrm{~d}$ etc. The number in these quantum numbers designates principal quantum numbers and the letters represent the orbital angular momentum quantum number (l). Each letter is paired with a specific $l$ value as follow: $\mathrm{s}$ : subshell $=0, \mathrm{p}$ : subshell $=1, \mathrm{~d}$ : subshell $=2$, and $\mathrm{f}$ : subshell $=3$. In addition to these numbers, each orbital is also represented by magnetic quantum number $\left(\mathrm{m}_{\mathrm{l}}\right)$, which can only range from -1 to +1 . This number show how many orbital exist and how many electrons can present in each orbital. $^{72}$

When two atoms come closer to each other, the valence electrons start to interact. The interaction between two valence electrons is basically described by molecular orbitals, which is the linear combination of the atomic orbitals. There are two main types of formation orbital. The bonding molecular orbital is constructive and as a results lower energy is formed. The other is anti-bonding is 
destructive and as a result higher energy is generated (Figure 2.11). When a stable bond between the two atoms is formed, the total energy of the occupied molecular orbitals decreases to a level lower than the energy of the two separate atoms. ${ }^{73}$ In the notations in this Figure, $\sigma$ stands for coaxially symmetric bonds and $\pi$ for non-coaxially symmetric bonds.

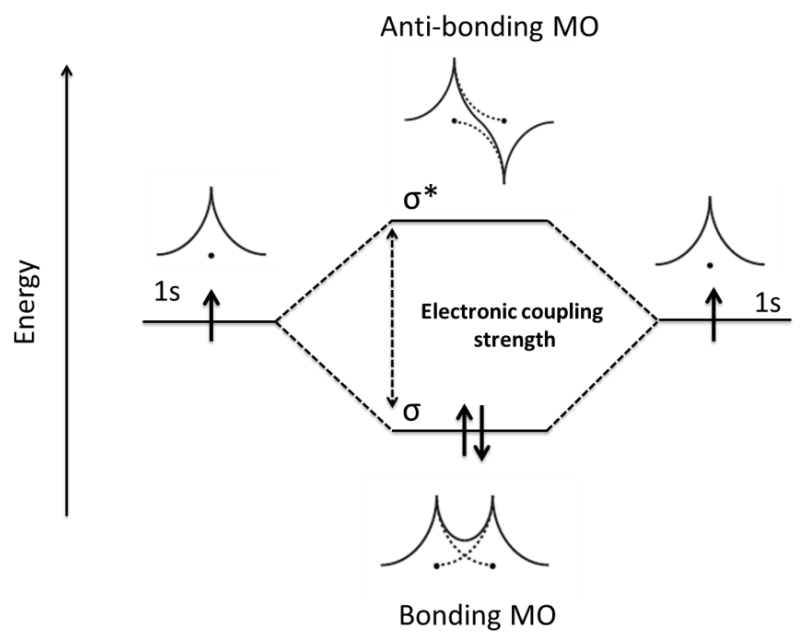

Figure 2.11 The overlapping of two $l s$ atomic orbitals to form two molecular orbitals, one bonding orbital $(\sigma)$ and one anti-bonding orbital $\left(\sigma^{*}\right)$.

The carbon atom is a building block of polymers, has an ability to form a maximum of four single bonds with other atoms. In the ground state, the $1 \mathrm{~s}^{2} 2 \mathrm{~s}^{2} 2 \mathrm{p}^{2}$ orbitals are occupied, however as soon as covalent bonds are formed this configuration changes. When the carbon atom binds to other atoms, the electrons occupy some of the hybrid orbitals. The $s p^{2} p z$ hybridisation, on the other hand, comprises three hybrid orbitals in same plane, which results in the formation of a $\sigma$ bond and one p-orbital is orthogonal to the other plane, which forms a $\pi$-bond. When both types of bond are formed between two atoms, it is referred to as a double bond. In the linear sp hybridisation, two $\sigma$ and two $\pi$ bonds are formed. ${ }^{74}$

The structural characteristic of conjugated polymers is the main reason for their relatively-infinite $\pi$-conjugation system extending over the polymeric backbone. This property gives the polymeric materials directional conductivity along the axis. They usually possess a spatially delocalised, band-like electronic structure. The reason for formation of this band is the splitting of interacting molecular orbitals of the monomer units. The number of molecular orbital is essentially equal to the number of carbon atoms; in this way the energy level is split up 
with increasing chain length. The difference between energy levels decreases as the chain length increases.

The mechanism behind the polymer's conductivity is based on the motion of charged defects in the conjugated backbone. There are two main charge carriers. ${ }^{75}$ The positive carriers are called p-type and negative carriers are called as n-type. They are the products of oxidation and reduction of polymeric backbone, respectively. The polymers form a radical cation upon their oxidation with both spin and charge. These species is defined as a polaron. The cation and radical generates a bound species due to the increasing of the distance in between them and results in the creation of additional higher energy quinoid units. Additionally, the combination of two adjacent polarons forms the lower energy bipolaron, which is more stable than two polarons despite the coulombic repulsion of the two ions. Because of this defect, the boundary between the two moieties can migrate in both directions. Thus there is no significant energy barrier to the process and high carrier mobility leads to the high conductivity of these polymers. ${ }^{75}$

The conductivity of a conducting polymer is directly proportional to the number of charge carriers and their mobility $(\sigma \sim n \mu)$. Due to the large band gap in polymers, $n$ is very small and as a result conjugated polymers are insulators. The polymers can be made conductive by generating a mobile charge via p-type doping (oxidation) or n-type doping (reduction) of the polymeric chains using chemical or electrochemical methods. ${ }^{69}$

\subsubsection{Nanoparticles}

One of the most commonly used interfacing element in the area of bioelectronics is metal and semiconductor-based nanoparticles (NPs) and related nanostructured surfaces. These materials are usually preferred due to their high-surface area, inherently conductive nature and versatile hosting for conjugation with biomolecules compared to bulk materials. They have been employed mainly in catalytic and electro-catalytic applications where they provide a high surface area to mass ratio and promote particular reaction pathways. ${ }^{59}$

A key aspect of the study of interfacing nanoparticles is to prepare and characterise a nanostructured surface of an electrode. There are two main reasons for using support electrodes. Firstly, a support electrode is able to bridge nanoparticles to an external circuit and secondly, an electrode support prevents aggregation of nanoparticles and thus maintains a high surface-to-volume ratio. Various methods are available to incorporate nanoparticles as an interface element between an electrode surface and biomolecules. ${ }^{76}$ The three main approaches available are illustrated in Figure 12: (a) in-situ generation by electrochemical reduction of metal salts ; (b) precise immobilisation of metal salt on the support elec- 
trode and their reduction to metallic form; and (c) chemical attachment of externally synthesised nanoparticles. ${ }^{77}$

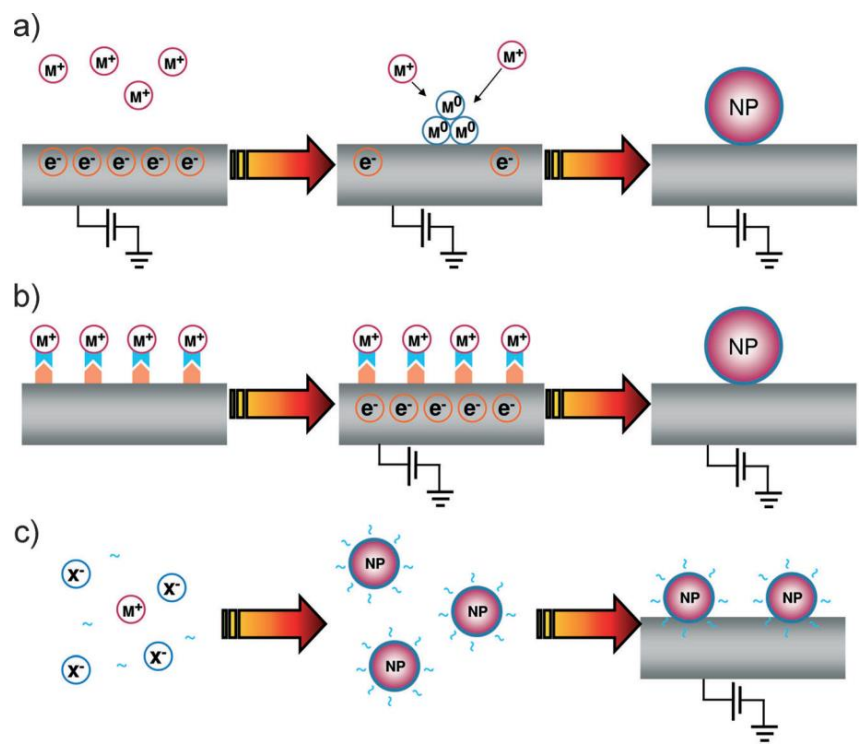

Figure 12 The general formation principals of nanoparticle modified electrodes. Copyright (C) 2014 WILEY-VCH Verlag GmbH \& Co. KGaA, Weinheim. ${ }^{77}$

The first approach includes mainly electrodeposition of nanoparticles from solution onto the bare electrode or onto support electrode modified previously polymeric films (a). Electrodeposition is one of the easiest and practical methods which give the opportunity to tune deposition parameters such as temperature, time, current, potentials and electrolyte composition in order to adjust the size, shape and distribution of nanoparticles on the electrode support. However, this technique is not enough to tune the size of deposited particles and usually yields wide range of size distribution. The second approach includes two successive steps (b). In the first step, metal salts are chemically or electrochemically immobilised on the support electrode. This helps to adjust the spatial distribution of particles and limits the aggregation of individual particles on the electrode surface. In addition, depending on the number of immobilised ions, individual particle size can be easily adjusted. However, the best way of controlling particle size is facilitated by a third method (c). ${ }^{77}$ The colloidal synthesis of nanoparticles allows easy tuning of particle size and shape and also allows the surface of the particle to be modified. The immobilisation of the prepared nanoparticles is also relatively more practical than with other methods. The immobilisation of anchoring groups on the electrode support can facilitate attachment of nanoparticles (with or without modification) with many different methods including click- 
chemistry, thiol chemistry or electrostatic interactions, or the surface having an affinity to the nanoparticles which are to be immobilised.

Following immobilisation or deposition of nanoparticles onto an electrode support, an important step is to characterise the size, shape and distributions of the particles together with the newly generated surface area. Characterisation methods are divided mainly into two-categories: electrochemical and nonelectrochemical. The non-electrochemical methods include scanning electron microscopy (SEM), transmission electron microscopy (TEM), atomic force microscopy (AFM), X-ray diffraction (XRD) and many others. The two types of method are complimentary to each other. For example, although the particle size and shape can be easily revealed by microscopic techniques, it is impossible to obtain information about the electrochemical activity of the surface. In contrast, while electrochemical techniques give information about the active surface area and redox potential of nanoparticles, there is no chance to obtain information about particle size distribution without microscopic techniques. ${ }^{78}$

The deposition or immobilisation of nanoparticles on the electrode support is not enough by itself. There are couple of parameters that should be considered to determine the deposition quality and effects of the nanostructured surface on the interfacial electrochemical properties. The easiest way is to understand the mass transport regime of a nanostructured surface before and after immobilisation/deposition. ${ }^{77}$ A nanostructured surface changes the mass transport regime of amacro-electrode and leads to lower capacitive currents and radial diffusion, compared to planar diffusion at electrode before deposition of nanostructures on the surface. This property allows steady-state currents to be yielded in relatively short periods of time. Before nanostructuring of particles, the overlap between diffusion spheres generates a continuous layer, and the electrode acts as planar electrode (Figure 13a). ${ }^{79}$ However, when nanoparticles are structured on a supporting electrode with relatively good spatial distribution, this generates radial diffusion spheres around each particle (Figure 13b). ${ }^{80}$ Here, the crucial point is to keep the inter-particle distance large enough to keep radial diffusion spheres, otherwise, when the inter-particle distance decreases, each radial diffusion sphere overlaps with others and the surface and diffusion regime becomes more similar to planar behaviour. 

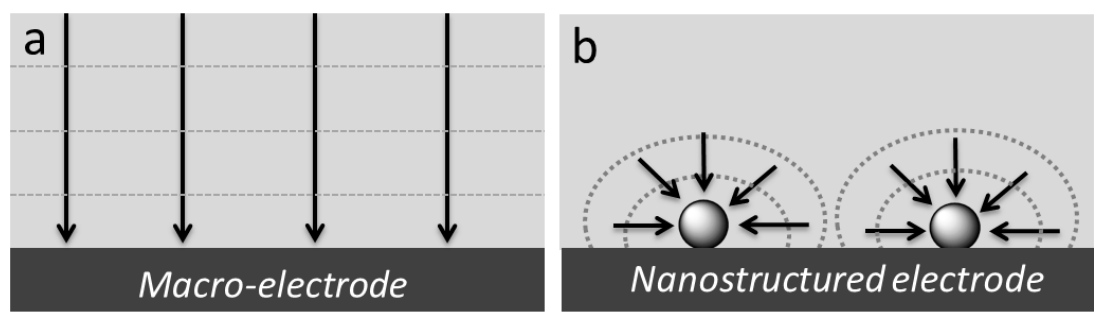

Figure 13 Schematic illustrations of planar (a) and radial (b) diffusion on a macro-electrode. Copyright (C) 2014 WILEY-VCH Verlag GmbH \& Co. KGaA, Weinheim. ${ }^{77}$

Functionalised nanoparticles are commonly preferred since they provide an efficient way to "wire" redox proteins/enzymes for applications in electrochemical biosensors and biofuel cells. In addition, the labelling of oligonucleotides or antigens/antibodies with nanoparticles has been commonly used for electrochemical immunosensors and DNA sensors. Most proteins and redox enzymes lack the ability to communicate directly with the electrode surface. This technical challenge is mainly solved by the tethering of redox-relay groups to the proteins, immobilising of biomolecules on redox-active polymers or using electrontransfer mediators, which all give improved performance. However, some of these proposed methods are not enough to provide efficient electrical contacting of enzymes with the electrode surface due to non-optimal modifications or the lack of appropriate alignment of enzymes in respect of the electrode. One of the possible ways of facilitating of electrical contact between redox-proteins and electrode is to use reconstituted apo-enzyme on relay-cofactor monolayer immobilised electrodes. In this way, researchers have achieved direct electrical communication between the redox centres of the biocatalyst and different electrodes, for example, using reconstituted apo-glucose oxidase on a relay FAD monolayer, and reconstituted apo-glucose dehydrogenase on pyrroloquinoline quinone (PQQ)-modified polyaniline film on the electrode surface. The incorporation of metallic nanoparticles in this process also facilitates controlled electron transfer at the interface. When bioelectrocatalytic electrodes are prepared by coimmobilizing gold nanoparticles and redox enzymes on an electrode surface, efficient electron transfer is not facilitated due to the random and non-optimised positioning of redox proteins. However, when reconstituted apo-flavoenzyme, apo-glucose oxidase, is incorporated together with flavin adenine dinucleotide (FAD) cofactor amino-derivative gold nanoparticles, highly efficient direct contacting of enzyme to the electro surface is achieved. ${ }^{81}$ 
Semiconductor nanoparticles or quantum dots (QDs) have attracted considerable attention due to their tuneable electronic properties as a function of size; the so-called quantum size effect. ${ }^{82}$ Although the synthesis and photo-physical properties of these particles in their size-quantised states have been well studied, there is relatively little information on the electrochemical and bioelectrocatalytic properties of these types of semiconductors. Even though some research has been conducted to predict the redox potential of these particles using the "particle-ina-box" model, there is not much clear proof concerning QDs due to technical challenges related to the limited solvent choices available to keep the particles in a stable form. One of the first pieces of evidence of redox activity of QDs was shown by Bard et. al. In this initial study, a direct correlation was found between the electrochemical band gap and the electronic spectra of QDs in organic medium. After this fundamental study, the impact of QDs started to be seen in the area of bioelectronics. One of the initial studies was conducted by hybridising $\mathrm{CdS}$ QDs with acetylcholine esterase (AChE) on an electrode surface. In this study, the product generated by the biocatalytic process activates the function of the QDs. The acetylcholine substrate is hydrolysed by enzyme on the particle surface and the hydrolysis products acts as an electron donor for the valence-band holes. As a result, the scavenging of the valence-band holes facilitates accumulation of the electrons in the conduction band of the electrode together with a generated photocurrent. In this way, a direct relationship between photocurrent signal and the substrate concentration was established. ${ }^{82}$

\subsubsection{Two-dimensional (2D) Materials}

Two-dimensional materials are usually defined as atomically thin, layered crystalline materials consisting of single layer of atoms. ${ }^{83,84}$ These materials including graphene and graphene derivatives, transition metal dichalcogenides such as tungsten disulphide $\left(\mathrm{WS}_{2}\right)$ and molybdenum disulphide $\left(\mathrm{MoS}_{2}\right)$, Xenes such as silicone, germanene, and diatomic hexagonal boron nitride (h-BN), have brought new perspectives and prospects in many different fundamental research areas from photonics to electrochemistry and their related fields of research including energy storage and production, and flexible (bio)electronic systems (Figure 14) ${ }^{85,86}$ These materials have recently attracted increasing attention due to their so called "extraordinary" physical and chemical properties, even though these properties have not been clearly defined yet. Because of these reasons, understanding the fundamentals of 2D materials in the aforementioned areas should be, in my opinion, the first focus rather than nondescript and poorly-described applications. Since the fundamental electrochemistry plays a key role in many areas such as energy storage and generation, and bioelectronics, we rather prefer to 
start this section of this chapter with an understanding of the basic physical, chemical and electrochemical principles of 2D materials. ${ }^{87}$ Here, we tried to limit two-dimensional materials to two-categories, even though they are more than that. Initially, we will try to explore the electrochemical properties of graphene and its derivatives by focusing mainly on graphene and following on from that, semiconducting transition metal dichalcogenides, by giving special focus only to $\mathrm{WS}_{2}$ and $\mathrm{MoS}_{2}$.

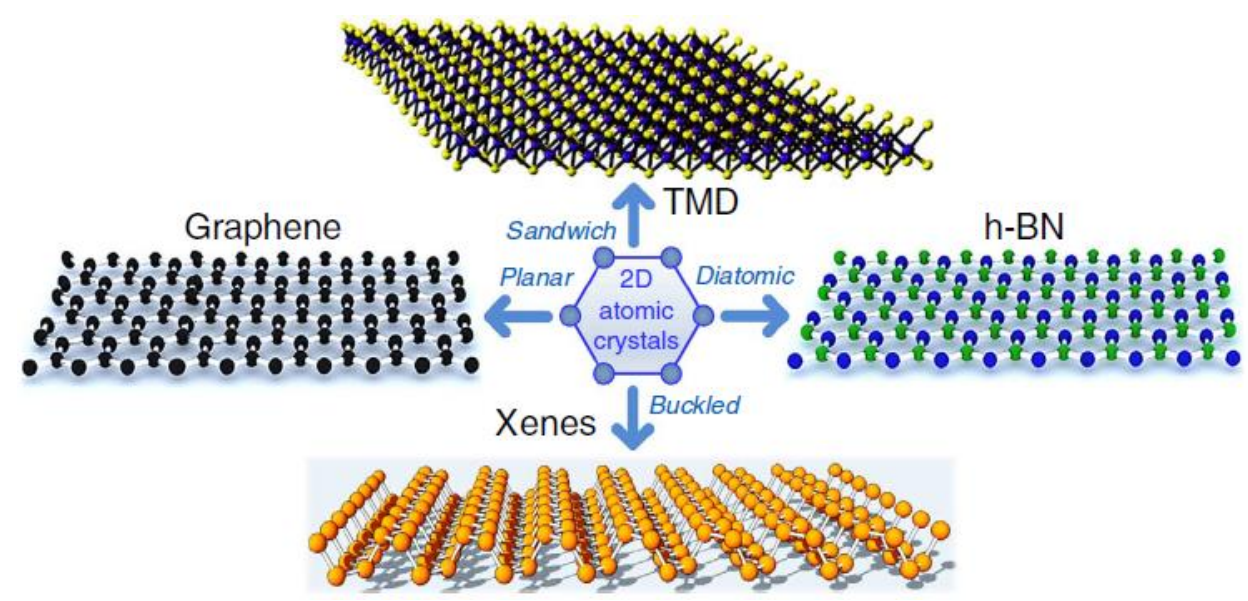

Figure 14 Schematic illustrations of various 2D materials. Copyright (C) 2014, Rights Managed by Nature Publishing Group. ${ }^{85}$

\section{Graphene and graphene derivatives}

Graphene consists of single layer of $\mathrm{sp}^{2}$-bonded carbon atoms arranged in twodimensional structure. It has "exploded" into many different research areas as a "promising" material, which brings exclusive chemical and physical properties. ${ }^{86}$ However, early discussions and theoretical explanations of graphene date back to the early $40 \mathrm{~s}$ and the proof of its existence as a single-layer material since the 60s. Recent interest on graphene has resulted mainly from the award of the 2010 Nobel Physics Prize given to Andre Geim and Konstantin Novoselov for their "ground-breaking experiments regarding to the two-dimensional material graphene" related to their very simple methodology with the so-called "scotch-tape" experiment in $2004{ }^{88}$ Even though graphene had been introduced before 2004 by other scientists, they generally failed to explain any of the "extraordinary" properties of graphene. Since 2004, and especially after the 2010 Nobel Prize, studies related to graphene and its derivatives and their applications have increased immensely. ${ }^{89}$ 


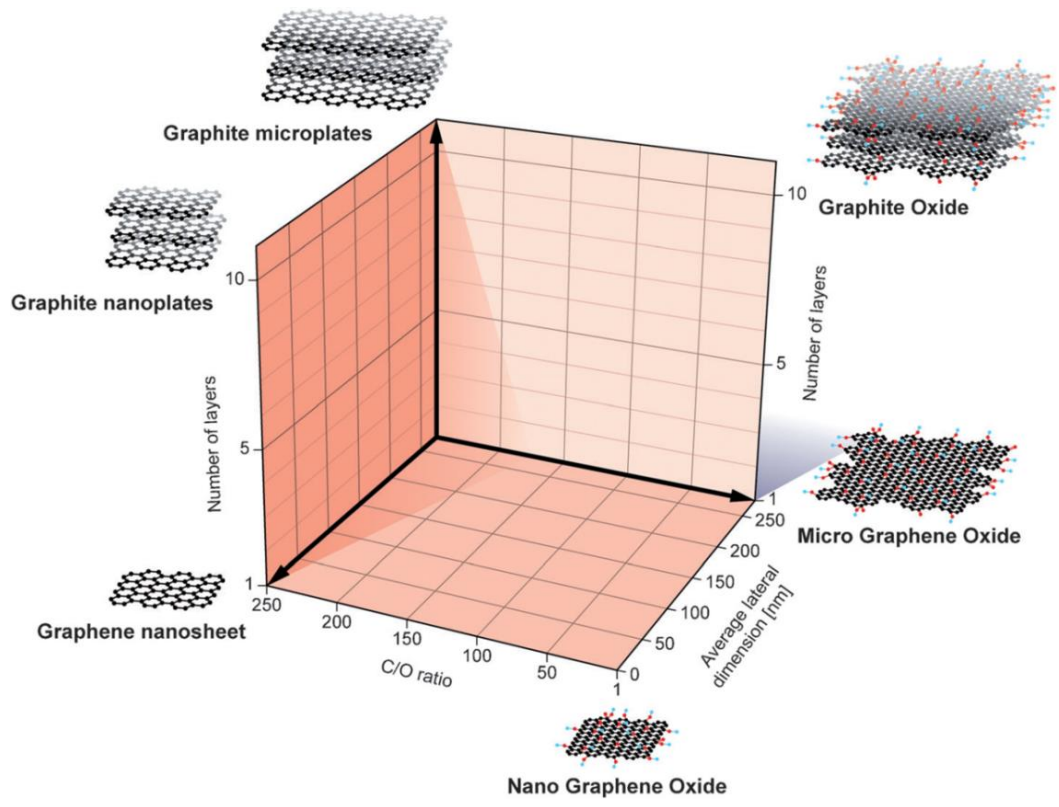

Figure 15 Classification of graphene-based materials. Copyright (C) 2014 WILEY-VCH Verlag GmbH \& Co. KGaA, Weinheim. ${ }^{90}$

Graphene is basically considered as a fundamental building block of similar types of carbon materials, including three-dimensional graphite, one-dimensional carbon nanotubes (CNTs) and zero-dimensional fullerene. In addition to its structurally similar materials, there are other types of atomically-thick, layered structure that are extensively used such as graphene oxide. Before going through the details about graphene, it is useful to understand the structural and chemical limits of the definition of graphene. Figure 15 shows a classification of different graphene types according to three fundamental graphene based material properties: number of layers, lateral dimensions and atomic carbon/oxygen ratio. ${ }^{90}$

Several synthetic methods have been developed recently for the synthesis of graphene. These methods are divided into two main categories in a similar fashion to the general methodologies for most nanostructure materials: top-down and bottom-up approaches as listed in Figure 16. ${ }^{86}$ 


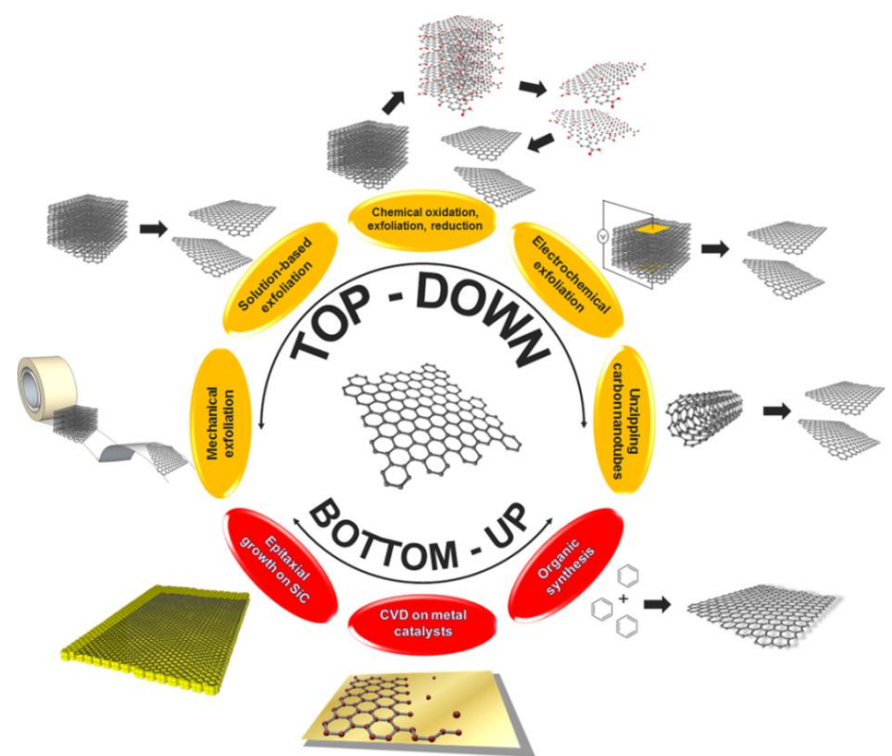

Figure 16 Schematic illustrations of synthetic approaches for graphene synthesis. Copyright (C) 2015 American Chemical Society. ${ }^{86}$

Graphene and graphene-based materials provide functional interface materials in the area of bioelectronics due to their various physicochemical properties, which allow control of their inter-facial electrochemical properties. ${ }^{91,92}$ Graphene and graphene-based materials are used mainly as voltammetric, potentiometric and impedimetric bio-sensors, and as field-effect transistor-based (bio)sensors due not only to their "unique" electrochemical properties, but also because of their ease of of processing and high-loading capacity of biomolecules together with biocompatibility.

Since graphene is the key building block for graphitic structures, the study of highly ordered pyrolytic graphite (HOPG) which has been conducted earlier gives very valuable information for today's graphene research. As described before, graphitic materials show anisotropic behaviour over different parts of same surface. ${ }^{88}$ The graphite surface possesses two distinctive parts having different electrochemical properties. These are called basal and edge planes. As depicted in Figure 17 and related studies, the electrochemical reactions on the edge plane are enormously faster than basal plane side, which is mainly due to structural defects on the former, even though the basal plane shows time-dependent chemical activity in some studies. ${ }^{89}$ Another important parameter for an electrode material and their electrical properties is the Density Of electronic State (DOS) which varies in different electrode materials. The DOS value of gold reaches up to 0.28 states atom ${ }^{-1} \mathrm{eV}^{-1}$, and the minimum reported DOS value of HOPG is 0.0022 
states atom ${ }^{-1} \mathrm{eV}^{-1}$, which is about $0.8 \%$ of gold. Hover, DOS value can be increased by introducing defects into the structure.

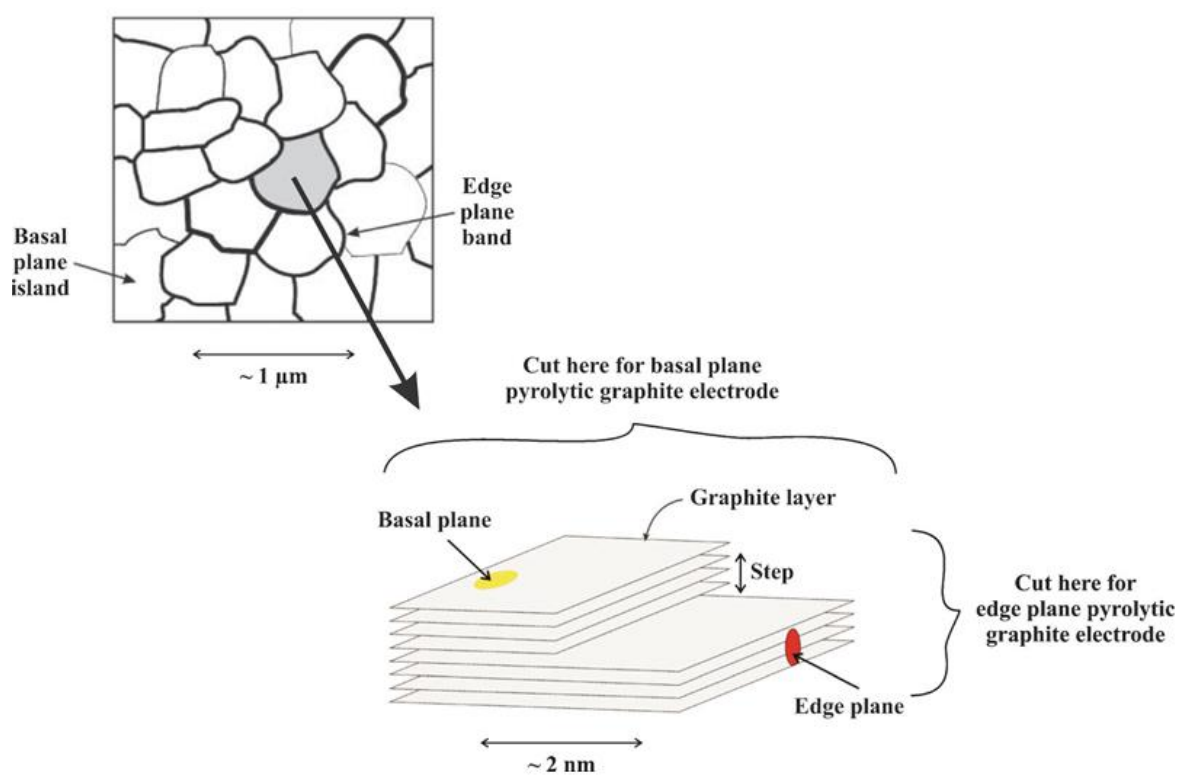

Figure 17 Schematic representation of a graphitic surface showing basal and edge planes. Copyright (C) Royal Society of Chemistry $2015 .^{88}$

One general way of using graphene as an electrode material is direct immobilisation on a macro-electrode surface such as gold or glassy carbon. However, it is important to note that immobilisation of graphene sheets on macroelectrodes generates a heterogeneous electrode interface. This creates complications in revealing the genuine properties of the graphene and usually results in misinterpretations. Under these circumstances, the underlying electrode material makes the main contribution or even dominates with respect to the general electrochemical activity of interface. The direct use of graphene without an underlying electrode surface, however, gives clearer information about the electrochemistry of graphene. ${ }^{93}$ Such results demonstrate that incorporation of individual monolayer crystals as an electrode material produces an ultra-microelectrode response. In this study, it was observed that the standard electrochemical rate constant was reduced for a ferrocene methanol $(\mathrm{FcMeOH})$ probe to $\sim 0.5 \mathrm{~cm} \mathrm{~s}^{-1}$. This value shows that the electrode material possesses faster electron transfer kinetics. The reason for this improvement in electron transfer kinetics is explained by edge plane-like defects across the basal plane of the graphene and exposed edges, which behave like ultra-microelectrodes to produce a sigmoidal response. ${ }^{94}$ All 
these properties make graphene an ideal interface material for electrochemical studies especially in the area of bioelectronics, where electron transfer and charge-transport are relatively difficult to achieve compared to other systems. ${ }^{88}$ Hence, the future of graphene in the area of bioelectronics is rather promising and offers considerable potential for the realisation of cutting-edge technologies, providing it is used in the correct way.

\section{Transition metal dichalcogenide (TMD) nanosheets}

Intensive research on two-dimensional materials, mainly focusing on graphene and graphene-based materials, has opened up new perspectives and discussions. ${ }^{95}$ However, graphene has failed in some ways to fulfil the requirements necessary for some specific applications, such as performing electronic switching, due to its lack of a natural energy band-gap. Recently, this shortcoming has been overcome using other types of two-dimensional materials as depicted in Figure 14.

Table 2.2 Electronic character of different layered transition metal dichalcogenides. Copyright (C) 2013, Rights Managed by Nature Publishing Group. ${ }^{96}$

\begin{tabular}{|c|c|c|c|}
\hline Group & $\mathbf{M}$ & $\mathbf{X}$ & Properties \\
\hline 4 & $\begin{array}{l}\mathrm{Ti}, \mathrm{Hf}, \\
\mathrm{Zr}\end{array}$ & $\mathrm{S}, \mathrm{Se}, \mathrm{Te}$ & Semiconducting $\left(\mathrm{E}_{\mathrm{g}}=0.2-2 \mathrm{eV}\right)$, diamagnetic \\
\hline 5 & $\begin{array}{l}\mathrm{V}, \quad \mathrm{Nb}, \\
\mathrm{Ta}\end{array}$ & $\mathrm{S}, \mathrm{Se}, \mathrm{Te}$ & $\begin{array}{l}\text { Semi-metals, superconducting, paramagnetic, an- } \\
\text { tiferromagnetic, or diamagnetic }\end{array}$ \\
\hline 6 & Mo, W & $\mathrm{S}, \mathrm{Se}, \mathrm{Te}$ & $\begin{array}{l}\text { Sulfides and selenides are semiconducting }\left(\mathrm{E}_{\mathrm{g}} \sim 1\right. \\
\mathrm{eV}) \text {, tellurides are semi-metallic, diamagnetic }\end{array}$ \\
\hline 7 & $\mathrm{Tc}, \operatorname{Re}$ & $\mathrm{S}, \mathrm{Se}, \mathrm{Te}$ & Small-gap semiconductors, diamagnetic \\
\hline 10 & $\mathrm{Pd}, \mathrm{Pt}$ & $\mathrm{S}, \mathrm{Se}, \mathrm{Te}$ & $\begin{array}{l}\text { Sulfides and selenides are semiconducting }\left(\mathrm{E}_{\mathrm{g}}=\right. \\
0.4 \mathrm{eV}) \text { and diamagnetic. Tellurides are metallic } \\
\text { and paramagnetic. } \mathrm{PdTe}_{2} \text { is superconducting }\end{array}$ \\
\hline
\end{tabular}

Transition metal dichalcogenides (TMDs) show quite different physical and chemical properties depending on their composition. For example, while some TMDs such as $\mathrm{HfS}_{2}$ exhibit insulating properties, $\mathrm{MoS}_{2}$ and $\mathrm{WS}_{2}$ show semiconducting behaviour, while $\mathrm{WTe}_{2}$ AND $\mathrm{TiSe}_{2}$ act like semi-metals. ${ }^{96}$ Similarly to the graphitic structures, TMDs also show anisotropic behaviour in their electrical, chemical and thermal properties. For example some TMDs, such as those based on Mo, W, Nb or Hf, have layered structures, while others like Pd or Ir 
exhibit non-layered structures. In addition, the stability of these layered structures results from the lone-pair electron of the chalcogenide atoms, which are able to terminate the surface of the layers and lack dangling bonds. ${ }^{11,84}{ }^{97}$ The electronic and magnetic properties of TMDs are summarised in Table $\mathbf{2 . 2}$
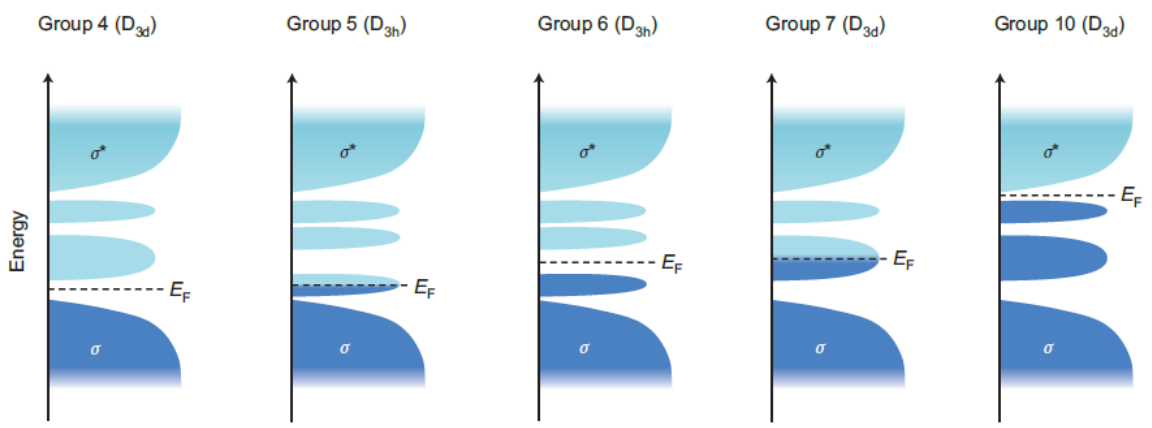

Figure 18 Gradual filling of the non-bonding d-orbitals that are located within the bandgap of bonding and antibonding states from group 4 to 7 and 10 TMDs. Copyright $\left(\right.$ C 2013, Rights Managed by Nature Publishing Group. ${ }^{96}$

The range of electronic properties listed in Table 2.2 arises from gradual filling of the non-bonding $d$ orbitals from group 4 to group 10. However, the effect of chalcogenide atoms on the electronic structure is not as strong as metal atoms, but there is also a trend that can be observed between them as shown in Figure 18. $^{96}$ 


\section{SWITCHABLE BIOELECTRONICS}

Switchable bio-interfaces having the ability to respond to real-life physical and chemical changes in their surroundings, have been commonly used in many different area of science and technology ranging from environmental studies to bioelectronics. ${ }^{1}$ The ability to control and modulate interfacial chemical and physical properties is crucial in most of these areas. Various materials have been used to create such interfaces and different characterisation methods have been developed to understand the interfacial properties of switchable interfaces. ${ }^{98}$ This chapter focuses on the general concept of switchable systems and characterisation methods (Section 3.1 and Section 3.2.). Section 3.3 and Section 3.4 give more specific examples of physically and chemically-stimulated switchable systems, respectively. In section 3.5, the recently emerging field of programmable bio-catalytic systems, particularly bio-interfaces is discussed. This chapter also covers topics which were not encompassed in our recently published review article, paper IV. More detailed information about switchable bioelectronics together with an up-to-date overview of its multifarious applications can be found in this paper (IV).

\subsection{Switchable Bio-interfaces}

Switchable interfaces are defined as systems having a responsive character which are able to provide a dynamic response to physical and chemical changes in their surroundings. Switchable interfaces are able to change their microscopic properties on demand and rearrange their interfacial properties. ${ }^{99}$

These changes are basically triggered using two different approaches: applying physical or chemical stimuli. Physical stimuli include change in surrounding temperature or light irradiation and change in magnetic and electrical field strength. Chemical stimuli are usually applied by changing the $\mathrm{pH}$ of reaction medium or indirectly by adding secondary molecules, which are able to change the $\mathrm{pH}$. Also, adding metals or salts and changing the ionic strength that can be used to affect the surface morphology. ${ }^{100}$

Switchable interfaces are usually constructed on a surface using two main approaches: self-assembled monolayers (SAM) and polymeric films. SAMs are introduced on a solid surface to generate self-organised and adaptive interfaces for further use. There are many different available features of SAMs on a surface, and they can be adapted to fit the objective of second step; head-groups 
such as thiols and silanes, for example, can bind strongly to the surface without functional end-groups, or molecules with reactive end-groups can be bound to create available interfaces for further immobilisations. These features of SAMs allow us to tune thickness, structure and surface energy of the interface by simply introducing different types of monolayers and additional modification of SAMs. Even though SAM technology was developed long time ago, switchable SAMs has been studied very recently to overcome the static features of conventional SAMs. ${ }^{101}$
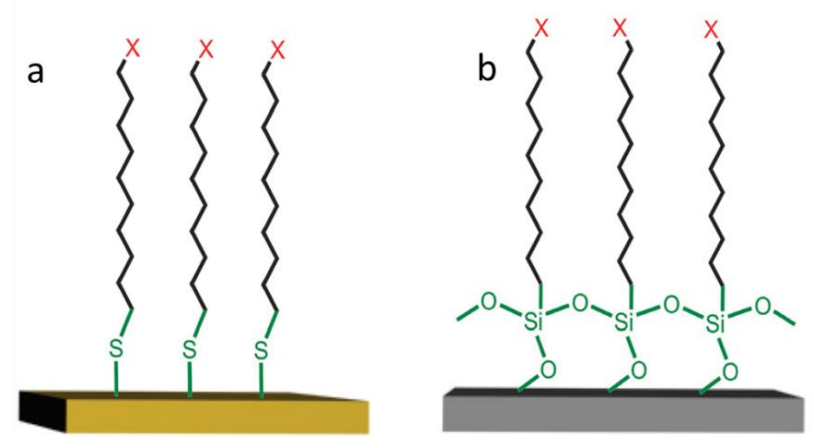

Figure 3.1 Schematic illustration of alkanethiol- (a) and alkanesilane-modified gold and silicon oxide surfaces, respectively. Copyright (C) Royal Society of Chemistry $2015 .^{101}$

Another general method to generate switchable interfaces is to use stimuliresponsive polymers. ${ }^{102}$ Even though stimuli-responsive polymer technology has been used for long time in drug-delivery and coating technology, it has only recently been employed in wider areas such as bioelectronics, to generate switchable devices, and microfluidics, to fabricate programmable devices. ${ }^{103}$ There are many different approaches available to create responsive polymeric films on solid supports. The methods include spin-coating, chemical vapour deposition, laser ablation and chemical and electrochemical reactions. Despite the fact that spincoating is considered as a relatively simple and practical method to generate polymeric film on solid surfaces, it suffers from some technical difficulties such as producing a large amount of waste material and rather thin-films when less soluble polymers are used. ${ }^{104}$ The other techniques such as laser ablation and vapour deposition are less practical. The electrochemical technique is also relatively easy, however it may result in problems and mismatches after forming the polymer film on the surface due to incompatibility with the electrolytes and sensitivity to different electrochemical conditions such as temperature and electrical stimuli (galvanostatic, potentiostatic). ${ }^{101}$ It is also impossible to grow polymeric 
films on an insulating surface via the electrochemical polymerisation method. One straight-forward method to grow robust and controlled polymeric films on solid supports is to use surface-tethered polymers, also known as polymer brushes. Polymer brushes are constructed by tethering of individual long-chain polymer molecules to specific anchor sites on the surface. In this way, a densely packed polymeric surface can be obtained and the random-walk conformation/motion of polymer that can occur in polymer solution or solution casted polymer can be restricted. Polymer brushes can be obtained either by physical or chemical methods, but chemical covalent linkage is preferred due to the stability it provides. There are two main approaches available for forming polymer brushes on a solid surface (Figure 3.2). ${ }^{101}$

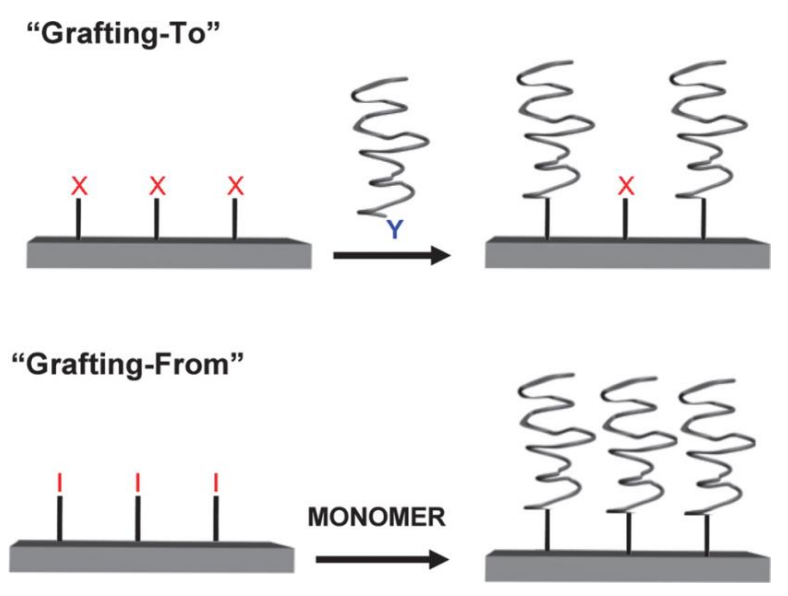

Figure 3.2 Schematic illustrations for the synthesis of polymer brushes using the "grafting-to" and "grafting from" approaches. Copyright (C) Royal Society of Chemistry 2015 . $^{101}$

In the "grafting to" approach, externally synthesised polymer molecules with different molecular weight are attached to the previously functionalised surface. However, in the "grafting from" technique, or so called surface initiated polymerisation, polymer is grown from surface via attachment of monomer units to the pre-functionalised surface. The grafting from approach provides more advantages over its grafting to counterpart. In the grafting from approach, a denser surface can be obtained because the grafting to approach usually suffers from steric hindrance after initial grafting of the polymer chains. Surface initiated polymerisation can be performed using many different polymerisation methods including free-radical, anionic, cationic, atom-transfer radical (ATRP), ringopening metathesis (ROMP), reversible addition-fragmentation transfer (RAFT) and nitroxide-mediated radical polymerisation (NMRP). ${ }^{105}$ 


\subsection{Characterisation of Switchable Surfaces}

The method of characterisation of switchable surface varies based on the switchable character of the surface. For different stimuli, there are different techniques to reveal changes such as change in morphology, wettability and permeability, and so on. ${ }^{100}$

One of the most common changes in switchable systems is hydrophobichydrophilic transition whether in SAMs or polymeric films. The change in surface wettability mainly results from reversible formation of hydrogen bonding in most of the system. Because of this, one of the most important tools to reveal change in the state of wettability is water-contact angle measurements. The water contact angle measurement provides a thermodynamic parameter to calculate the solid-liquid interfacial tension according to Young's equation: ${ }^{106}$

$$
\cos \Theta_{c}=\frac{\gamma_{s g}-\gamma_{s l}}{\gamma_{\mathrm{lg}}}
$$

In this equation, the contact angle $\Theta_{c}$, the liquid-vapour/gas interfacial tension $\gamma_{\mathrm{lg}}$, to the non-measurable interfacial tension $\gamma_{\mathrm{sg}}$ and $\gamma_{\mathrm{sl}}$ of the solid-vapour and solid-liquid interfaces can be obtained (Figure 3.3). The state of surface can be switched and directly measured upon applying chemical and physical stimuli. The application of this technique and detailed evaluation of water-contact angle measurements can be found in papers II, V and VI, VII.

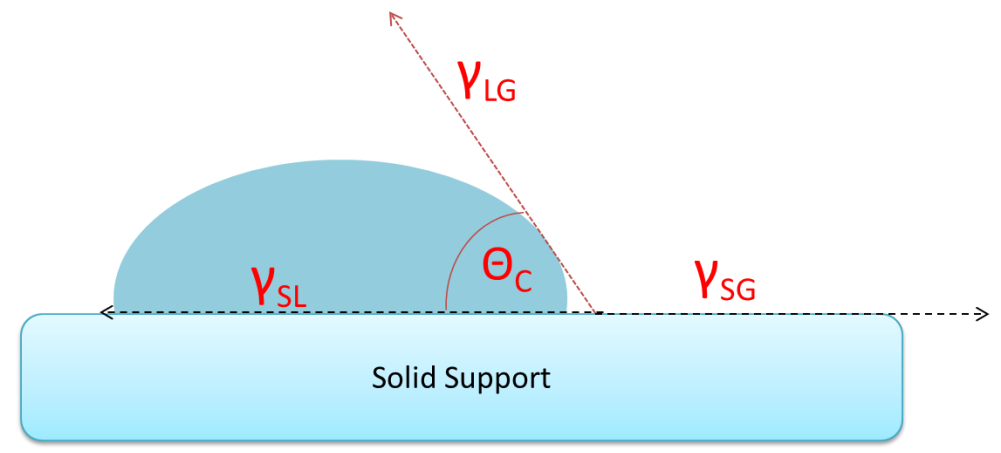

Figure 3.3 Schematic illustrations of the quantities in Young's equation.

Another distinctive change in switchable systems, especially in polymeric films, is the morphological change of the surface. Most of the polymer undergoes morphological transition (shrunk to swollen states) upon application of stimuli in accordance with wettability. The change in morphology brings various advantages to these systems such as controllable diffusion of species, conductivity, and permeability of the polymeric matrix. Because of this, study of the surface 
and related changes are extremely important. Microscopy techniques allow one to observe these changes. Optical microscopy provides in-situ and real-time observation of morphological changes in the surface as long as these changes occur in the macro-to-micro state. However, most changes occur in the nano-size range and because of that, more advanced microscopy techniques are required. These advanced microscopy techniques are usually electron microscopy methods such as scanning electron and transmission electron microscopies (Detailed explanation in Chapter 4). The drawbacks of these techniques include the necessity of measurement in the dry-state (usually all morphological changes occur in solution) and that results are not obtained in real time. Most of the measurements are carried out after freezing the samples in the two-different states and observing the difference under dry-state conditions. In one of our studies, we tried to overcome the technical challenges associated with observing morphological changes in real time. For this purpose, we designed an in-situ electrical-heating / water-cooling system for scanning electron microscopy. The specimens were directly heating on the SEM sample holder-stage in a dry-state (Paper V). This model system is only applicable and useful to observe changes on the surface as long as the morphology is dependent on temperature change. Morphological change resulting from light irradiation and magnetic or electrical field strength cannot be applied to this SEM set-up. However, in-situ electron microscopy techniques have been rapidly developing and real-time monitoring of any surface in any state may be revealed in parallel with the developments in electron microscopy. In addition to water-contact angle and microscopy techniques, electrochemical methods provide a unique opportunity to understand the switchable character of conductive surfaces. Using electrochemical techniques, wettability of the surface, conductivity and charge-transfer resistance can be quantified. The switchable interfacial properties of various surfaces using electrochemical techniques are described in detail in papers V, VI, VII, VIII.

\subsection{Physically-stimulated Systems}

\subsubsection{Light-switchable Interfaces}

Light is one of the most commonly used stimuli in the area of information processing. Optoelectronic devices have a non-invasive character, and offer spatial and temporal precision, but have some drawbacks such as low resilience, less reversibility etc. ${ }^{107}$ There are many different types of light-switchable systems that have been used to obtain light-responsive interfaces, such as azobenzenes, spiropyrans, fulgides, azulenes and many others, and they have been used in dif- 
ferent forms including as SAMs or by conjugation with polymer molecules or nanoparticles. ${ }^{108}$ Each light-responsive molecular system has specific mechanisms for their switching process, whether as a self-standing molecule or with conjugation. When some of the light-switchable molecules undergo reversible "trans-to-cis" isomerisation upon irradiation with specific light intensity, some of them show reversible redox-activity or sometimes they can undergo reversible heterocyclic bond cleavage upon irradiation. ${ }^{109}$

In our work, we have designed a light-switchable bio-interface (paper VII). In this study, the light-responsive methacrylated spiropyran (SPMA) molecular unit is co-polymerised with polyacrylamide (PAA) in order to yield a switchable bio-interface to control and regulate enzyme-based molecular interaction. Using electrochemical measurements, we have shown that interfacial bioelectrochemical properties can be controlled with a modest change in the wavelength of light. The system works based on designing a positively responding biointerface using a covalently attached polymer having a light-responsive polyacrylamide polymer co-polymerised with spiropyran (SP) molecular units and then conjugated with the graphene structure. In the polymer chain, SP units have two isomeric states, which are known as charge-separated merocyanine (MC) under UV light conditions, and spiropyran under visible light or dark conditions. The isomeric changes result in two important features of the interface; structural permeability and polarity differences. Here, these two states are reversibly controlled upon alternating UV and visible light. When the system is irradiated with UV light $(\leq 385$ $\mathrm{nm}$ ), SP undergoes a ring-opening conformation. This isomerisation results in volume and polarity changes. These changes are responsible for inducing permeability of the polymer structure (i.e. volume effect) and an increase in the conductivity (i.e. polarity effect). However, when the system is irradiated with higher wavelength light ( $\geq 420 \mathrm{~nm}$ ), the polymer structure turns to a densely packed form at the electrode interface, which results in lower permeability and diffusion of the substrate, and thus suppresses the electrochemical signals. As a result, the substrate cannot freely access the surface.

\subsubsection{Temperature-switchable Interfaces}

Another alternative stimulus to yield a switchable interface is temperature. SAMs or polymer films in the form of polymer-brushes, hydrogels or films generated by the layer-by-layer approach are commonly employed. The morphology and wettability changes of the structure on the surface, whether SAM or polymeric film, are mostly responsible for the behaviour of switchable interfaces, which allow control of the diffusibility of ions, analytes or redox species at the surface. ${ }^{1,110}$ 
Temperature switchable bio-interfaces are usually established to reversibly control molecular recognition events, cell attachment to solid surfaces or enzyme-based biocatalysis. The most commonly used polymer in these systems is poly(N-isopropylacrylamide-PNIPAAM). In paper $\mathrm{V}$, we used PNIPAAM together with a graphene-cholesterol oxidase hybrid to develop a switchable biointerface that is capable of positively responding. The system consists of a graphene as a donor and PNIPAAM as a receptor, assembled together based on donor-receptor interaction. At low temperature (i.e., at $20{ }^{\circ} \mathrm{C}$ ), hydrogen bonding interaction creates a coalescence on the surface, thereby causing considerable shrinkage in the donor-to-receptor interface. In this way, enzyme-substrate interaction is restricted and this results in a decrease in the diffusion of reactants and the consequent activity of the system. However, at high temperatures such as 40 ${ }^{\circ} \mathrm{C}$, the donor-receptor interaction is diminished and results in access of the substrate becoming easier to facilitate bioelectrocatalysis. ${ }^{91}$

\subsubsection{Electrically-switchable Interfaces}

Controlling interfacial physical and chemical properties of various surfaces using an electrical field provides a direct and quantitative way to design switchable (bio)interfaces which can be used in many different applications ranging from bioelectrocatalysis and marine biofouling to electrochromic windows. To design an electrically-switchable surface, the most common way is to use SAMs rather than a polymeric structure, due to the relatively fast-response of monolayer structures in the case of electrical switching. ${ }^{111}$

Electrical potential can be used as an external stimulus is to control cell adhesion during cell growth or reversible adsorption and release of protein onto electrically responsive structure-modified solid substrates. This method is important in various biological and medical applications including tissue engineering, cell-based drug screening and any other fundamental cellular studies. ${ }^{112}$ Most electrically-responsive systems use electroactive monolayers which are able to switch molecular activity. For example, silyl hydroquinone-modified Arg-GlyAsp tripeptide (RGD) is most used to form potential-switchable interfaces for cell adhesion. In this design, upon electrochemical oxidation of silylhydroquinone to benzoquinone, silyl ether is hydrolised and RGD peptide is released from surface at and applied potential of $550 \mathrm{mV}$. In this way, when the surface is treated with extra cellular matrix (ECM) protein fibronectin, a selectively prefered functionalized pattern is formed. ${ }^{101}$ 


\subsubsection{Magneto-switchable Interfaces}

One of the oldest methods known to generate a magnetically switchable interface is using magnetic materials. ${ }^{112}$ However, these interfaces can only be constructed by employing magnetic nanoparticles. Magnetic nanoparticles with different size, shape and surface functionalisation have been used in the area of magnetic resonance imagining (MRI), drug delivery, nano carriers and for separation of biomolecules. ${ }^{1}$ The most common way to use magnetic nanoparticles in the area of bioelectronics is to modify their surface with different redox-active molecules such as ferrocyanide or some ferrocene derivatives, to make use of their electrochemical mediating properties and control biomolecular interaction at the interface. $^{113}$ Following this approach, researchers have recently developed various power-on-demand biofuel cells and biosensor devices.

\subsection{Chemically-stimulated Systems}

Chemically-stimulated interfaces are usually formed by the addition or in-situ generation of some (bio)chemicals, by changing the reaction environment such as $\mathrm{pH}$ or ionic strength. These changes in the reaction medium affect surface morphology and wettability in a similar way to the physically-stimulated systems. ${ }^{114}$

The most common way to generate a chemically-switchable surface is to design a pH-responsive polymeric interface. These polymeric interfaces are formed by using layer-by-layer techniques, polymeric hydrogels or covalent and/or electrostatic attachment of redox species to a polymer matrix. ${ }^{115}$ There are two different approaches available to design $\mathrm{pH}$ responsive surface. ${ }^{114}$ In the first approach, the $\mathrm{pH}$ of the medium is changed directly by adding acidic or basic solution to control the interface. Another way is to design a system that is able to produce an in-situ $\mathrm{pH}$ change resulting from an enzymatic reaction. ${ }^{116}$ In most cases, researchers have exploited the electrochemical oxidation of glucose in the presence of glucose oxidase, which produces gluconic acid. The gluconic acid produced reduces the local $\mathrm{pH}$ and eventually affects the interface element, whether SAMs or polymeric structure. Apart from the effect of $\mathrm{pH}$ change on the reaction medium, addition of some molecule can affect the interfacial electrochemical properties. In one study, researcher developed a cross-linked porous polymeric film using polyvinylpyridine cross-linked with 1,4-diiodobutane (DIB) on the surface of an indium tin oxide (ITO) electrode. The designed polymeric interface exhibited reversibly opened and closed forms of the porous membrane in the absence and presence of cholesterol molecule. ${ }^{117}$ The presence of cholesterol results in formation of hydrogen bonds on the polymeric chain and swelling 
of the polymer, which closes the pores. When the polymeric is washed away, this hydrogen bonding is subverted and the system can return to the open formation. In this way, the charge-transfer resistance of the interface is controlled.

In paper VI, we have developed an interface that has an ability to respond to $\mathrm{pH}$ changes on the surface of an electrode for enzyme-based bio-catalysis using a pH-responsive poly(4-vinyl pyridine, P4VP) and glucose oxidase-modified graphene oxide. Here, P4VP polymer is used as a responsive element due to it having the ability to respond to $\mathrm{pH}$ change in the medium. This change results from protonation of the pyridine group in the polymer backbone under acidic conditions, which causes swelling of the polymer on the surface. This switchable swelling-shrinkage ability allows reversible diffusion of analyte through to the electrode surface. In our model system, we achieved control of enzyme-based biomolecular under two different $\mathrm{pH}$ conditions.

\subsection{Programmable Bioelectronics}

Even though designing of stimuli-responsive interfaces helps to control and regulate (bio)molecular interactions and eventually signal outputs, when the system involves many different inputs including different molecules or different reaction conditions, the overall system requires programming of each individual input and condition by segregating and/or putting all the parameters in order to maximise performance. However, current approaches are insufficient to satisfy these requirements. Recent studies, especially in the area of biotechnology, show that there is an obvious need to develop such systems with an ability to rearrange, control and order their functions. ${ }^{112}$ The programmable approach provides many opportunities to biotechnologists who specialise in artificial biological systems, such as mimicking living cells. It is well known that in nature, living reactions are naturally programmed, and attempts to construct artificial biological systems have generally resulted in vastly inferior performance. Because of this, in addition to advancement in the area of biotechnology to reach the target of artificial biological system, the idea of biocomputing and/or programming is necessary. ${ }^{118}$

\subsubsection{Enzyme-based Logic Systems for Biocomputing}

Initial work used enzyme as a simple biological element for the processing of enzyme-based biocatalytic reactions expressed in the language of information processing. ${ }^{119}$ Even though biocatalytic reactions are not really programmed, all interactions are defined in terms of programming language, which is crucial for initial steps. In this work, Boolean logic, which express the basic language of 
Boolean algebra using physical implementation of logic functions, was used to define biocomputing systems. For example, various Boolean logic operations such as AND, OR, XOR, NOR, NAND, INHIB, XNOR etc. are employed to produce suitable designs. ${ }^{14,120}$ For all these Boolean logic operations, the only allowed values are defined as 0 and 1.These logic operations as a description of Boolean function, are called logic gates. In each logic gate, logic 0 is represented by any output signal below a certain threshold level and logic 1 is referred to be above this threshold level. Many different biocatalytic processes have been expressed in terms of Boolean logic operation such as using couple enzyme in solution in an optical system or by interfacing of enzyme logic with signal responsive materials as an electrochemical system. ${ }^{121}$

One of the most realistic biochemical computing studies has been developed using enzyme-based logic gates interfaced with signal responsive materials. In this design, enzyme molecules are used as a biocatalytic input signal to process the information according to the Boolean functions AND/OR (Figure 3.4). Here, AND-gate design works based on a sequence of biocatalytic reactions starting with sucrose hydrolysis by invertase which produces glucose. The glucose produced is oxidised by oxygen in the presence of glucose oxidase. The later reaction results the formation of gluconic acid which helps to reduce the local $\mathrm{pH}$ of the interface as the output of the gate. In this design, the absence or presence of enzymes are considered as input signal 0 and 1, respectively. In the AND gate, the biocatalytic chain reaction can only be active in the presence of both enzymes; glucose oxidase and invertase $(1,1)$. The absence one of enzyme $(0,1$ or $1,0)$ or both of them $(0,0)$ results absence of the gluconic acid formation and the system does not produce a positive output signal. In addition, this system requires an additional enzyme (urease) to reset the $\mathrm{pH}$ of the medium to the original value after reaction with urea. In the OR gate design, the system works astwo parallel reactions. The hydrolysis of ethyl butyrate and oxidation of glucose are biocatalysed by esterase and glucose oxidase, and these reactions produce butyric acid and gluconic acid, respectively. The produced acid results in reduction of the local $\mathrm{pH}$ and generates the signal. In this design, the input signals are two enzymes; glucose oxidase and esterase. The presence of both enzymes are considered as $(1,1)$ and when either enzyme or both of them together are absent the system is considered as $(0,0)$ and $(0,1$ or 1,0$)$, respectively. In this study, the output signal is the $\mathrm{pH}$ of the medium, but it can be changed for different systems, such as optical or electrochemical, based on the same idea. 


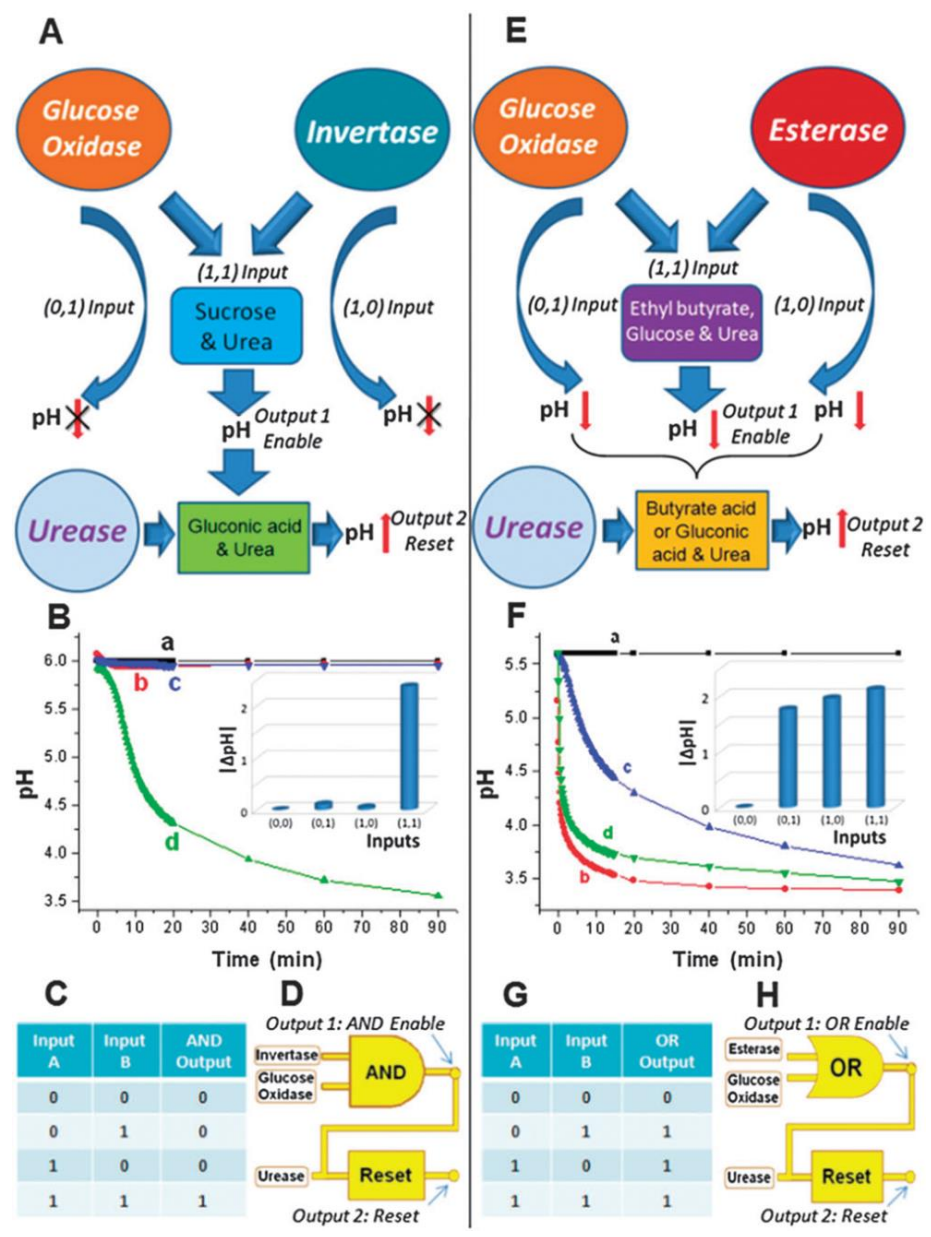

Figure 3.4 Schematic illustrations of output signal and truth table for AND gate (a-d) and OR gate designs (e-h), respectively. Copyright (C) Royal Society of Chemistry 2015. ${ }^{120}$

\subsubsection{Programmable Enzyme-based Biocatalytic Systems}

Even though the work presented in previous section and other similar works are considered as an initial work in biocomputing, they are still far from a programmable design. The reason is that the idea of presence or absence of enzyme in the biological system, is not realistic. If one attempts to design and mimic a biological system, the presence of every molecule and the other conditions ( $\mathrm{pH}$, temperature etc) should be considered in advance. The best way to program any biological system is to change the parameters without using interfering biomolecules 
(such as amount, types). The target should be to control the interaction between the biomolecules. For this purpose, we attempted to design a programmable biointerface to control and regulate enzyme-based biocatalytic reaction, segregate and eventually program their interactions by external physical stimuli. In paper VIII, we have presented, for the first time, a design for programmable interface with switchable and tuneable bio-catalytic performance that responds simultaneously to appropriate stimuli. The designed bio-electrodes are composed of light and temperature responsive compartments in different orientations, which allow us to build Boolean logic gates (OR and AND) based on enzymatic interactions to deliver logic operations. 


\section{EXPERIMENTAL METHODS}

\subsection{Polymerisation Methods}

Polymer researchers around world have expended considerable effort to better understand synthetic polymers and their structure-property relations by investigating new polymerisation techniques, introducing new and low cost monomers and trying to find a tailor-made concept for polymer synthesis. After a century of research, we are finally now ready to produce polymers from different sources with any quality as an end-product. Polymer materials can be produced in many different forms of plastic, fibres, elastomer or foam. They can be hard or soft, gels or films, conductive or insulating, inert or responsive, porous or nonporous etc. But all of these choices are possible only because of advances in different synthesis techniques in parallel with advancement in chemical sciences. ${ }^{122,123}$

Polymers are categorised in many different ways. The most popular categorisations are listed below. They can be classified according to their source of origin, such as natural or synthetic, or sometimes according to their structure, such as linear, cross-linked etc., or according to the synthesis technique such us chain-growth, step-growth, or ring opening, and finally according to their thermal properties, such as thermosets or thermoplastics. Here, in this section we categorise polymer according to their production method.

The polymers are classified into two main categories depending on their polymerisation mechanisms as chain-growth (addition), step-growth (condensation) and ring-opening polymerisations.

\subsubsection{Chain-growth Polymerisation}

Chain-growth polymerisation (addition polymerisation) is the most commonly employed technique both at an industrial scale and in academic studies due to its faster kinetics and relatively easy processing. ${ }^{124}$ The most-well know unsaturated molecules that can undergo addition polymerisation are the olefins which in its simplest form is vinyl in Figure 4.1. 


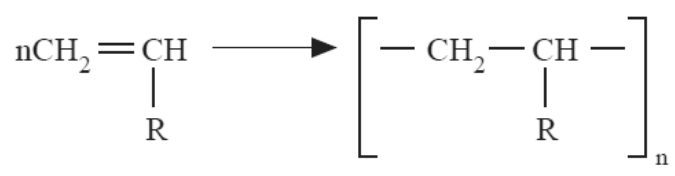

Figure 4.1 Chemical structure of typical vinyl monomer and its polymeric form.

The growing mechanism of addition polymerisation is based on radical formation, which grows via a chain mechanism. Chain-growth polymerisation contains several steps including initiation, propagation and termination.

Initiation starts by generation of the active site of monomers. This can occur via different ways such as heating, high-energy irradiation or just simply by irradiating with ultraviolet light. However, the most common way to generate the active site of a monomer to initiate the reaction is to use small molecules known as "initiators". Even if the initiator starts the polymerisation they are not considered as catalyst because they are chemically changed during the polymerisation process. The initiators are usually weak compounds which can easily decompose to form free radicals. The formation of the radical for initiators occurs at modest temperature, or low wavelength of visible light or near infrared radiation. Initiation principally consists of a two-step reaction. In the first step, the radicals are formed as described and in the second step, initiators are added to the vinyl monomer (Figure 4.2a). In second step, the initiated monomer starts to propagate, in other words, each monomer units starts to be chemically linked with each other and continues until the growing chain radical is deactivated by chain termination (Figure $4.2 \mathrm{~b}$ ). In the termination step, the growth activity of polymer chain radical is stopped by reaction with another free radical system in the polymerisation medium. There are two different termination mechanisms available, one is called coupling termination (Figure 4.2c) where two growing chains are coupled with each other, and the other is called disproportionation (Figure 4.2d), where a labile atom (usually hydrogen) is transferred to terminate the reaction. In the coupling process, only one polymer is produced, however the disproportionation process yields two polymeric chains. In addition to these three main steps, there is one more step that may be involved in the polymerisation process, which is known as chain transfer. In this step, an already growing polymer chain is terminated by transferring its activity to one of the previously inactive species. ${ }^{125}$ 


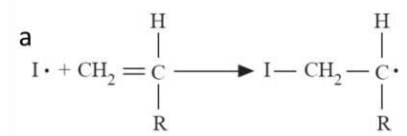<smiles>[R][CH+]CC=C[IH]CC([R])CC([R])[R]</smiles><smiles>[Y13]CC([R])C([R])CC[AlH]I</smiles>

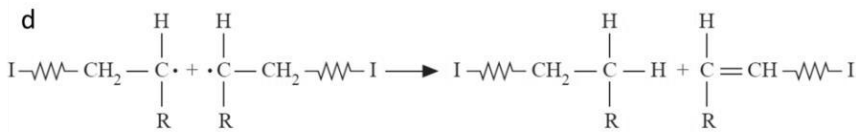

Figure 4.2 Illustration of steps in chain-growth polymerisation including initiation (a), propagation (b), and termination via coupling (c) and disproportionation (d).

In addition to the described mechanism based on free-radicals chain growth, polymerisation can be performed by cationic, anionic and coordination polymerisation following the same steps. But at each step free-radicals should exchange with cation and anions. There are many different types of subgroup of addition polymerisation including living radical polymerisation such as atom-transfer radical (ATRP), reversible addition-fragmentation chain-transfer polymerisation (RAFT) etc. In these types, polymer molecular weight can be controlled in contrast to the general free-radical polymerisation, which is described here in this section.

\subsubsection{Step-growth Polymerisation}

In step-growth (condensation) polymerisation, large molecules are formed by a series of reactions between two types of molecules, such as monomers, dimers and trimers. ${ }^{126}$ In this type of polymerisation, step-wise reactions of esterification, ester interchange and amidisation occur between pairs of molecules, and small molecules such as water are eliminated. In condensation polymerisation, the reaction occurs between one or more types of monomers, where each reactive building block possess two functional groups. Step-growth polymerisation is categorised into two main mechanisms: polycondensation, where a small molecule is eliminated at each step, or where monomers react without the elimination of a small molecule. 
Polymers are also categorised according to their composition, whether they are produced via addition or condensation reactions. If the polymer is composed of only one type of monomer in the final structure, it is known as a homo polymer. However, in order to increase the functionality and physical and chemical properties of one type of polymer, it is possible to synthesise it in such a way that the polymer can be composed of more than one type of monomer unit. These types of polymers are called as copolymers. The repeating units on the copolymer chain can be arranged in various degrees inside the backbone using special polymerisation techniques. There are many different types of copolymers are available as listed below. ${ }^{127}$

Random copolymer: In this type, each different monomer unit (A and B) are randomly rearranged in the polymeric backbone and they are shown as: ABBAB ABABBABABABAA-

Alternating copolymer: In this type, each monomer unit follows one other in specific order and it is demonstrated as: $-\mathrm{AB} A B \mathrm{AB} A B \mathrm{AB} A B \mathrm{AB} A B-$

Block co-polymer: In this type, each monomer unit is distributed along the chain in large and repeating sections, known as a block. The structure is simply shown as: -AAAA-BBBBBB-AAAA-BBBB-

Graft copolymer: In this type, the sequences of monomers are grown from a main backbone. They are simply shown illustrated:

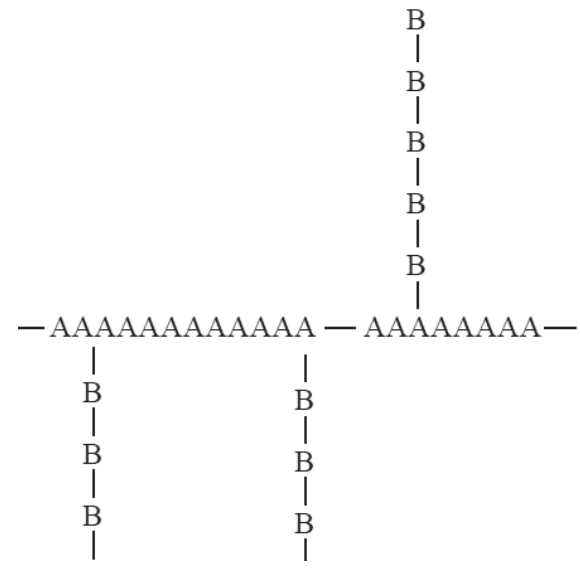




\subsection{Enzyme Immobilisation Methods}

One of the most important steps for the interfacing of nanomaterials with biomolecules is to provide for stable integration of biomaterials into the nanomaterial and eventually to the bioelectronic interface, to sustain their activity and performance. ${ }^{128}$ In the enzyme-based biocatalytic systems described in all the papers included in this thesis, the enzymes are immobilised on nanostructure surfaces to yield efficient bioelectronic devices. In all papers in this thesis, electrostatic immobilisation is preferred, however there are many different types of immobilisation available and they are listed in the section below.

\subsubsection{Adsorption}

In this approach, enzymes are linked to a surface via mainly electrostatic attraction and other weak forces such as those mentioned in Chapter 2. The main advantage of the adsorption technique is that it is a relatively easy and fast method rather compared to other approaches. However, it is also not as stable as other techniques in that the electrostatically adsorbed enzymes can easily leach out from the surface depending on the change of reaction environment such as $\mathrm{pH}$ or ionic strength. ${ }^{129,130}$

\subsubsection{Covalent Bonding}

In order to overcome the problem of stability in the adsorption method, covalent bonding between biomolecules and the surface is used as an alternative technique. However, during the process of covalent bonding, usually the catalytic activities of biomolecules are negatively affected due to the relatively harsh conditions. The most commonly employed type is 1-Ethyl-3-(3dimethylaminopropyl)carbodiimide (EDC) and $N$-hydroxysuccinimide (NHS) coupling that works well with most of the enzymes. Here, EDC can easily react with NHS and form an NHS-ester that allows conjugation to primary amines at of enzyme periphery. ${ }^{129}$

\subsubsection{Cross-linking}

Similarly to the covalent linkage of biomolecules to the surface, in cross-linking methods biomolecules are cross-linked with each other. Even though this technique provides a quite stable biomolecular matrix, it often affects badly the activ- 
ity of most biomaterials including enzymes. The most commonly used crosslinking compound is glutaraldehyde, which links enzymes by reaction with their primary amine structure. ${ }^{129,131}$

\subsubsection{Entrapment}

The last method is entrapment or encapsulation. In this method, biomolecules are entrapped inside the polymeric matrix, usually conjugated polymers, hydrogels or any commodity polymers. The disadvantage of this method is the difficulty of communication between the substrate and the enzyme, which is strongly hindered due the insulating interface. However, this method provides a flexible distance to the enzyme and helps to retain the guest's native structure. ${ }^{129,132}$

\subsection{Microscopy Techniques}

The microscopy techniques used are indispensable complementary techniques that help us to understand the morphological features of a surface by using a beam of electrons to reveal an image. ${ }^{133}$ They provide not only higher magnification, but also higher resolution than optical microscopes and allow us to observe smaller objects in comparatively better detail. Especially in the area of switchable bioelectronics, electron microscopy techniques are crucial to see the changes on the interface before and after applied stimuli. But before going through the detail of the specific techniques that are used in the papers, it might be good to briefly describe the theory of electron microscopy.

The high resolution of the electron microscope is mainly provided by the design of electromagnetic lenses. Instead of the glass lenses in an optical microscope, the electron microscope uses electromagnetic and electrostatic lenses to control the electron beam. The electromagnetic lenses are designed in the form of a solenoid, a coil of wire covering the outside of the tube, where electron beam passes through to the sample. When a current pass through the tube, an electromagnetic field is induced and the electron can be controlled by simply changing the current. As it is well known, increasing the current makes the electrons move faster and this produces shorter wavelengths. In electron microscopy, shorter wavelengths increase resolution. ${ }^{134}$ 


\subsubsection{Transmission Electron Microscopy (TEM)}

The TEM works according to a similar principle as optical microscopy, but instead of having photons TEM has electrons. TEM uses an electron gun instead of light bulb and has magnetic lenses instead of glass lenses. ${ }^{135}$ TEM uses an electron beam that can be transmitted through the very thin specimen to carry information about the structure of the sample. Then the image is magnified by a series of magnetic lenses until reflecting to the fluorescent screen, or light sensitive CCD (charge-coupled device) camera. Then detected image is shown in real time on a monitor or via a computer.

TEM is commonly the preferred technique and is frequently used in both industry and academia to investigate the microstructure and composition of any sample having thickness less than $100 \mathrm{~nm}$, so that electron beam can penetrate the sample. A resolution in the order of 1 Angstrom $(\AA)$ can be achieved under optimum conditions. ${ }^{135}$

In all papers in this thesis, TEM has been used with predefined parameters to reveal the structure of $2 \mathrm{D}$ graphene and $\mathrm{WS}_{2}$ crystals and assembling of nanoparticles and enzymes.

\subsubsection{Scanning Electron Microscopy (SEM)}

In SEM, the electrons are not transmitted through the sample, in contrast to the TEM technique. Here, the images are produced by detecting secondary electrons which are emitted from the surface excited by primary electrons. In SEM, the surface is scanned by an electron beam and the detector captures the image by mapping the detecting signal based on beam position. Even though the resolution of SEM is lower than the TEM, it has the advantage of revealing three dimensional images ${ }^{136}$

In almost all paper in this thesis, SEM has been used to observe the 3D structure of graphene, polymer and enzyme assembling structure to analyse the surface of an electrode material. Most importantly, in paper $\mathrm{V}$, which focuses on a temperature-controlled bioelectrocatalytic interface, we designed a specific experimental set-up to understand how the morphology of a temperature-responsive polymer surface changed upon direct heating of the surface. For this purpose, we used in-situ electrical heating-water cooling system that is integrated with SEM as shown in Figure 4.3. Here we directly heat the sample to $40{ }^{\circ} \mathrm{C}$ and tried to see the difference after change in temperature. ${ }^{91}$ 


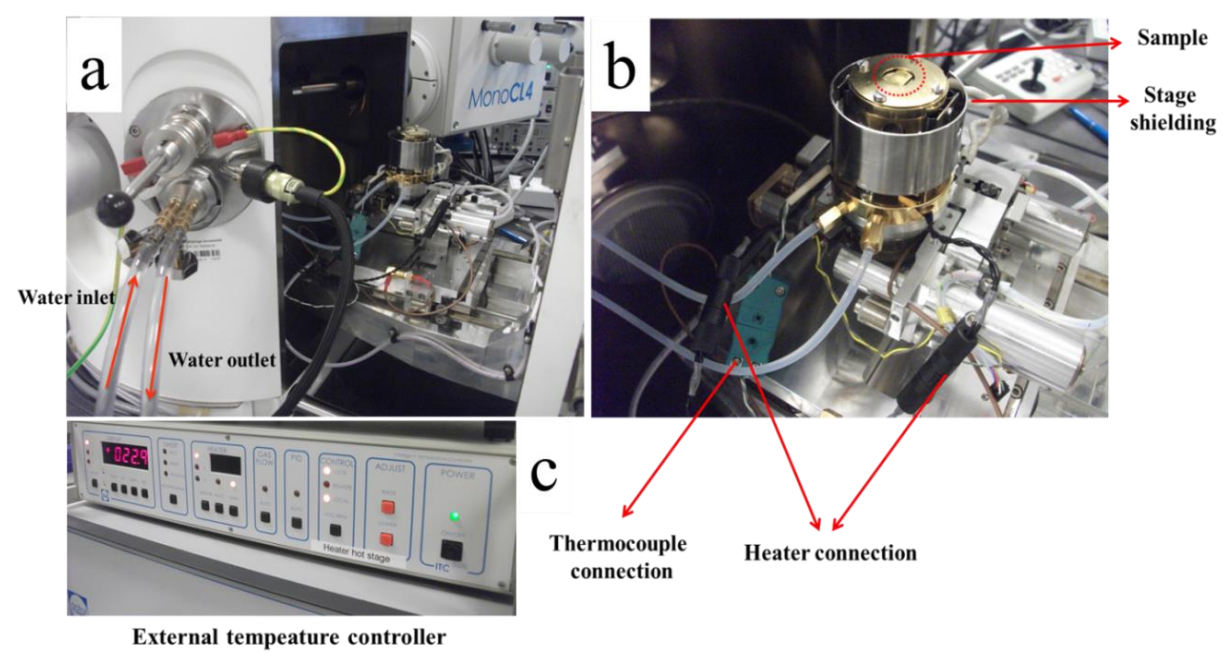

Figure 4.3 Set-up for temperature-controlled SEM experiments with lower (a) and higher (b) magnifications, and external temperature control device (c). Copyright $\left(2013\right.$ WILEY-VCH Verlag GmbH \& Co. KGaA, Weinheim. ${ }^{91}$

\subsection{Electrochemical Methods}

In this section, all electrochemical methods (voltammetry, amperometry and impedance spectroscopy) that were used in this thesis are introduced. For electrochemical measurements, a three-electrode configuration was used. The three electrodes were working electrodes such as gold plate $\left(0.5 \mathrm{~cm}^{2}\right)$ and glassy carbon electrode $\left(0.07 \mathrm{~cm}^{2}\right)$, platinum wire auxiliary and $\mathrm{Ag} / \mathrm{AgCl}(3 \mathrm{M} \mathrm{KCl})$ reference electrodes.

\subsubsection{Voltammetry}

Voltammetry is commonly employed for electroanalytical measurements. ${ }^{137}$ Voltammetric techniques are usually categorised into three groups as follows:

I. Potential step voltammetry

II. Linear sweep voltammetry

III. Cyclic voltammetry

Basically in each technique, a varying voltage is applied to the electrode and current is followed via different approaches. 


\section{Potential Sweep Voltammetry}

In the potential step technique, the voltage that is applied to the electrode is elevated from one value $\left(\mathrm{V}_{1}\right)$ to the other $\left(\mathrm{V}_{2}\right)$ and current change is measured as a function of time. ${ }^{138}$ A typical illustration of potential step voltammetry is as follows (Figure 4.4).
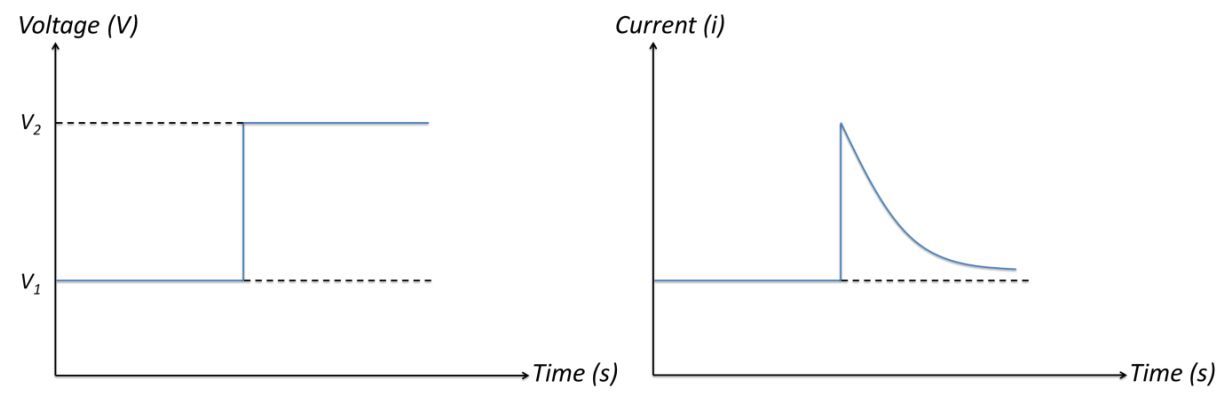

Figure 4.4 Schematic illustration of potential step (left) corresponding current response (right) in potential step voltammetry.

When the voltage is fixed at low values $\left(\mathrm{V}_{1}\right)$, the reduction of reactant $\left(\mathrm{X}^{3+}\right)$ might be unfavourable, however when the voltage is increased to some specific value $\left(\mathrm{V}_{2}\right)$, the reactants close to the electrode surface might be reduced to the $\mathrm{X}^{2+}$ and the current can change as depicted in Figure 4.4 (right).

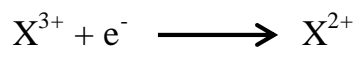

Here, when the voltage is increased suddenly the current rises and then starts to drop back as a function of time, due to the slow diffusion of reactant to the electrode surface. As the concentration decreases therefore, the current also drops over time.

\section{Linear sweep voltammetry}

In the linear sweep technique, potential is applied over a certain range, but unlike in the potential step technique, potential is scanned from lower to the higher value. ${ }^{138}$ Here, current change is recorded as function of sweep voltage rather than a time, based on the same reaction is shown in equation 4.1. At low applied voltage there is no net current flow on the current-voltage plot in Figure 4.5. However, when the voltage is swept through to higher value more reduction results more current flows. Another important point is the effects of scan rate on peak current. 
At slow scan rates, the diffusion layer grows slowly, but extends further from the surface of electrode than at a faster scan rates. As a result, flux to electrode surface becomes smaller in this range. ${ }^{137}$ Due to the direct relationship between current and flux through the electrode surface, the peak current becomes lower at low scan rates and higher at high scan rates.
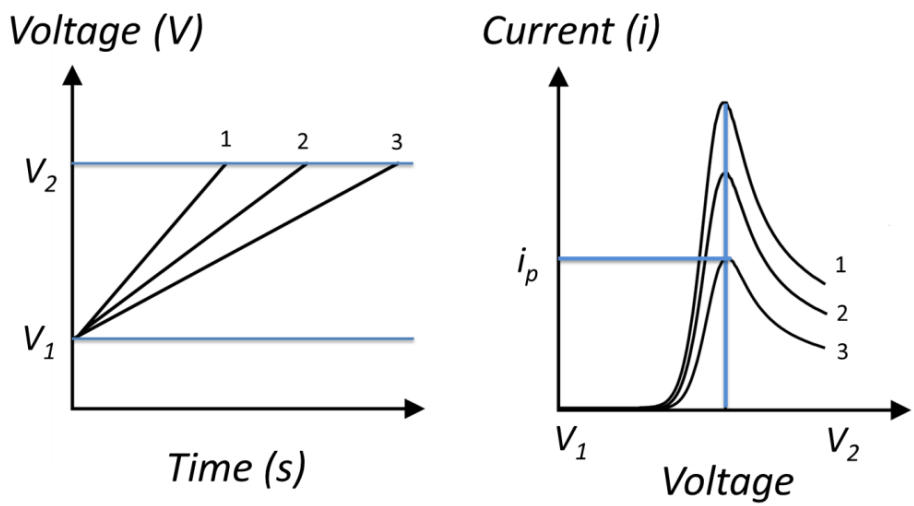

Figure 4.5 Schematic illustration potential sweep (left) and corresponding current response (right) in potential sweep voltammetry.

\section{Cyclic voltammetry}

The only difference between cyclic voltammetry and linear sweep voltammetry is that the potential is scanned at fixed scan rate and is reversed between two voltages values in cyclic voltammetry. ${ }^{138}$ Similar to the linear sweep technique, changing of scan rate has the same effect and explained for a reversible electron transfer reaction in terms of the diffusion layer thickness. For reversible electrode reactions, peak currents are proportional to the square root of the scan rates. 


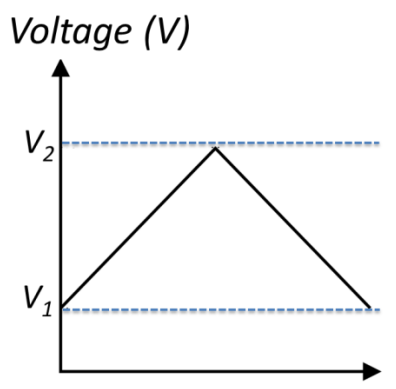

Time (s)
Current (i)

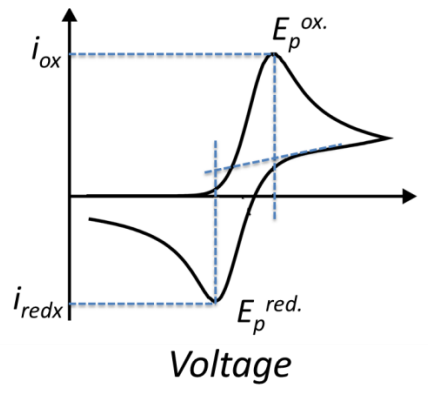

Figure 4.6 Schematic illustration potential sweep (left) and corresponding current response (right) in cyclic voltammetry.

The voltage separation between the current peaks is defined as follow;

$$
\Delta E=E_{p}^{o x}-E_{p}^{r e d}=\frac{59}{n} m V
$$

Where $\mathrm{n}$ is number electron transferred and $\mathrm{E}_{\mathrm{p}}{ }^{\mathrm{ox}}$ and $\mathrm{E}_{\mathrm{p}}{ }^{\text {red }}$ are defined as oxidation and reduction potential, respectively. In reversible electrode reactions the position of peak voltages does vary based on the scan rate values. ${ }^{137}$

\subsubsection{Amperometry}

Amperometric methods, especially chronoamperometry have been extensively used in this thesis to investigate the performance of electrochemical biosensors. ${ }^{137}$

\section{Potential step amperometry}

In chronoamperometry, the current is monitored as a function of time in response to a potential pulse. In the initial step, the applied voltage of an electrode is kept constant $\left(\mathrm{V}_{1}\right)$ and then instantaneously raised to a new value $\left(\mathrm{V}_{2}\right)$ and the corresponding current as a function of time is recorded, as depicted in Figure 4.7. 


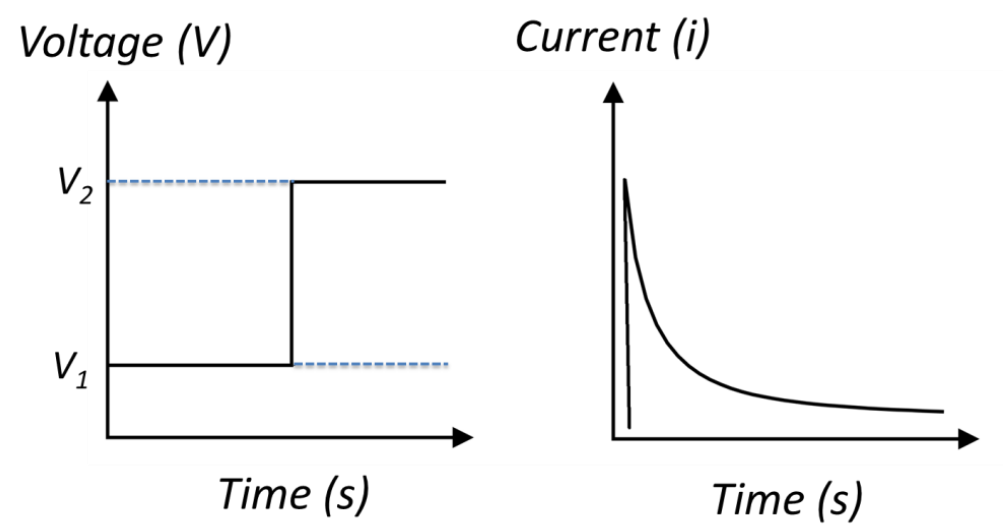

Figure 4.7 Schematic illustration applied potential (left) and corresponding current response (right) in potential step amperometry.

It is important to note that such measurement should be made over a relatively longer time in order to be sure about the system, because short-term experiments cannot exclude or differentiate the current resulting from large non-faradaic events such as charging double-layer capacitance. ${ }^{139}$

\subsubsection{Impedance Spectroscopy}

Impedance spectroscopy or dielectric spectroscopy basically relies on the concept of electrical resistance. It is well known that every circuit element resists the flow of electrical current based on Ohm's law, which defines the resistance based on a relationship between voltage and current. Here, the resistance value is independent of frequency; the current and voltage signal are in phase together. ${ }^{137}$

However, when different circuit elements are involved the process, the concept of resistance is replaced with impedance. Similar to resistance, impedance also allow us measure resistance to electrical current flow, but electrochemical impedance is measured by reading out the current signal by the application of a sinusoidal AC potential to an electrochemical cell. The impedance is expressed as a complex number as follows: ${ }^{137}$

$$
Z(\omega)=\frac{E}{I}=\frac{E_{0} \sin (\omega t)}{I_{0} \sin (\omega t+\phi)}=Z_{0} \frac{\sin (\omega t)}{\sin (\omega t+\phi)}
$$

where $\mathrm{Z}, \omega$ and $\phi$ define impedance, radial frequency and phase shift, respectively. The electron transfer properties of an electrode can be quantified by using electrochemical impedance spectroscopy. Usually, one of the simple models such as Randles circuit is chosen to fit the impedance outputs to calculate the charge transfer resistance. In addition, in most impedance results introduced in this the- 
sis, a Nyquist plot is chosen. The Nyquist plot contains semi-circular and linear portions. The plot indicates both electron transfer limited (semi-circular portion) and diffusion processes (linear portion) occurring at the same time. The charge transport resistance $\left(R_{\mathrm{CT}}\right)$ at the electrode surface can be quantified based on the diameter of the semi-circular part of plot and described by following equation: ${ }^{137}$

$$
Z_{(\omega)}=R_{s}+\frac{R_{C T}+\sigma \omega^{-1 / 2}}{\left(C_{d} \sigma \omega^{1 / 2}+1\right)^{2}+\omega^{2} C_{d}^{2}\left(R_{c T}+\sigma \omega^{-1 / 2}\right)^{2}}-j \frac{\omega C_{d}\left(R_{C T}+\sigma^{-1 / 2}\right)^{2}+\sigma \omega^{-1 / 2}\left(\sigma \omega^{1 / 2} C_{d}+1\right)}{\left(C_{d} \sigma \omega^{1 / 2}+1\right)^{2}+\omega^{2} C_{d}^{2}\left(R_{C T}+\sigma \omega^{-1 / 2}\right)^{2}} \quad 4.4
$$

where $R_{\mathrm{s}}$ is the solution resistance, $C_{\mathrm{d}}$ is the double layer capacitance, $\omega$ is the $2 \pi f$, where $\mathrm{f}$ is the frequency, and $\sigma$ is defined as:

$$
\sigma=\frac{R T}{\sqrt{2} F^{2} A}\left(\frac{1}{\sqrt{D_{o}} C_{o}^{*}}+\frac{1}{\sqrt{D_{R}} C_{R}^{*}}\right)
$$

where $\mathrm{A}$ is the area of the electrode, $D_{o}$ and $D_{R}$ are the diffusion coefficients of oxidant and reductant, respectively. $\mathrm{C}_{\mathrm{o}}$ and $\mathrm{C}_{\mathrm{R}}$ represent the bulk concentrations oxidant and reductant, respectively.
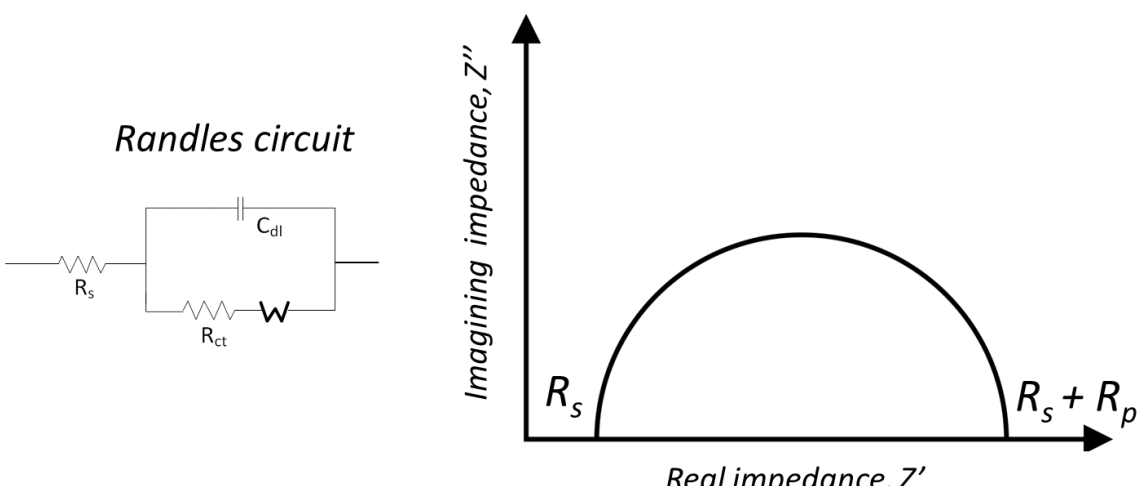

Figure 4.8 Schematic representation of Randles equivalent circuit (left) and Nyquist diagram for impedance spectroscopy. 


\section{SUMMARY OF PAPERS}

Eight papers in two different categories are presented. In the first section, the interfacing of novel materials for the application enzyme-based biosensors, are described in three different papers (Paper I, II, and III). The following section covers one review (Paper IV) and four different papers (Paper V, VI, VII and VIII), which are mainly related to switchable bioelectronics.

\subsection{Papers I-III}

\section{Paper I: Template-Directed Hierarchical Self-Assembly of Graphene Based Hybrid Structure for Electrochemical Biosensing}

Onur Parlak, Atul Tiwari, Anthony P. F. Turner, Ashutosh Tiwari

Biosensors and Bioelectronics, 2013, 49, 53

In this study, surface functionalised graphene is employed as a template material for directing the assembly of enzyme and nanoparticles as a bioelectrode for electrochemical enzyme-based sensing. The driving force to direct the assembly on the graphene was modification of the surface via the anionic surfactant, sodium dodecyl benzenesulfonate (SDBS). Following surface functionalisation, cholesterol oxidase and cholesterol esterase were assembled on the electrode together with gold nanoparticles.

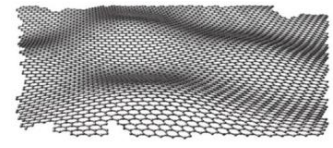

1) Surface Functionalisatio

2) Decoration with Au NPs

3) Enzyme immobilisation
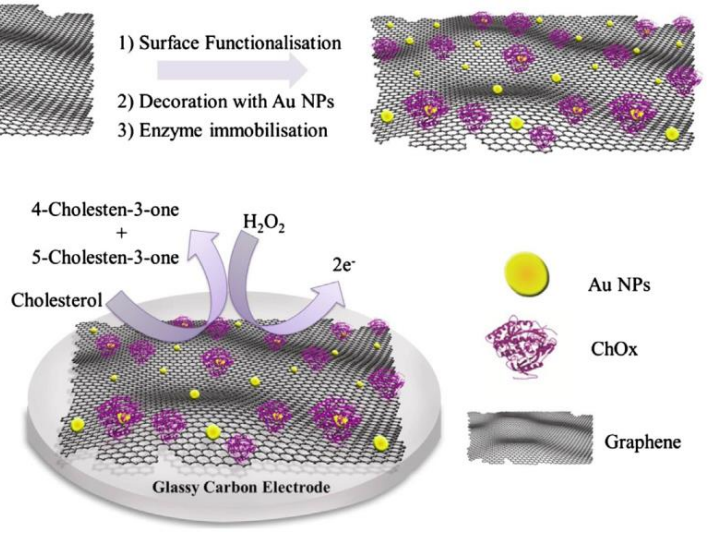

Schematics of surface modification of graphene and sensing process on the bioelectrode surface 
Here, hydrogen peroxide and cholesterol were used as two representative analytes in order to demonstrate the electrochemical sensing capacity of the designed bioelectrode. The results show that design of the system is highly sensitive $\left(3.14 \mathrm{~mA} / \mathrm{mM} / \mathrm{cm}^{2}\right)$ to cholesterol with $0.05 \mu \mathrm{M}$ detection limit in the range of 0.05-0.35 mM. More importantly, here we have shown that the templatedirected self-assembly approach can be an alternative way to generate hybrid biointerfaces with high enzyme loading capacity and with high stability. 
Paper II: Two-dimensional Gold-Tungsten Disulphide Bio-interface for High-throughput Electrocatalytic Nanobioreactors

Onur Parlak, Prethi Seshadri, Ingemar Lundström, Anthony P. F. Turner, Ashutosh Tiwari

Advanced Materials Interfaces, 2014, 1, 1400136

In this study, two-dimensional gold-nanoparticle decorated tungsten disulphides $\left(\mathrm{WS}_{2}\right)$ have been employed, for the first time, as a bio-interface element after conjugation with Horseradish peroxidase (HRP) for electrochemical hydrogen peroxide sensing $\left(\mathrm{H}_{2} \mathrm{O}_{2}\right)$. The 2D WS2 nanosheets have been used due to their promising physical and chemical properties, such as charge density, large surface area, high electron mobility and transport, together with tunable band gap and high density of electronic state.

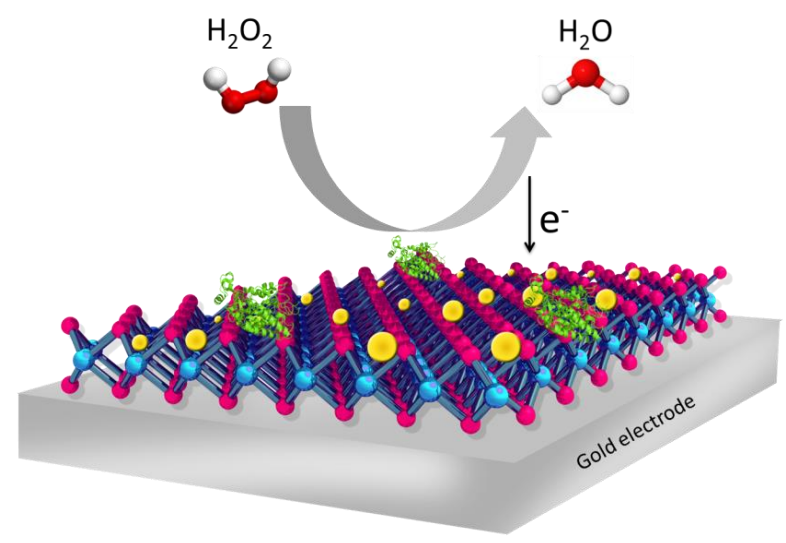

Schematic representation of $\mathrm{A} u \mathrm{NP} / W \mathrm{~W}_{2}$ interface for electrochemical $\mathrm{H}_{2} \mathrm{O}_{2}$ sensing

The bioelectrode had high sensitivity $\left(11.64 \mu \mathrm{A} / \mu \mathrm{M} / \mathrm{cm}^{2}\right)$ and a low detection limit $(0.085 \mu \mathrm{M})$ with linear detection range $(0.05$ to $12.0 \mathrm{mM})$. 


\section{Paper III: Probing Ultra-Lightweight Aerographite Properties for Efficient}

Bioelectrocatalysis

Onur Parlak, Yogendra K. Mishra, Anton Grigoriev, Matthias Mecklenburg, Karl Schulte, Rajeev Ahuja, Rainer Adelung, Anthony P. F. Turner, Ashutosh Tiwari (Manuscript)

A range of macro- and nano-scale carbon-based materials with different size and morphology can offer quite different catalytic and surface properties, andhave been commonly employed for the development of efficient interface materials in the area of electronic and energy applications. In this study, we have introduced, for the first time, an ultra-light weight aerographite material as a novel electrocatalytic interface and efficient bio-interface for enzyme-based biosensing. Here, we self-assembled Horseradish peroxidase with an aerographite structure to produce a catalytic interface for electrochemical detection of peroxide. The designed interfacedelivered high sensitivity $\left(16.9 \mu \mathrm{A} / \mu \mathrm{M} / \mathrm{cm}^{2}\right)$, a low detection limit $(0.054 \mu \mathrm{M})$ over a wide linear range $(0.01$ to $5.1 \mathrm{mM})$. We have also modelled and shown the origin of the catalytic activity and solution properties of the electrode material, together with the reaction mechanisms during enzymatic reactions using density functional theory. 


\subsection{Papers IV-V}

\section{Paper IV: Switchable Bioelectronics}

Onur Parlak, Anthony P. F. Turner

Biosensors and Bioelectronics, 2015 (In press) doi:10.1016/j.bios.2015.06.023

In this review article, we have focused on the emerging area of switchable bioelectronics by considering electrochemical applications of various bio-interfaces. The recent progress and early fundamental studies are highlighted and we have sought plausible explanations as to why switchable bioelectronics is important and how can we use the idea of switchable bioelectronics in real device applications. Here, we discuss physical stimuli, including light, temperature, potential and magnetically switchable surfaces and chemically stimulated interfaces by focusing on the latest efforts reported in the literature.

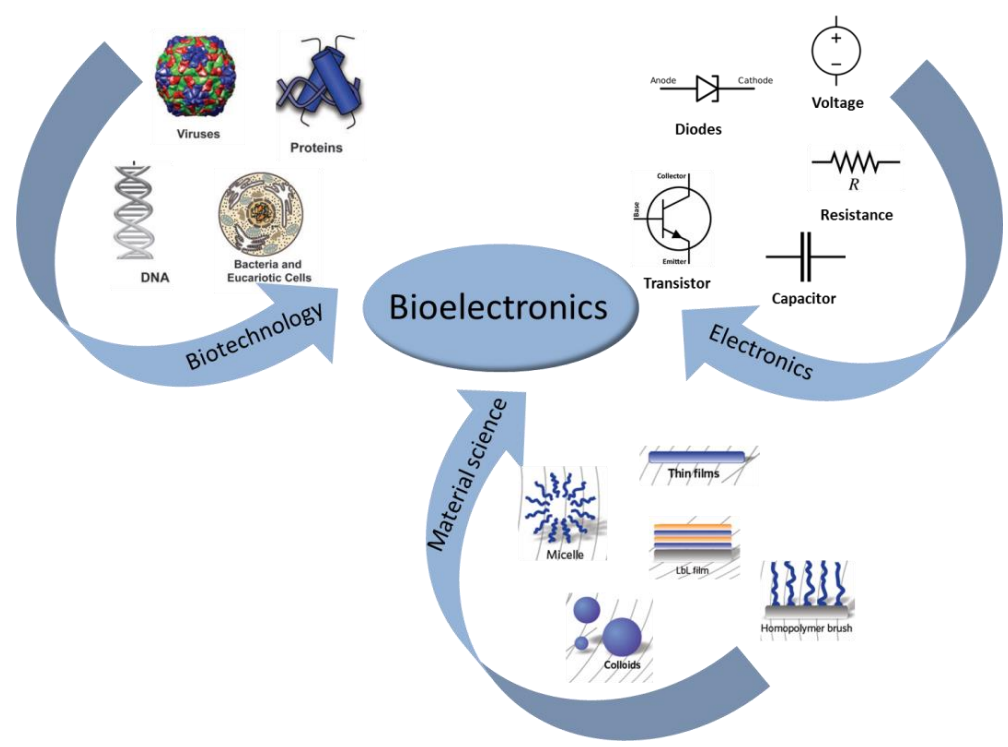

Illustrations of merging the area of biotechnology, electronics, and materials science 


\section{Paper V: On/Off-Switchable Zipper-Like Bioelectronics on a Graphene In- terface}

Onur Parlak, Anthony P. F. Turner, Ashutosh Tiwari

Advanced Materials, 2014, 26, 482

In this paper, we studied the fabrication of temperature switchable bio-interfaces using graphene-stimuli-responsive polymer hybrids to control and regulate enzyme-based biomolecular reactions. Following on from our earlier work (paper I), we elucidated an on-off switchable graphene-cholesterol oxidase-poly $(N$ isopropylacrylamide) polymer (PNIPAAM) based bio-interface that is capable of positively responding to external temperature change. At relatively low temperature conditions such as $20^{\circ} \mathrm{C}$, hydrogen bonds formed an interaction between surface modified graphene and polymer, which creates a coalescence of the graphene interface, thereby resulting in considerable shrinkage at the interface. The shrinking conditions restrict the access of enzyme to its substrate, resulting in a decrease in the diffusion of reactants and the consequent activity of the system. In contrast, under high temperature conditions such as at $40{ }^{\circ} \mathrm{C}$, hydrogen bonding is broken. The disappearance of hydrogen bond at the interface allows access of the biosubstrate to the enzyme, and in this way biocatalytic interaction is facilitated.

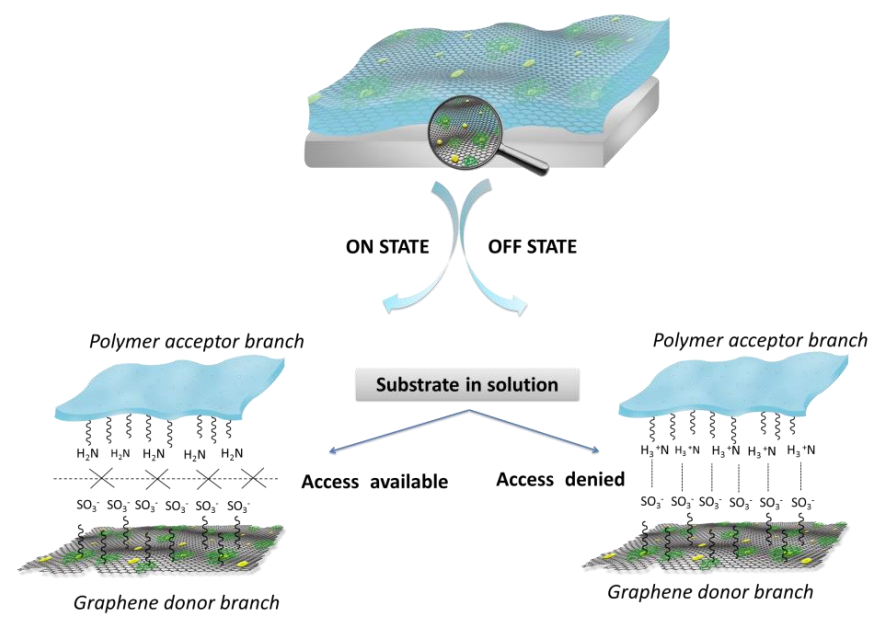

Representations of an on/off switchable bioelectrocatalytic graphene interface

More importantly, we have elaborated the morphological changes of polymer using in-situ scanning electrochemical microscopy. This study provides the first example of responsive bioelectronics being achieved on a two-dimensional graphene interface by controlling the external temperature as an on/offswitchable model. 


\section{Paper VI: pH-induced on/off-switchable graphene bioelectronics}

Onur Parlak, Anthony P. F. Turner, Ashutosh Tiwari

Journal of Materials Chemistry B, 2015 (Accepted)

Chemically-stimulated bio-interfaces comprise stimuli-responsive elements together with biomolecules, which are able to deliver functionally reversible reactivity with their corresponding analytes. This allows an event to positively respond to the activity of another biological event, such as an enzyme-based electrocatalytic reaction modulated via $\mathrm{pH}$ change of the medium. In this study, we demonstrated the development of a $\mathrm{pH}$-encoded bio-catalysis by employing $\mathrm{pH}$ responsive poly(4-vinyl pyridine, $\mathrm{P} 4 \mathrm{VP}$ ) graphene oxide bio-interfaces to control and regulate enzyme-based molecular interactions. Using electrochemical methods, we show that tunable interfacial electrochemical properties can be generated by relatively modest change in $\mathrm{pH}$ of the medium. The resulting switchable interface facilitated highly specific, on-demand operation of biosensors and biofuel cells, which has significant potential in a wide range of analytical and energy harvesting applications.
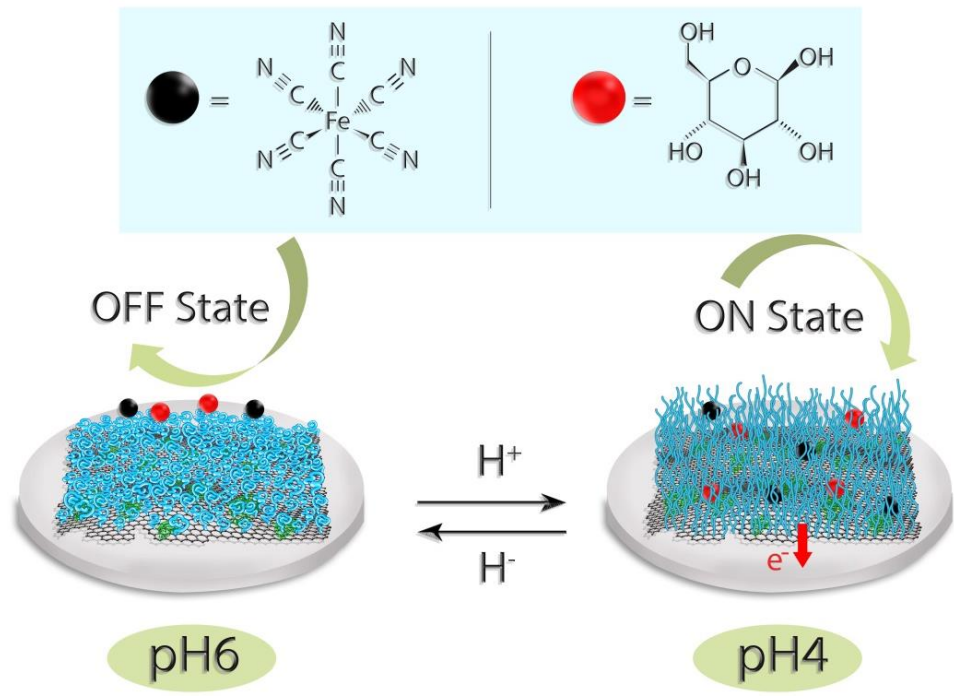

Representations of $p H$-encoded switchable graphene interfaces under two different $\mathrm{pH}$ conditions 


\section{Paper VII: Light-triggered on/off-switchable graphene-based bioelectronics}

Onur Parlak, Selim Beyazit, Mohammed J. Jafari, Bernadette Tse Sum Bui, Karsten Haupt, Ashutosh Tiwari, Anthony P. F Turner

\section{(Submitted)}

In this study, similar to the temperature and $\mathrm{pH}$ switchable interface, we designed a light switchable interface consisting of a light-responsive polymer with twodimensional graphene. The bio-interface consists of a pyrroloquinoline quinone (PQQ)-dependent glucose dehydrogenase, immobilised on two-dimensional graphene and polyacrylamide co-polymerised with spiropyran methacrylate (poly(Aam-co-SPMA), which is electrostatically assembled together.

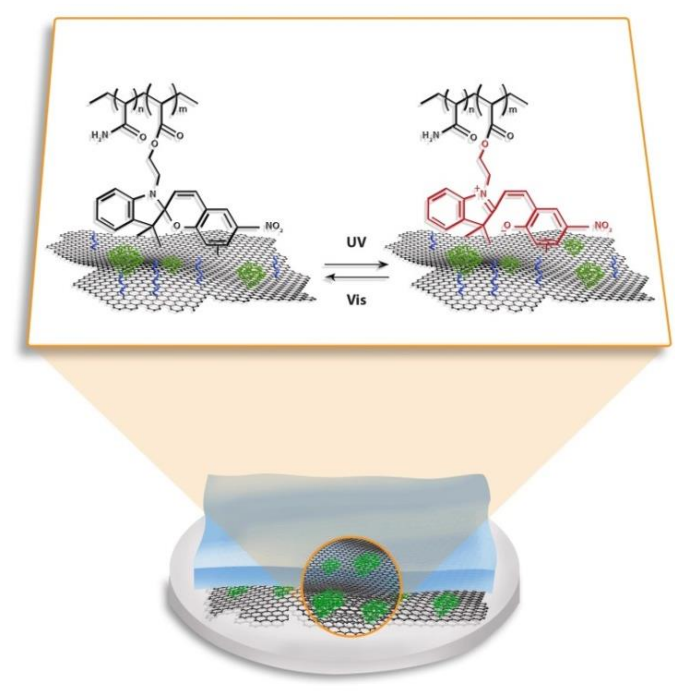

Schematic of the light switchable bioelectrocatalytic graphene interface

Light responsive poly(Aam-co-SPMA) is obtained by free radical copolymerisation of spiropyran methacrylate (SP) and acrylamide monomers. In the polymer chain, SP units are converted to charge-separated merocyanine (MC) upon exposure to UV light and return back to neutral SP under visible light or dark conditions. To show the light-sensitive character of the bio-interface, cyclic voltammetry, impedance spectroscopy, amperometry, charge-discharge tests, contact angle measurements and optical characterisation were performed by irradiating the system at different wavelengths of light to produce an "ON" state ( $\lambda$ $\leq 385 \mathrm{~nm}, \mathrm{UV}$ ) and an "OFF" state ( $\lambda \geq 420 \mathrm{~nm}$, daylight). 


\section{Paper VIII: Programmable bioelectronics in a stimuli -encoded 3D graphene}

Onur Parlak, Selim Beyazit, Bernadette Tse Sum Bui, Karsten Haupt, Anthony P. F Turner, Ashutosh Tiwari

\section{(Submitted)}

In this study, we introduce a physically controlled bio-interface which can be programmed using two different stimuli on one electrode. Here, we combined two concepts that are presented in Papers V and VII. By using the same methodology, we aim to go beyond the single switchable system, and we attempt to program and control via external physical stimuli. We create two different electrode designs that allow us to program interfacial electrochemical properties. The designed bio-electrodes comprise light and temperature responsive compartments in different orientations, which allow us to build Boolean logic gates (OR and AND) based on enzymatic interactions to deliver logic operations.

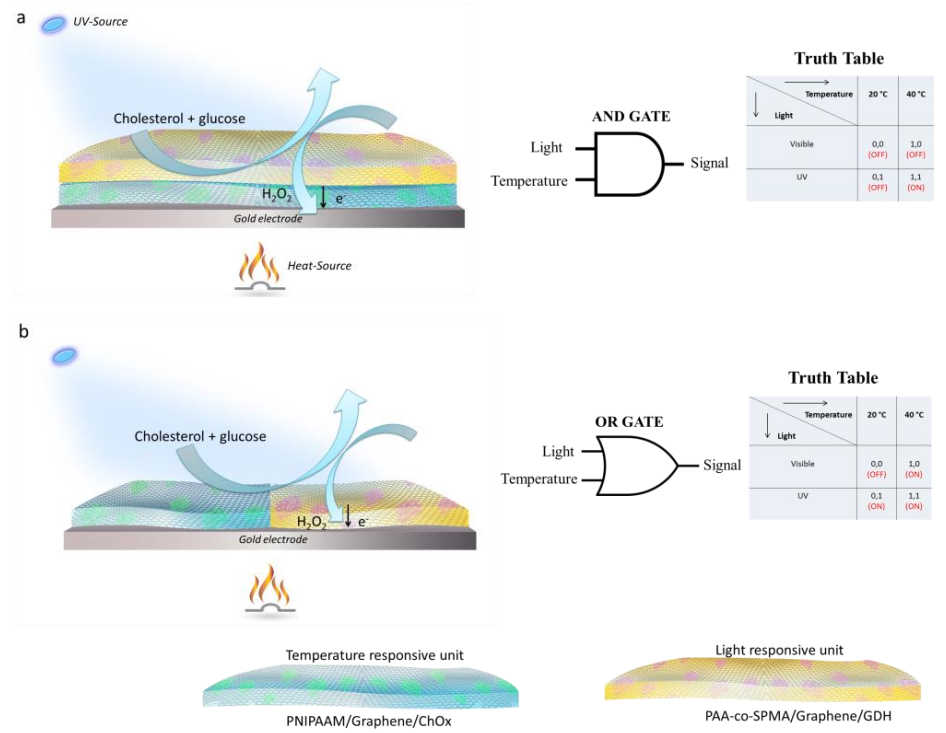

Logic gate designs for programmable bio-electrodes

These critical features of an externally controlled biocomputing system are a key requirement for further construction of more complex bio-molecular systems to segregate reaction conditions at a molecular level. It is worth noting that stimuli-encoded material as a trimming element could provide a way to regulate from the 'on-state' to an 'off-state' and/or to programme the rate of biological reactions via slow-to-medium-to-high and vice-versa. 


\section{FUTURE OUTLOOKS}

Interfacing nanomaterials for bioelectronic applications: A wide range of materials with different compositions, different morphologies, size and shape have been explored to improve electrical communication between biological and electronic components. All these novel materials and methods bring us closer to a better understanding of charge-transfer transport and electron-transfer pathways in biological systems. However, scaling-up the system and mass production for commercialisation still await solution for the interfacing nanomaterials in bioelectronic application.

Switchable bio-interfaces: It is always hard to predict the impact of emerging technologies and difficult to see where these technologies will evolve? But adding strong underpinning arguments can help the area of interest evolve to be a better platform which provides both practical and fundamental solutions for the society. Following from this motivation, various switchable bio-interfaces are developed in order to understand the relationship between enzyme-based catalytic systems and changing environmental conditions on the basis of electrochemical interface. It is clear that there is a long way to go before solving critical issues such as scaling up/down the switchable interfaces depending on the application and reversibility of the system.

Programmable bioelectronics: In the work leading up to this thesis the designing of "switchable bioelectronics" technology based on stimuli-responsive surfaces have evolved through a programmable bio-interface design which can help to segregate reaction conditions on electrode interface and can provide a way to programme the rate of biological reactions. However, this area is still its infancy and needed to be explored more.

If all these problems mentioned, are solved in near future, we may see, hopefully, new applications that will emerge based on the concept presented in this thesis. 


\section{REFERENCES}

(1) Parlak, O.; Turner, A. P. F. Biosensors \& Bioelectronics 2015, http://dx.doi.org/10.1016/j.bios.2015.06.023i

(2) Turner, A. P. F. Chemical Society Reviews 2013, 42, 3184.

(3) Du, J.; Catania, C.; Bazan, G. C. Chemistry of Materials 2014, 26, 686.

(4) Willner, I.; Katz, E. Bioelectronics: From Theory to Applications; Wiley, 2006.

(5) Turner, A. P. F.; Karube, I.; Wilson, G. S. Biosensors: fundamentals and applications; Oxford University Press, 1987.

(6) Turner, A. P. F. Science 2000, 290, 1315.

(7) Noy, A. Advanced Materials 2011, 23, 807.

(8) Windmiller, J. R.; Wang, J. Electroanalysis 2013, 25, 29.

(9) Kwak, Y. H.; Choi, D. S.; Kim, Y. N.; Kim, H.; Yoon, D. H.; Ahn, S.-S.; Yang, J.-W.; Yang, W. S.; Seo, S. Biosensors \& Bioelectronics 2012, 37, 82.

(10) Bae, S.; Kim, H.; Lee, Y.; Xu, X. F.; Park, J. S.; Zheng, Y.; Balakrishnan, J.; Lei, T.; Kim, H. R.; Song, Y. I.; Kim, Y. J.; Kim, K. S.; Ozyilmaz, B.; Ahn, J. H.; Hong, B. H.; Iijima, S. Nature Nanotechnology 2010, 5,574 .

(11) Eda, G.; Fanchini, G.; Chhowalla, M. Nature Nanotechnology 2008 ,

(12) Kim, K. S.; Zhao, Y.; Jang, H.; Lee, S. Y.; Kim, J. M.; Kim, K. S.; Ahn, J.-H.; Kim, P.; Choi, J.-Y.; Hong, B. H. Nature 2009, 457, 706.

(13) Davis, J. J.; Morgan, D. A.; Wrathmell, C. L.; Axford, D. N.; Zhao, J.; Wang, N. Journal of Materials Chemistry 2005, 15, 2160.

(14) Nel, A. E.; Maedler, L.; Velegol, D.; Xia, T.; Hoek, E. M. V.; Somasundaran, P.; Klaessig, F.; Castranova, V.; Thompson, M. Nature Materials $2009,8,543$.

(15) Callister, W. D.; Rethwisch, D. G. Fundamentals of Materials Science and Engineering: An Integrated Approach; Wiley, 2012.

(16) Fahlman, B. D. Materials Chemistry; Springer, 2011.

(17) Carter, G. F.; Carter, G. F.; Donald E. Paul, e. Materials Science and Engineering; A S M International, 1991.

(18) Rosoff, M. Nano-Surface Chemistry; CRC Press, 2001.

(19) Daniel, M. C.; Astruc, D. Chemical Reviews 2004, 104, 293.

(20) Ramsden, J. Nanotechnology: An Introduction; Elsevier Science, 2011 .

(21) Fahrner, W. Nanotechnology and Nanoelectronics: Materials, Devices, Measurement Techniques; Springer Berlin Heidelberg, 2005.

(22) Zhou, W.; Wang, Z. L. Scanning Microscopy for Nanotechnology: Techniques and Applications; Springer, 2007.

(23) Deshapriya, I. K.; Kumar, C. V. Langmuir 2013, 29, 14001. 1312 .

(24) Whitesides, G. M.; Mathias, J. P.; Seto, C. T. Science 1991, 254,

(25) Schmid, G. Nanotechnology: Principles and fundamentals; WileyVch, 2008. 
(26) Pöschl, V. An Introduction to the Chemistry of Colloids: A Compendium of Colloidal Chemistry for Students, Teachers and Works Managers; C. Griffin, limited, 1910.

(27) Pashley, R.; Karaman, M. Applied Colloid and Surface Chemistry; Wiley, 2005.

(28) Birdi, K. S. Handbook of Surface and Colloid Chemistry, Second Edition; CRC Press, 2002.

(29) Burda, C.; Chen, X. B.; Narayanan, R.; El-Sayed, M. A. Chemical Reviews 2005, 105, 1025.

(30) Russel, W. B.; Saville, D. A.; Schowalter, W. R. Colloidal Dispersions; Cambridge University Press, 1992.

2115 .

(31) Puntes, V. F.; Krishnan, K. M.; Alivisatos, A. P. Science 2001, 291,

(32) Bostrom, M.; Williams, D. R. M.; Ninham, B. W. Physical Review Letters 2001, 87.

1.

(33) Ninham, B. W. Advances in Colloid and Interface Science 1999, 83,

(34) Missana, T.; Adell, A. Journal of Colloid and Interface Science $\mathbf{2 0 0 0}, 230,150$.

(35) Hough, D. B.; White, L. R. Advances in Colloid and Interface Science 1980, 14, 3 .

(36) Bergstrom, L. Advances in Colloid and Interface Science 1997, 70,

(37) Bostrom, M.; Deniz, V.; Franks, G. V.; Ninham, B. W. Advances in Colloid and Interface Science 2006, 123, 5.

(38) Leong, Y. K.; Ong, B. C. Powder Technology 2003, 134, 249. 390.

(39) Napper, D. H. Journal of Colloid and Interface Science 1977, 58, 1979.

(40) Gimarc, B. M. Journal of the American Chemical Society 1983, 105,

(41) Hsu, M. F.; Dufresne, E. R.; Weitz, D. A. Langmuir 2005, 21, 4881.

(42) Vezenov, D. V.; Noy, A.; Rozsnyai, L. F.; Lieber, C. M. Journal of the American Chemical Society 1997, 119, 2006.

(43) Kim, J.; Cote, L. J.; Kim, F.; Yuan, W.; Shull, K. R.; Huang, J. X. Journal of the American Chemical Society 2010, 132, 8180.

(44) Hammond, P. T. Advanced Materials 2004, 16, 1271.

(45) Davis, J. A.; James, R. O.; Leckie, J. O. Journal of Colloid and Interface Science 1978, 63, 480. 131.

(46) Oldham, K. B. Journal of Electroanalytical Chemistry 2008, 613,

(47) Zukoski, C. F.; Saville, D. A. Journal of Colloid and Interface Science 1986, 114, 32.

(48) Levine, S.; Marriott, J. R.; Neale, G.; Epstein, N. Journal of Colloid and Interface Science 1975, 52, 136.

(49) Miyauchi, M.; Ikezawa, A.; Tobimatsu, H.; Irie, H.; Hashimoto, K. Physical Chemistry Chemical Physics 2004, 6, 865.

(50) Revil, A.; Pezard, P. A.; Glover, P. W. J. Journal of Geophysical Research-Solid Earth 1999, 104, 20021. 1978, 23, 533 .

(51) Blokhra, R. L.; Parmar, M. L.; Sharma, V. P. Electrochimica Acta

(52) Hjerten, S. Journal of Chromatography 1985, 347, 191. 
(53) Ohshima, H. Journal of Colloid and Interface Science 1996, 180, 65.

(54) Squires, T. M.; Bazant, M. Z. Journal of Fluid Mechanics 2006, 560, 217.

(55) Squires, T. M.; Bazant, M. Z. Journal of Fluid Mechanics 2004, 509,

(56) Zhou, Z. Y.; Tian, N.; Li, J. T.; Broadwell, I.; Sun, S. G. Chemical Society Reviews 2011, 40, 4167.

(57) O'Shea, S. J.; Welland, M. E. Langmuir 1998, 14, 4186.

(58) Tang, L. H.; Chang, H. X.; Liu, Y.; Li, J. H. Advanced Functional Materials 2012, 22, 3083 .

(59) Oliveira, O. N.; Iost, R. M.; Siqueira, J. R.; Crespilho, F. N.; Caseli, L. Acs Applied Materials \& Interfaces 2014, 6, 14745.

(60) Wang, Y.; Li, Z. H.; Wang, J.; Li, J. H.; Lin, Y. H. Trends in Biotechnology 2011, 29, 205.

(61) Polizu, S.; Savadogo, O.; Poulin, P.; Yahia, L. Journal of Nanoscience and Nanotechnology 2006, 6, 1883.

(62) Whitesides, G. M. Nature Biotechnology 2003, 21, 1161.

(63) Mertz, D.; Vogt, C.; Hemmerle, J.; Mutterer, J.; Ball, V.; Voegel, J. C.; Schaaf, P.; Lavalle, P. Nature Materials 2009, 8, 731.

(64) Chaudhuri, S. K.; Lovley, D. R. Nature Biotechnology 2003, 21,

(65) Shleev, S.; Tkac, J.; Christenson, A.; Ruzgas, T.; Yaropolov, A. I.; Whittaker, J. W.; Gorton, L. Biosensors \& Bioelectronics 2005, 20, 2517.

(66) Burroughes, J. H.; Bradley, D. D. C.; Brown, A. R.; Marks, R. N.; Mackay, K.; Friend, R. H.; Burn, P. L.; Holmes, A. B. Nature 1990, 347, 539.

(67) Rivnay, J.; Owens, R. M.; Malliaras, G. G. Chemistry of Materials 2014, 26, 679 . 3201 .

(68) Berggren, M.; Richter-Dahlfors, A. Advanced Materials 2007, 19,

(69) Friend, R. H.; Gymer, R. W.; Holmes, A. B.; Burroughes, J. H.; Marks, R. N.; Taliani, C.; Bradley, D. D. C.; Dos Santos, D. A.; Bredas, J. L.; Logdlund, M.; Salaneck, W. R. Nature 1999, 397, 121.

(70) Kulkarni, A. P.; Tonzola, C. J.; Babel, A.; Jenekhe, S. A. Chemistry of Materials 2004, 16, 4556.

(71) Kresse, G.; Hafner, J. Physical Review B 1994, 49, 14251.

(72) Kresse, G.; Furthmuller, J. Computational Materials Science 1996, 6,15 .

(73) Schmidt, M. W.; Baldridge, K. K.; Boatz, J. A.; Elbert, S. T.; Gordon, M. S.; Jensen, J. H.; Koseki, S.; Matsunaga, N.; Nguyen, K. A.; Su, S. J.; Windus, T. L.; Dupuis, M.; Montgomery, J. A. Journal of Computational Chemistry 1993, 14, 1347.

(74) Guenes, S.; Neugebauer, H.; Sariciftci, N. S. Chemical Reviews 2007, 107, 1324.

(75) Sirringhaus, H.; Brown, P. J.; Friend, R. H.; Nielsen, M. M.; Bechgaard, K.; Langeveld-Voss, B. M. W.; Spiering, A. J. H.; Janssen, R. A. J.; Meijer, E. W.; Herwig, P.; de Leeuw, D. M. Nature 1999, 401, 685.

(76) Duan, X. J.; Lieber, C. M. Nano Research 2015, 8, 1.

(77) Kleijn, S. E. F.; Lai, S. C. S.; Koper, M. T. M.; Unwin, P. R. Angewandte Chemie-International Edition 2014, 53, 3558. 155 .

(78) Li, Z.; Hu, N. F. Journal of Electroanalytical Chemistry 2003, 558, 
(79) Huang, X.-J.; O'Mahony, A. M.; Compton, R. G. Small 2009, 5, 776.

(80) Shan, D.; Wang, S.; Xue, H.; Cosnier, S. Electrochemistry Communications 2007, 9, 529.

(81) Xiao, Y.; Patolsky, F.; Katz, E.; Hainfeld, J. F.; Willner, I. Science 2003, 299, 1877.

(82) Haram, S. K.; Quinn, B. M.; Bard, A. J. Journal of the American Chemical Society 2001, 123,8860.

(83) Parlak, O.; Seshadri, P.; Lundstrom, I.; Turner, A. P. F.; Tiwari, A. Advanced Materials Interfaces 2014, 1 .

(84) Coleman, J. N.; Lotya, M.; O'Neill, A.; Bergin, S. D.; King, P. J.; Khan, U.; Young, K.; Gaucher, A.; De, S.; Smith, R. J.; Shvets, I. V.; Arora, S. K.; Stanton, G.; Kim, H.-Y.; Lee, K.; Kim, G. T.; Duesberg, G. S.; Hallam, T.; Boland, J. J.; Wang, J. J.; Donegan, J. F.; Grunlan, J. C.; Moriarty, G.; Shmeliov, A.; Nicholls, R. J.; Perkins, J. M.; Grieveson, E. M.; Theuwissen, K.; McComb, D. W.; Nellist, P. D.; Nicolosi, V. Science 2011, 331, 568.

(85) Akinwande, D.; Petrone, N.; Hone, J. Nature Communications 2014, 5 .

(86) Ambrosi, A.; Chua, C. K.; Bonanni, A.; Pumera, M. Chemical Reviews 2014, 114, 7150.

(87) Dagotto, E.; Rice, T. M. Science 1996, 271, 618.

(88) Brownson, D. A. C.; Kampouris, D. K.; Banks, C. E. Chemical Society Reviews 2012, 41, 6944.

(89) Brownson, D. A. C.; Banks, C. E. Analyst 2010, 135, 2768.

(90) Wick, P.; Louw-Gaume, A. E.; Kucki, M.; Krug, H. F.; Kostarelos, K.; Fadeel, B.; Dawson, K. A.; Salvati, A.; Vazquez, E.; Ballerini, L.; Tretiach, M.; Benfenati, F.; Flahaut, E.; Gauthier, L.; Prato, M.; Bianco, A. Angewandte Chemie-International Edition 2014, 53, 7714.

(91) Parlak, O.; Turner, A. P. F.; Tiwari, A. Advanced Materials 2014, 26,482 .

(92) Parlak, O.; Tiwari, A.; Turner, A. P. F.; Tiwari, A. Biosensors \& Bioelectronics 2013, 49, 53.

(93) Li, W.; Tan, C.; Lowe, M. A.; Abruna, H. D.; Ralph, D. C. Acs Nano 2011, 5, 2264.

(94) Wang, Y.; Tang, L. Biosensors \& Bioelectronics 2015, 67, 18.

(95) Nature Materials 2014, 13, 1073.

(96) Chhowalla, M.; Shin, H. S.; Eda, G.; Li, L.-J.; Loh, K. P.; Zhang, H. Nature Chemistry 2013, 5, 263. 3766.

(97) Xu, M.; Liang, T.; Shi, M.; Chen, H. Chemical Reviews 2013, 113,

(98) Russell, T. P. Science 2002, 297, 964.

(99) Lahann, J.; Mitragotri, S.; Tran, T. N.; Kaido, H.; Sundaram, J.; Choi, I. S.; Hoffer, S.; Somorjai, G. A.; Langer, R. Science 2003, 299, 371.

(100) Sun, A.; Lahann, J. Soft Matter 2009, 5, 1555.

(101) Mendes, P. M. Chemical Society Reviews 2008, 37, 2512.

(102) Stuart, M. A. C.; Huck, W. T. S.; Genzer, J.; Muller, M.; Ober, C.; Stamm, M.; Sukhorukov, G. B.; Szleifer, I.; Tsukruk, V. V.; Urban, M.; Winnik, F.; Zauscher, S.; Luzinov, I.; Minko, S. Nature Materials 2010, 9, 101.

(103) Alarcon, C. D. H.; Pennadam, S.; Alexander, C. Chemical Society Reviews 2005, 34, 276.

(104) Ahn, S.-K.; Kasi, R. M.; Kim, S.-C.; Sharma, N.; Zhou, Y. Soft Matter 2008, 4, 1151. 
1265 .

(105) Jones, D. M.; Brown, A. A.; Huck, W. T. S. Langmuir 2002, 18 ,

(106) Kwok, D. Y.; Neumann, A. W. Advances in Colloid and Interface Science 1999, 81, 167.

(107) Browne, W. R.; Feringa, B. L. In Annual Review of Physical Chemistry 2009; Vol. 60, p 407.

(108) Klajn, R. Chemical Society Reviews 2014, 43, 148.

(109) Bocharova, V.; Katz, E. Chemical Record 2012, 12, 114.

(110) Lutz, J. F. Advanced Materials 2011, 23, 2237.

(111) Pranzetti, A.; Mieszkin, S.; Iqbal, P.; Rawson, F. J.; Callow, M. E.; Callow, J. A.; Koelsch, P.; Preece, J. A.; Mendes, P. M. Advanced Materials 2013, 25, 2181.

(112) Katz, E.; Minko, S.; Halamek, J.; MacVittie, K.; Yancey, K. Analytical and Bioanalytical Chemistry 2013, 405, 3659.

(113) Katz, E. Electroanalysis 2010, 22, 744.

(114) Privman, M.; Tam, T. K.; Pita, M.; Katz, E. Journal of the American Chemical Society 2009, 131, 1314.

(115) Contin, A.; Plumeré, N.; Schuhmann, W. Electrochemistry Communications 2015, 51, 50.

(116) Tam, T. K.; Zhou, J.; Pita, M.; Ornatska, M.; Minko, S.; Katz, E. Journal of the American Chemical Society 2008, 130, 10888.

(117) Tam, T. K.; Ornatska, M.; Pita, M.; Minko, S.; Katz, E. Journal of Physical Chemistry C 2008, 112, 8438.

(118) Strack, G.; Ornatska, M.; Pita, M.; Katz, E. Journal of the American Chemical Society 2008, 130, 4234.

(119) Wang, L.; Lian, W.; Yao, H.; Liu, H. Acs Applied Materials \& Interfaces 2015, 7, 5168.

(120) Katz, E.; Privman, V. Chemical Society Reviews 2010, 39, 1835.

(121) Ikeda, M.; Tanida, T.; Yoshii, T.; Kurotani, K.; Onogi, S.; Urayama, K.; Hamachi, I. Nature Chemistry 2014, 6, 511.

(122) Matyjaszewski, K.; Davis, T. P. Handbook of Radical Polymerization; Wiley, 2002.

(123) Brazel, C. S.; Rosen, S. L. Fundamental Principles of Polymeric Materials; Wiley, 2012.

(124) Bevington, J. C. Radical polymerization; Academic Press, 1961.

(125) Moad, G.; Solomon, D. H. The Chemistry of Radical Polymerization; Elsevier, 2006.

(126) Seavey, K.; Liu, Y. A. Step-Growth Polymerization Process Modeling and Product Design; Wiley, 2009. 2000.

(127) Calleja, F. B.; Roslaniec, Z. Block Copolymers; Taylor \& Francis,

(128) Guisan, J. M. Immobilization of Enzymes and Cells; Humana Press, 2006.

(129) Mateo, C.; Palomo, J. M.; Fernandez-Lorente, G.; Guisan, J. M.; Fernandez-Lafuente, R. Enzyme and Microbial Technology 2007, 40, 1451.

(130) Drobnik, J.; Saudek, V.; Svec, F.; Kalal, J.; Barta, M.; Vojtisek, V. Biotechnology and Bioengineering 1979, 21, 1317.

(131) Fernandez-Pacheco, R.; Arruebo, M.; Marquina, C.; Ibarra, R.; Arbiol, J.; Santamaria, J. Nanotechnology 2006, 17, 1188.

(132) Scouten, W. H.; Luong, J. H. T.; Brown, R. S. Trends in Biotechnology 1995, 13, 178. 
(133) Kuo, J. Electron Microscopy: Methods and Protocols; Humana Press, 2007.

(134) Goodhew, P. J.; Humphreys, J.; Beanland, R. Electron Microscopy and Analysis, Third Edition; Taylor \& Francis, 2000.

(135) Williams, D. B.; Carter, C. B. Transmission Electron Microscopy: A Textbook for Materials Science; Springer, 2009.

(136) Wells, O. C. Scanning electron microscopy; McGraw-Hill, 1974.

(137) Bard, A. J.; Faulkner, L. R. Electrochemical Methods: Fundamentals and Applications; Wiley, 2000.

(138) Compton, R. G.; Banks, C. E. Understanding Voltammetry; World Scientific, 2007.

(139) Jay, C. R. G. W.; Wadhawan, J. D.; Compton, R. G. Electrochemistry; Royal Society of Chemistry, 2013. 
PUBLICATIONS 



\section{Publications}

The articles associated with this thesis have been removed for copyright reasons. For more details about these see:

http://urn.kb.se/resolve?urn=urn:nbn:se:liu:diva-120990 